\title{
Vortex induced vibrations of a pivoted finite height cylinder at low Reynolds number
}

J.C. Cajas, ${ }^{1, \text { a) }}$ D. Pastrana, ${ }^{2}$ I. Rodríguez, ${ }^{3}$ O. Lehmkuhl, ${ }^{2}$ G. Houzeaux, ${ }^{2}$ M. Vázquez, ${ }^{2}$ and C. Treviño ${ }^{1,4}$

1) ENES - Unidad Mérida, Universidad Nacional Autónoma de México, Tablaje Catastral No. 6998, Carretera Mérida-Tetiz Km. 4.5, 97357 Ucú, Yucatán, México.

2) Barcelona Supercomputing Center (BSC), Nexus II Building c/Jordi Girona 29, 08034 Barcelona, Spain.

3) Turbulence and Aerodynamics Research Group (TUAREG). Universitat

Politècnica de Catalunya, Colom 11, 08222 Terrassa, Barcelona, Spain.

4) UMDI, Facultad de Ciencias, Universidad Nacional Autónoma de México, Puerto de Abrigo s/n, 97356 Sisal, Yucatán, México.

(Dated: 13 May 2021)

The vortex induced vibrations (VIV) of a pivoted cylinder with finite height have been numerically investigated. A mathematical model is introduced, described and the resulting equations numerically solved for low Reynolds number $R e=100,200$ and several combinations of the governing parameters. Results on the solid body trajectories, the maximum amplitude of the oscillations, the hydrodynamic force coefficients, the wake structure and details on the vortex shedding near the cylinder are presented and discussed. The numerical results compare reasonably well with the canonical system of VIV of two-degrees of freedom circular cylinder in the laminar regime. Also, qualitative similarities with closely related VIV systems at larger Re suggest interesting lines of future research. Analytical approximations for limiting cases are done and an excellent agreement with the numerical results is obtained.

a)Electronic mail: carlos.cajas@enesmerida.unam.mx 


\section{INTRODUCTION}

Vortex induced vibrations (VIV) of bluff bodies are present and have a determinant role in many physical problems. Making evident their relevance in applications such as marine devices and energy harvesting systems. The principal advances in the understanding of the fundamental physical characteristics of such systems have been achieved through the study of canonical problems of flow around elastically mounted or flexible cylinders. Enlightening reviews to understand the development of the theory and techniques used in the research of this phenomenon can be found in the work of Sarpkaya ${ }^{1}$, Williamson ${ }^{2}$ and Bearman ${ }^{3}$. As a first main point, it has been shown that there exists a region in the parameter space, commonly known as the lock-in range, where large amplitude oscillations are obtained. In the lock-in range the natural frequency of the cylinder is close to the vortex-shedding frequency. Thus, synchronization is obtained and considerable oscillations appear. The relevant non-dimensional parameter of this kind of systems used to characterize the lock-in range is the reduced velocity, $u_{r}^{*}=u_{0} / f_{n} d$, which is the inverse of the natural frequency of the system scaled by the factor $u_{0} / d$, where $u_{0}$ is a representative velocity of the flow and $d$ is the diameter of the cylinder.

Depending on the type of system (i.e., elastically mounted freely vibrating cylinders with one-degree (1dof), or two-degrees of freedom (2dof), or pivoted or cantilevered cylinders), as well as on the values of the reduced velocity, the damping of the system and the mass ratio between the cylinder and the displaced mass of fluid, different branches of response in the oscillations might appear. In 1dof systems (see for instance Williamson ${ }^{2}$ and $\mathrm{Khalak}^{4}$ ), three branches of response have been identified: (i) the initial branch $(I)$, where the lock-in range begins and noticeable oscillations start to appear; the upper branch $(U)$, typical of low mass ratio systems and where large amplitude oscillations of about $1 d$ are obtained; the lower branch $(L)$, close to the end of the lock-in region where the amplitude of the oscillations diminishes before desynchronization is obtained. In 2dof systems, in addition to the branches observed in 1dof cylinders, for low-mass ratio systems an additional branch, the super-upper $(S U)$ has been reported to occur ${ }^{5}$. This branch is characterized by massive amplitude oscillations of about $1.5 \mathrm{~d}$. Each of these branches is also characterized by different shedding patterns: the $2 \mathrm{~S}$ mode, two single vortices shed per oscillation cycle occurs in the initial branch; the $2 \mathrm{P}$ mode, two vortex pairs shed per oscillation cycle, typical of the upper 


\begin{tabular}{|c|c|c|c|}
\hline \multicolumn{4}{|c|}{ Nomenclature } \\
\hline \multicolumn{2}{|c|}{ Mathematical model } & \multicolumn{2}{|l|}{ Nondimensional variables } \\
\hline$d$ & Cylinder diameter & $t^{*}=u_{0} t / d$ & Time \\
\hline$l$ & Cylinder length & $\boldsymbol{x}^{*}=\boldsymbol{x} / d$ & Space coordinates \\
\hline$A=d l$ & Reference surface & $m_{r}^{*}=\frac{I /(d h)}{\pi(d / 2)^{2} l \rho_{f}}$ & Reduced mass \\
\hline$\rho_{f}$ & Fluid density & $m_{g r}^{*}=\frac{u_{0}^{2} I}{m g d^{2} h}$ & Gravitational influence parameter \\
\hline$\mu_{f}$ & Fluid dynamic viscosity & $u_{r}^{*}=\frac{2 \pi u_{0}}{\sqrt{k /\left(I / h^{2}\right)} d}$ & Reduced velocity \\
\hline $\mathbf{u}_{f}$ & Fluid velocity & $\zeta_{i}^{*}=\frac{\alpha_{i}}{2 \sqrt{k I / h^{2}}}$ & Damping parameter \\
\hline $\mathbf{u}_{m}$ & Domain velocity (ALE) & $R e=\rho_{f} u_{o} d / \mu_{f}$ & Reynolds number \\
\hline $\mathbf{d}_{m}$ & Domain displacement (ALE) & & \\
\hline$c_{m}$ & Diffusion coefficient (ALE) & & \\
\hline$p$ & Pressure & & \\
\hline$x_{j}$ & Space coordinates & \multicolumn{2}{|l|}{ Analytical approximation } \\
\hline$t$ & Time & $\varepsilon$ & Expansion parameter \\
\hline$u_{0}$ & Inflow velocity & $\Theta, \Phi$ & Angular expansion variables \\
\hline$L$ & Lagrangian function & $S=\frac{2}{\omega_{0}^{2} \pi m_{r}^{*}}$ & Stiffness parameter \\
\hline$T, V$ & Kinetic, potential energy & $S_{g}=\frac{u_{r}^{* 2}}{(2 \pi)^{2} m_{g r}^{*}}$ & Weight parameter \\
\hline$\theta, \phi$ & Angular variables for the cylinder & $\beta_{x}=\frac{4 \pi \zeta_{\theta}^{*}}{u_{r}^{*}}, \beta_{y}=\frac{4 \pi \zeta_{\phi}^{*}}{\left(u_{r}^{*} \sin ^{2} \bar{\theta}\right)}$ & Damping parameters \\
\hline$h$ & Dist. cylinder center of mass - pivot & $\omega_{x}, \omega_{y}$ & Forcing frequencies \\
\hline$I$ & Inertia tensor & $\omega_{0}=2 \pi / u_{r}^{*}$ & Nondimensional natural frequency \\
\hline$k$ & Spring stiffness & $\sigma$ & Phase shift (force - displacement) \\
\hline$g$ & Gravitational acceleration & & \\
\hline $\mathbf{F}=\left(F_{x}, F_{y}, F_{z}\right)$ & Resultant fluid force on cylinder & & \\
\hline$Q_{j}, q_{j}$ & Generalized forces and coordinates & & \\
\hline$D_{j}, \alpha_{j}$ & Damping forces, dissipation constants & & \\
\hline$C_{j}$ & Force coefficients & & \\
\hline
\end{tabular}

and lower branches; and the $2 \mathrm{~T}$ mode, with two vortex triads shed per oscillation cycle which occurs in the super-upper branch. In the particular case of pivoted cylinders, the $2 \mathrm{C}$ mode related to vortex dislocations (a vortex division process due to presence of cells of different vortex-shedding modes along the span of the cylinder ${ }^{6-8}$ ) where two pair of corotating vortices are shed per oscillation cycle has also been reported ${ }^{7,9}$. A very complete description of the relations of the branch responses and the vortex shedding patterns for 2 dof elastically mounted cylinders can be found in the recent work of Pastrana ${ }^{10}$.

Through the years, numerical simulations addressing this kind of systems have been performed, trying to capture all the physical features of the flow and facing, at the same time, all the challenges common to all fluid-structure interaction (FSI) problems (two complete 
overviews of the state of the art for general FSI problems can be found in the work published by Bazilevs ${ }^{11}$ and Richter ${ }^{12}$ ). In the laminar regime, great advances have been obtained for elastically mounted cylinder systems, see for instance ${ }^{13-18}$. Very recently the VIV of elastically mounted rotating cylinders has been investigated ${ }^{19,20}$, and an analytical approach to obtain a reduced order model for VIV and galloping of square section cylinders has been performed $^{21}$. Meanwhile, in the turbulent regime, different approaches and models have been used to simulate the turbulent FSI and improve the reliability and accuracy of the results. Two outstanding examples follow. In Gsell et al. ${ }^{22}$, a 2 dof elastically mounted circular cylinder at $R e=3900$ was considered. The three branches of response were well reproduced by the simulation but the maximum amplitude of oscillations were underestimated in the SU branch and the $2 \mathrm{~T}$ vortex shedding pattern was not captured by the numerical solution. In Pastrana et. al. ${ }^{10}$, also a 2 dof elastically mounted circular cylinder was considered with $R e=$ 3900, 5300, 11000. The three response branches were reproduced; very accurate amplitudes were obtained in the simulation and the $2 \mathrm{~T}$ mode was observed.

At the present point, 1dof and 2dof elastically mounted cylinders have been the main systems of investigation, and the most relevant information on VIV has been obtained through the study of these systems. However, in practical cases, it is very often to encounter structures that oscillate with a non-uniform amplitude. Such systems have received relatively little attention and its potential for practical applications in marine structures and energy harvesting is a good motivation to encourage their study. Fujarra et al. ${ }^{23}$ conducted experiments on a cantilever cylinder with a low mass ratio over the range of Reynolds numbers of 1000-2500. They observed similarities with the 1dof rigid cylinder, but only the initial and lower branches were observed. At the same time, they conjectured the possibility that $2 \mathrm{P}$ and $2 \mathrm{~S}$ modes of vortex-shedding might co-exist along the span for amplitudes larger than 0.6. Later, in Flemming \& Williamson ${ }^{7}$ a pivoted cylinder was experimentally studied with different inertia ratios at Reynolds number around 1000. For the cases with moderate inertia-damping ratios, two amplitude response branches were observed, whereas for low inertia-damping ratios, three branches of response of the system were obtained. In their work, the maximum amplitudes for cylinders of different materials were reported, the $2 \mathrm{C}$ vortex-shedding pattern was characterized and its relation with vortex dislocations was pointed out. Also, a recent experiment on the VIV of a pivoted circular cylinder undergoing elliptic trajectories was conducted by Marble et al. ${ }^{9}$ for two different reduced velocities. The 
structure of the wake in the span-wise direction was examined using PIV measurements. Their results provide a quantitative assessment of the wake topology for this type of VIV. Two very interesting features of the pivoted cylinder shown in both studies are that vortex dislocations are present (as in the case of the fixed cantilevered cylinder ${ }^{6,24,25}$ ) and that different vortex-shedding patterns are obtained along the span of the cylinder. The numerical investigation of such systems have also received relatively little attention, examples of numerical investigations on flexible cantilevered cylinders have been published ${ }^{26,27}$ and to the best authors' knowledge no relevant numerical results for the case of pivoted cylinders have been published at the moment of writing this introduction.

The literature survey reflects that a great effort has been devoted to the study of the VIV of elastically mounted cylinders where the same amplitude of oscillation is present along all the span. Notably, numerical works have addressed almost exclusively this configuration with great success. Nonetheless, in many practical cases fixed points exist and give place to non-uniform amplitudes of oscillation along the span of the cylinder. This gives place to different vortex-shedding patterns and dynamical properties of the system. This kind of configuration has received relatively little attention from the numerical community. The objective of the present contribution is to introduce a mathematical model for this kind of systems and use it to numerically study the VIV of a pivoted cylinder of finite height at low Reynolds number.

The proposed system is depicted in Figure 1 and is based on previous numerical simulations developed by the authors to study a new design of wind energy generator ${ }^{28}$. The present work considers a simplification of the model of the energy harvesting device, and considers a laminar flow. This way, fundamental physical properties of this FSI phenomenon can be analyzed without adding the complexity of turbulence. For the parametric study, two Reynolds numbers are considered, $R e=100,200$. The influence of the reduced velocity and mass ratio, as well as the influence of the Reynolds number on the amplitude in the $\mathrm{U}$ branch are considered. Results on the maximum average amplitudes, the trajectories followed by the cylinder, the wake structures and details on the vortex shedding near the cylinder surface are provided. The organization of the paper is as follows: section II presents the mathematical model used to represent the physical system under consideration. Section III presents the numerical formulation employed to solve the governing equations. Section IV presents the numerical results obtained. Section V presents analytical approximations 

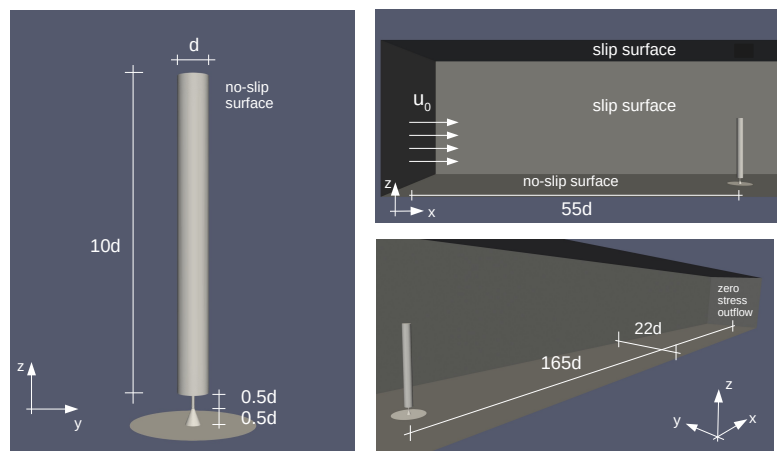

FIG. 1: Sketch of the system under study. (left) Close view of the cylinder and the pivot location. (right top) Side view and boundary conditions. (right bottom) Domain extension in the back of the cylinder.

for the solutions of the solid body equations. Finally, the conclusions and future work lines can be found in Section VI.

\section{PROBLEM STATEMENT}

The system under study is shown in Figure 1. It consists of a rigid mast of large aspect ratio (diameter/length) $A R=d / l=10$ attached to a torsion spring through a small bar of length $0.5 d$ connected to a fixed base. The mast is embedded in a Newtonian viscous fluid that flows with constant velocity $u_{0}$ far upstream of the mast. The fluid is considered to be incompressible and to have constant physical properties. The fluid domain represents a wind tunnel of $22 \mathrm{~d}$ in the cross-flow direction and $220 \mathrm{~d}$ in the streamwise direction. The mast is located at 55d from the wind tunnel entrance. Two Reynolds numbers are here considered, $R e=\rho_{f} u_{o} d / \mu_{f}=100,200$, here $\rho_{f}$ is the fluid density and $\mu_{f}$ the fluid viscosity. 


\section{A. Fluid component}

The fluid is modeled as an incompressible Newtonian fluid. In order to account for the movement of the solid body, the Navier-Stokes equations written in the Arbitrary Lagrangian Eulerian (ALE) formulation is used. In this framework, the mass and momentum conservation laws are written in a moving Eulerian domain, and the governing equations are then derived. The ALE formulation has been extensively used in FSI problems with great success $^{29-32}$. The authors have employed this formulation satisfactorily in ${ }^{28}$, where details of the implementation can be found. The resulting equations for a Newtonian viscous fluid are

$$
\begin{gathered}
\rho_{f} \frac{\partial \boldsymbol{u}_{f}}{\partial t}+\rho_{f}\left[\left(\boldsymbol{u}_{f}-\boldsymbol{u}_{m}\right) \cdot \nabla\right] \boldsymbol{u}_{f}-\mu_{f} \nabla^{2} \boldsymbol{u}_{f}+\nabla p=\rho_{f} \boldsymbol{f} \\
\nabla \cdot \boldsymbol{u}_{f}=0 .
\end{gathered}
$$

Here $\boldsymbol{u}_{f}$ is the flow velocity, $p$ is the pressure, $t$ is the time, $\boldsymbol{u}_{m}$ represents the domain velocity and $\boldsymbol{f}$ represents the body force. The domain velocity $\boldsymbol{u}_{m}$ is obtained from the domain displacement, $\boldsymbol{d}_{m}$, computed as the solution of the diffusion equation

$$
\nabla \cdot\left[c_{m} \nabla \boldsymbol{d}_{m}\right]=0
$$

with $c_{m}$ a diffusion coefficient used to control the deformation rates allowed for the elements depending on their aspect ratio and volume.

\section{B. Solid component}

For the solid component of the coupled problem, the motion of the mast can be described as a top that is not allowed to spin over its symmetry axis and is only allowed to deviate from the vertical axis in two directions following a Hookean law in the angular displacements. A very convenient reference system to describe this motion is the one shown in Figure 2, where an inertial system of reference is shown in lowercase letters and a moving reference frame attached to the rigid body is shown in uppercase letters, where $x$ is the direction of the main flow and $z$ is the vertical direction opposite to the gravitational acceleration.

The Lagrangian function of such system can be obtained in a very similar way as the one 


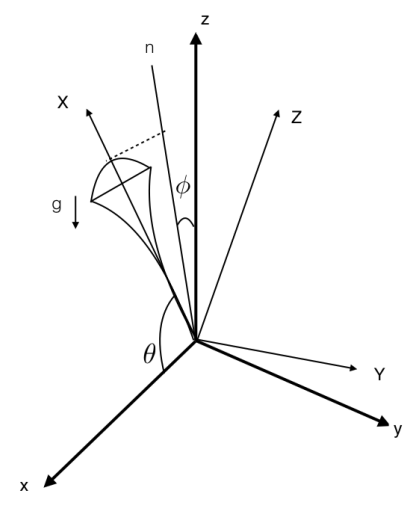

FIG. 2: Rigid body reference systems. Lowercase letters correspond to the inertial reference system and uppercase letters to the moving reference frame.

of the classical symmetric top, resulting

$$
L=T-V=\frac{1}{2}\left[I\left(\dot{\theta}^{2}+\dot{\phi}^{2} \sin ^{2} \theta\right)\right]+m g h \sin \theta \cos \phi-\frac{k h^{2}}{2}\left(1-\sin ^{2} \theta \cos ^{2} \phi\right),
$$

where the dot superscript denotes time derivatives in the usual way, $T$ and $V$ are the kinetic and the potential energies of the system, respectively. $\theta$ is measured from the $x$ axis and $\phi$ is the angle between the projection of the symmetry axis of the rigid body to the $y z$ plane and the $y$ axis. $I$ is the inertia tensor and $h$ is the distance between the center of mass of the body and the fixed point, $k$ is the spring stiffness and $g$ the gravitational acceleration. The resulting equations of motion are:

$$
\begin{array}{r}
I \ddot{\theta}-I \dot{\phi}^{2} \sin \theta \cos \theta+m g h \cos \theta \cos \phi-k h^{2}\left(\sin \theta \cos \theta \cos ^{2} \phi\right)=0, \\
I \sin ^{2} \theta \ddot{\phi}+2 I \sin \theta \cos \theta \dot{\theta} \dot{\phi}-m g h \sin \theta \sin \phi+k h^{2}\left(\sin \phi \cos \phi \sin ^{2} \theta\right)=0
\end{array}
$$

Equations 4 are valid for the cases where the only external forces are gravity and the resistance in the fixed point of the body. In the case of the FSI problem, there is a force coming from the interaction with the fluid that can not be written as the derivative of a known potential, here denoted as $\mathbf{F}=\left(F_{x}, F_{y}, F_{z}\right)$, its components are known in the inertial 
system of reference. In this case, the equations of motion are derived from the general expression:

$$
\frac{d}{d t}\left(\frac{\partial T}{\partial \dot{q}^{j}}\right)-\frac{\partial T}{\partial q^{j}}=Q_{j}
$$

where $Q_{j}$ are the generalized forces. In our case, the generalized forces can be separated in those that can be written as the derivatives of a potential and those that can not, in the form

$$
Q_{j}=-\frac{\partial V}{\partial q^{j}}+Q_{j}^{*}
$$

With this separation, the equations of motion take the form

$$
\begin{aligned}
I \ddot{\theta}-I \dot{\phi}^{2} \sin \theta \cos \theta+m g h \cos \theta \cos \phi-k h^{2}\left(\sin \theta \cos \theta \cos ^{2} \phi\right) & =Q_{\theta}^{*}, \\
I \sin ^{2} \theta \ddot{\phi}+2 I \sin \theta \cos \theta \dot{\theta} \dot{\phi}-m g h \sin \theta \sin \phi+k h^{2}\left(\sin \phi \cos \phi \sin ^{2} \theta\right) & =Q_{\phi}^{*} .
\end{aligned}
$$

In eq.7 $Q_{\theta}^{*}$ and $Q_{\phi}^{*}$ are generalized forces that can not be calculated simply by applying a transformation of coordinates to $F_{x}, F_{y}, F_{z}$ because they are components of a vector and depend on the reference system in which they are written. In order to calculate them, the virtual work done in virtual displacements $\delta \theta$ and $\delta \phi$ is calculated. First a virtual displacement $\delta \theta$ is performed keeping $\phi$ fixed.

$$
\delta w=\left(F_{z} \cos \theta-F_{x} \sin \theta\right) h \delta \theta
$$

The generalized forces are the coefficients that multiply the virtual displacements in order to get the virtual work so:

$$
Q_{\theta}^{*}=\left(F_{z} \cos \theta-F_{x} \sin \theta\right) h
$$

If a virtual displacement in the $\phi$ direction keeping $\theta$ fixed is performed, it is obtained

$$
Q_{\phi}^{*}=\left(-F_{z} \sin \phi-F_{y} \cos \phi\right) h
$$


And the equations of motion are then:

$$
\begin{gathered}
I \ddot{\theta}-I \dot{\phi}^{2} \sin \theta \cos \theta+m g h \cos \theta \cos \phi- \\
k h^{2}\left(\sin \theta \cos \theta \cos ^{2} \phi\right)-\left(F_{z} \cos \theta-F_{x} \sin \theta\right) h=0, \\
I \sin ^{2} \theta \ddot{\phi}+2 I \sin \theta \cos \theta \dot{\theta} \dot{\phi}-m g h \sin \theta \sin \phi+ \\
k h^{2}\left(\sin \phi \cos \phi \sin ^{2} \theta\right)+\left(F_{z} \sin \phi+F_{y} \cos \phi\right) h=0 .
\end{gathered}
$$

\section{Damping forces}

The damping forces in the fixed point are considered to vary linearly with the angular velocity in the form

$$
\begin{aligned}
& D_{\theta}=-\alpha_{\theta} h \dot{\theta} \\
& D_{\phi}=-\alpha_{\phi} h \dot{\phi}
\end{aligned}
$$

with $\alpha_{\theta}$ and $\alpha_{\phi}$ as dissipation constants. The equations of motion are finally written as

$$
\begin{gathered}
I \ddot{\theta}-I \dot{\phi}^{2} \sin \theta \cos \theta+m g h \cos \theta \cos \phi- \\
k h^{2}\left(\sin \theta \cos \theta \cos ^{2} \phi\right)-\left(F_{z} \cos \theta-F_{x} \sin \theta\right) h+\alpha_{\theta} h^{2} \dot{\theta}=0, \\
I \sin ^{2} \theta \ddot{\phi}+2 I \sin \theta \cos \theta \dot{\theta} \dot{\phi}-m g h \sin \theta \sin \phi+ \\
k h^{2}\left(\sin \phi \cos \phi \sin ^{2} \theta\right)+\left(F_{z} \sin \phi+F_{y} \cos \phi\right) h+\alpha_{\phi} h^{2} \dot{\phi}=0 .
\end{gathered}
$$

\section{Non-dimensional equations}

Selecting the diameter of the mast, $d$ as unit length and the magnitude of the inflow velocity in the $x$-direction, $u_{0}$ as characteristic velocity the non-dimensional variables are defined as $t^{*}=u_{0} t / d, x^{*}=x / d, y^{*}=y / d, z^{*}=z / d, \boldsymbol{u}^{*}=\boldsymbol{u} / u_{0}$. Using this set of variables the eqs. 1,2 and the system 14 can be written as follow:

$$
\begin{gathered}
\frac{\partial \boldsymbol{u}_{f}^{*}}{\partial t^{*}}+\left[\left(\boldsymbol{u}_{f}^{*}-\boldsymbol{u}_{m}^{*}\right) \cdot \nabla\right] \boldsymbol{u}_{f}^{*}-\frac{1}{R e} \nabla^{2} \boldsymbol{u}_{f}^{*}+\nabla p^{*}=\boldsymbol{f}^{*} \\
\nabla \cdot \boldsymbol{u}_{f}^{*}=0
\end{gathered}
$$




$$
\begin{aligned}
\ddot{\theta}-\dot{\phi}^{2} \sin \theta \cos \theta+\frac{m g d^{2} h}{u_{0}^{2} I} \cos \theta \cos \phi- \\
\frac{k d^{2} h^{2}}{u_{0}^{2} I}\left(\sin \theta \cos \theta \cos ^{2} \phi\right)-\frac{d^{2} h}{u_{0}^{2} I}\left(F_{z} \cos \theta-F_{x} \sin \theta\right)+\frac{d h^{2} \alpha_{\theta}}{u_{0} I} \dot{\theta}=0, \\
\sin ^{2} \theta \ddot{\phi}+2 \sin \theta \cos \theta \dot{\theta} \dot{\phi}-\frac{m g d^{2} h}{u_{0}^{2} I} \sin \theta \sin \phi+ \\
\frac{k d^{2} h^{2}}{u_{0}^{2} I}\left(\sin \phi \cos \phi \sin ^{2} \theta\right)+\frac{d^{2} h}{u_{0}^{2} I}\left(F_{z} \sin \phi+F_{y} \cos \phi\right)+\frac{d h^{2} \alpha_{\phi}}{u_{0} I} \dot{\phi}=0 .
\end{aligned}
$$

Here the time derivatives are taken with respect to the non-dimensional time. It is important to point out that the aerodynamic forces $F_{x}, F_{y}$ and $F_{z}$ result of the interaction of the mast with the fluid and are of the form

$$
F_{i}=\frac{1}{2} \rho_{f} u_{0}^{2} C_{i} A
$$

where the subindex $i$ represents $x, y$ or $z$ and $C_{i}$ is the force coefficient in the corresponding direction. The reference surface $A=d l$ is used with $l>h$ the length of the mast. Then, it is possible to write the external force term in 17 as

$$
\frac{2}{\pi m_{r}^{*}}\left(C_{z} \cos \theta-C_{x} \sin \theta\right)
$$

with the reduced mass coefficient defined as

$$
m_{r}^{*}=\frac{I /(d h)}{\pi(d / 2)^{2} l \rho_{f}}
$$

which compares the mass of fluid displaced by the cylinder with its moment of inertia, and acts as an effective mass of the system. Similarly, the non-dimensional parameter can be defined:

$$
m_{g r}^{*}=\frac{u_{0}^{2} I}{m g d^{2} h}
$$

that compares the influence of the gravity potential energy of the mast with the kinetic energy provided by the flow. Also, it is possible to define the reduced velocity

$$
u_{r}^{*}=\frac{2 \pi u_{0}}{\sqrt{k /\left(I / h^{2}\right)} d},
$$

which is the inverse of the natural frequency of the system scaled by the factor $u_{0} / d$. And for the damping forces the following non-dimensional parameter is defined

$$
\zeta_{i}^{*}=\frac{\alpha_{i}}{2 \sqrt{k I / h^{2}}}
$$


With all the previous in mind the system 17 is written in non-dimensional form as

$$
\begin{aligned}
& \ddot{\theta}-\dot{\phi}^{2} \sin \theta \cos \theta+\frac{\cos \theta \cos \phi}{m_{g r}^{*}}-\left(\frac{2 \pi}{u_{r}^{*}}\right)^{2}\left(\sin \theta \cos \theta \cos ^{2} \phi\right)- \frac{2}{\pi m_{r}^{*}}\left(C_{z} \cos \theta-C_{x} \sin \theta\right)+\frac{4 \pi \zeta_{\theta}^{*} \dot{\theta}}{u_{r}^{*}}=0, \\
& \sin ^{2} \theta \ddot{\phi}+2 \sin \theta \cos \theta \dot{\theta} \dot{\phi}-\frac{\sin \theta \sin \phi}{m_{g r}^{*}}+\left(\frac{2 \pi}{u_{r}^{*}}\right)^{2}\left(\sin \phi \cos \phi \sin ^{2} \theta\right)+ \\
& \frac{2}{\pi m_{r}^{*}}\left(C_{z} \sin \phi+C_{y} \cos \phi\right)+\frac{4 \pi \zeta_{\phi}^{*} \dot{\phi}}{u_{r}^{*}}=0 .
\end{aligned}
$$

This system of equations is very similar to the canonical case of the vortex induced vibrations (VIV) of a circular cylinder with two degrees of freedom ${ }^{5,10,22,33}$.

\section{E. FSI coupling}

In this contribution, a loosely coupled algorithm is employed. In one time step, the fluid mechanics problem is solved, the hydrodynamic forces on the cylinder are calculated and used to solve the rigid body equations; with the new location of the rigid body the fluid mesh is moved and the time step is advanced. Details on the coupling strategy and results of this scheme for a related VIV case can be found $\mathrm{in}^{10}$.

\section{NUMERICAL FORMULATION}

The following discretization schemes for the fluid and the rigid body mechanics problems are used: the Navier-Stokes equations are discretized using the stabilized finite element method, with Variational MultiScale stabilization. The unknowns of the problem are separated into grid scale components and subgrid scale components with the possibility of tracking the subgrid components in time and space to give more accuracy and stability to the numerical model. The momentum equation is separated from the continuity equation using the Schur complement for the pressure, each equation is solved independently and the solution of the coupled system is obtained in an iterative way. The time integration scheme used is a backward differentiation formula of second order with a variable time step of order $10^{-3}$, decreasing the time step size by a factor of four, produced no noticeable changes in the 
solution (not shown here for the sake of brevity). The details and validation of the numerical solution strategy are provided in ${ }^{34,35}$. The rigid body equations are integrated in time using a fourth order Runge-Kutta method. A similar set-up adequate for turbulent FSI cases was used in Pastrana et al. ${ }^{10}$ where validation and comparison with numerical and experimental results can be found.

\section{A. Boundary conditions and mesh properties}

As boundary conditions, at the inflow a uniform velocity is imposed; at the outflow a stress vector equals to zero is imposed. The bottom wall and the mast are considered no-slip surfaces while side and top walls of the wind tunnel are considered slip surfaces, see Fig. 1. The computational domain is discretized with 3.3 million unstructured elements clustered close to the cylinder. A distance of $0.09 d$ is covered with five layers of elements starting from the cylinder's surface in order to solve the laminar boundary layer. The simulations were run using two supercomputing facilities: Marenostrum IV (using 144 cores) at Barcelona Supercomputing Center and Miztli (using 128 cores) at 'Universidad Nacional Autónoma de México'. A typical simulation was completed within 43 hours.

In order to assess on the grid sensitivity of the numerical solution, two different computational meshes were used to solve a representative case. The first mesh was the $3.3 \mathrm{M}$ one described above, the second one was a refined $25 \mathrm{M}$ elements mesh obtained using the mesh multiplication technique presented by Houzeaux et. al. ${ }^{36}$. The difference on the maximum average amplitude of the oscillation in the cross-flow direction is less than $3 \%$ (this result is shown in Fig. 4 of Sec.IV A). Differences in other relevant quantities are of the same order of magnitude. Thus, the 3.3M elements mesh is considered adequate to accurately solve the proposed problem.

\section{NUMERICAL RESULTS}

The flow at two Reynolds numbers of $R e=100,200$ and several combinations of reduced velocity and reduced mass is analyzed. First, at $R e=100$, the influence of the reduced velocity on the response of the system for a fixed value of $m_{r}^{*}=5$ is explored, i.e. $u_{r}^{*}=$ $3,3.4,3.5,4,5,6,7,8,9,10,11$. Moreover, the influence of the reduced mass on the 
dynamical behavior of the mast for a fixed value of $u_{r}^{*}=5$ is studied at mass ratios of $m_{r}^{*}=3,5$ and 7 . Also, to give a first glance in the effect of the Reynolds number on the system response two cases at $R e=200, m_{r}^{*}=5$ and $u_{r}^{*}=5,7$ are also considered. Finally, the case with the fixed cylinder is also included for comparison purposes. This accounts for a total of 16 cases where the response of the system is analyzed. In all movable cases the mass damping ratio $4 \pi \zeta_{\phi}^{*} / u_{r}^{*}$ has been fixed to 0.01 . Without loss of generality, the influence of gravity is neglected and attention is focused on the balance between the aerodynamic forces and the restoring forces of the spring.

As initial condition for the flow, a developed velocity field with $R e=100$ and the mast fixed was chosen for cases with $u_{r}^{*}=3, m_{r}^{*}=5$, and $u_{r}^{*}=5, m_{r}^{*}=7$. Then, the FSI simulation was started and evolved until a final sustained oscillatory state or a final steady state was reached. The oscillatory state of the case with $u_{r}^{*}=5, m_{r}^{*}=7$ was used as initial condition for the rest of cases.

\section{A. Cylinder displacement}

The displacement of the tip of the mast at $R e=100$, in the $x^{*}$ and $y^{*}$ directions is shown in Figure 3. Final steady or oscillatory states are observed depending on the physical parameters. Small values of the reduced mass $m_{r}^{*}=3$ and large values of the reduced velocity $u_{r}^{*}=7,9,11$ produce large deviations from the vertical axis, that can be quantified with the displacement of the tip of the mast in the $x^{*}$-direction. This is reasonable given that, on the one hand, small values of the reduced mass are obtained when the mass of fluid displaced by the cylinder is large compared with its moment of inertia (which can be interpreted as the fluid being much heavier than the solid). On the other hand, large values of the reduced velocity are related to small values of the natural frequency of the system, which can be associated to relatively small values of the restoring forces of the spring.

The effect of the reduced velocity on the final steady or oscillatory trajectory of the system for constant $m_{r}^{*}=5$ can be observed in Fig. 3. No significant amplitude is present for $u_{r}^{*}=3$. The onset of the oscillations typical of the $I$ branch is observed for $u_{r}^{*}=3.4,3.5$. The trajectories of the upper branch are observed for $u_{r}^{*}=4,5,6,7$ with the maximum amplitudes for this value of $m_{r}^{*}$ at $u_{r}^{*}=5,6$. A gradual decrement in the trajectory amplitude is observed for larger $u_{r}^{*}$ with the minimum displacement obtained in the lower branch for 


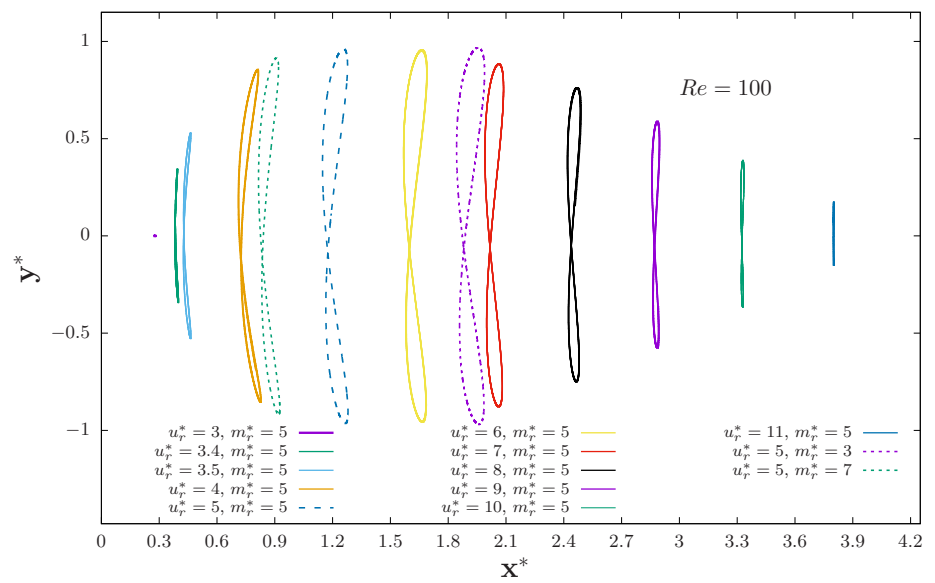

FIG. 3: Final trajectory dependence on the reduced velocity $u_{r}^{*}$ and the reduced mass $m_{r}^{*}$ for cases with $R e=100$.

$u_{r}^{*}=11$.

Similarly, the effect of the reduced mass on the oscillatory final state of the system is also shown in Fig. 3 for constant $u_{r}^{*}=5$ and $m_{r}^{*}=3,5,7$. It is noticed that as the reduced mass increases, the deviation of the cylinder from the vertical axis decreases and smaller oscillations in the $x^{*}$ and $y^{*}$-directions are obtained. Moreover, less significant variations in the $y^{*}$-direction amplitude compared to those obtained varying the reduced velocity are observed. This is reasonable given that the reduced velocity is directly related to the nondimensional frequency of the system.

The amplitude and frequency of the oscillations vary with the physical parameters. Their average values around the mean displacement $\bar{x}^{*}$ and $\bar{y}^{*}$ for the last four cycles (in the $y^{*}$-direction) of the regular oscillatory state are reported in Table I. Also, a graphical representation of the table can be seen in Fig. 4 for the $y^{*}$-direction amplitudes together with the experimental results for 2dof elastically mounted cylinders of Jauvtis and Williamson ${ }^{5}$ $(\mathrm{J} \& \mathrm{~W})$, and the pivoted configuration of Flemming and Williamson ${ }^{7}(\mathrm{~F} \& \mathrm{~W})$. Also, the numerical results of Bourguet and Lo Jacono ${ }^{17}$ (B \& LJ) and Zhao et. al. ${ }^{18}$ for 2 dof elastically mounted cylinders in the laminar regime, with $R e=100, m_{r}^{*}=10$ and $R e=150, m_{r}^{*}=2$, respectively, are included for comparison. In the experimental elastically mounted case, 


\begin{tabular}{c|c|c|c}
$u_{r}^{*}\left[m_{r}^{*}\right]$ & $\bar{x}^{*}$ [freq.] & $\bar{y}^{*}$ [freq.] & $\bar{z}^{*}$ [freq.] \\
\hline $3[5]$ & $0.280 \pm 0.000[-]$ & $0.0 \pm 7 \times 10^{-4}[0.102]$ & $10.496 \pm 0.000[-]$ \\
$3.4[5]$ & $0.390 \pm 0.007[0.320]$ & $0.000 \pm 0.343[0.161]$ & $10.490 \pm 0.003[0.323]$ \\
$3.5[5]$ & $0.442 \pm 0.016[0.315]$ & $0.000 \pm 0.488[0.157]$ & $10.485 \pm 0.006[0.315]$ \\
$4[5]$ & $0.763 \pm 0.051[0.309]$ & $0.000 \pm 0.849[0.155]$ & $10.455 \pm 0.021[0.310]$ \\
$5[5]$ & $1.210 \pm 0.062[0.278]$ & $-0.006 \pm 0.950[0.141]$ & $10.406 \pm 0.028[0.278]$ \\
$6[5]$ & $1.631 \pm 0.063[0.250]$ & $0.000 \pm 0.954[0.125]$ & $10.350 \pm 0.028[0.250]$ \\
$7[5]$ & $2.038 \pm 0.048[0.226]$ & $0.002 \pm 0.881[0.113]$ & $10.281 \pm 0.025[0.226]$ \\
$8[5]$ & $2.453 \pm 0.030[0.210]$ & $0.006 \pm 0.756[0.104]$ & $10.195 \pm 0.019[0.210]$ \\
$9[5]$ & $2.877 \pm 0.019[0.196]$ & $0.006 \pm 0.581[0.097]$ & $10.089 \pm 0.012[0.194]$ \\
$10[5]$ & $3.326 \pm 0.007[0.184]$ & $0.011 \pm 0.377[0.091]$ & $9.955 \pm 0.005[0.184]$ \\
$11[5]$ & $3.803 \pm 0.001[0.172]$ & $0.010 \pm 0.166[0.086]$ & $9.786 \pm 0.001[0.172]$ \\
$5[3]$ & $1.941 \pm 0.075[0.257]$ & $-0.003 \pm 0.989[0.128]$ & $10.294 \pm 0.035[0.257]$ \\
$5[7]$ & $0.870 \pm 0.055[0.292]$ & $-0.001 \pm 0.915[0.147]$ & $10.443 \pm 0.024[0.294]$
\end{tabular}

TABLE I: Mean value of non-dimensional amplitude $\left(\bar{x}^{*}, \bar{y}^{*}\right.$, and $\left.\bar{z}^{*}\right) \pm$ maximum displacement in $x^{*}, y^{*}$ and $z^{*}$ directions calculated for the last four oscillation cycles in the quasi-periodic state for cases with $R e=100$.

the authors used $R e=1000-15000$. The experimental pivoted configuration considered cylinders with $A R=31,41$, at $R e 1000$ that were allowed to oscillate in two angular degrees of freedom. It is important to mention that the experimental results were reported using a different definition of the reduced velocity from the one used in the present study. Thus, experimental data was scaled according to the empirical relation $f_{w} / f_{n}=\sqrt{m_{r}^{*} /\left(m_{r}^{*}+C_{a}\right)}$, where $f_{n}$ is the natural frequency of the system in vacuum, $f_{w}$ is the natural frequency of the system in water and $C_{a} \simeq 1$ is the added mass coefficient for cylinders with uniform mass distribution.

The overall dependence of the maximum amplitude on the reduced velocity of the considered system resembles remarkably the experimental results of $\mathrm{F} \& \mathrm{~W}$ and the results of the 


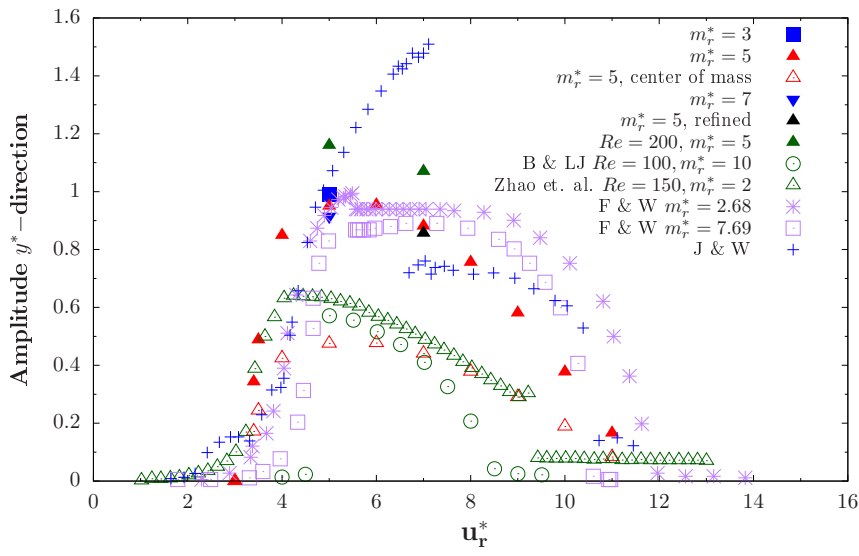

FIG. 4: Average amplitude in $y^{*}$-direction for the last four oscillation cycles in the cross-flow direction for all the considered cases. Experimental results for a 2 dof system from $J \& \mathrm{~W}^{5}$, and for a pivoted configuration $\mathrm{F} \& \mathrm{~W}^{7}$ together wit the numerical results of laminar cases of B\&LJ ${ }^{17}$ and Zhao et. al. ${ }^{18}$ are also included as references.

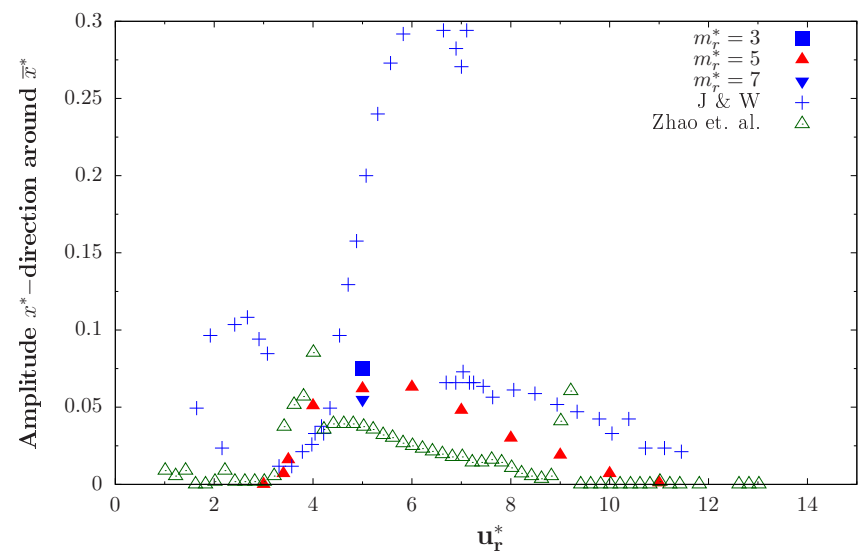

FIG. 5: Average maximum amplitude in the in-line direction for the last four oscillation cycles for $R e=100$ around $\bar{x}^{*}$. Experimental results for a 2 dof system from $\mathrm{J}_{\mathrm{W}} \mathrm{W}^{5}$ together wit the numerical results of Zhao et. al. ${ }^{18}$ are also plotted. 
laminar cases. The relatively larger maximum amplitudes obtained in our results are due to the aspect ratio of the cylinder $A R=10$ and the presence of the fixed point. The displacement of the center of mass of the pivoted cylinder compares reasonably well with the results obtained in the reference cases and is also included in Fig. 4. A three branches response is observed: the initial branch $(I)$, which is the initial excitation region; the upper branch $(U)$, where the maximum amplitudes appear; and the lower branch $(L)$, where moderate amplitudes are observed. Comparing the laminar cases, it is noticed from Figure 4 that the pivoted cylinder presents a 'wider' upper branch than those elastically mounted. This suggests that the pivoted configuration is able to oscillate in a larger range of frequencies than the elastically mounted one. A possible explanation is that, given that there is a fixed point, increasing amplitudes are observed from the base to the tip of the mast, allowing the system to have multiple frequencies through the cylinder's axis to be excited.

Fair qualitatively agreement in the cylinder dynamic response is also obtained comparing the pivoted configurations of the present study with the experimental work of F\&W. The deviations, apart from the Reynolds numbers dependence, can be explained considering the differences between the experimental and numerical set-up. In the experiment the tip of the cylinder oscillated near the bottom of a water tank with a clearance of $2 \mathrm{~mm}$, and the fixed point was above the free surface. Thus, interaction between the tip vortex and the boundary layer at the bottom of the tank is present in the experimental system, while in the present study the boundary layer at the bottom interacts with the lower section of the cylinder, where the fixed point is located. Also, in the experimental set-up gravity tends to oppose the deviations of the cylinder from the vertical axis. In the present numerical work, the gravity effect is not considered. At this point, it is important to insist in the fact that the relatively large amplitudes obtained in this low $R e$ system are due to the aspect ratio of the cylinder $A R=10$, and that they do not mean that this system can produce larger oscillation amplitudes than its high Re counterparts. To illustrate this point, the maximum absolute value for the angle in the cross flow direction is $|\phi|=0.090$.

The region of extra amplification of the oscillations present in the 2 dof configuration of J\&W observed in Fig. 4, known as the super upper branch $(S U)$ is not found in the present pivoted configuration for the analyzed parameters. In order to explore if the $S U$ branch can be found with an increased value of the Reynolds number, the cases with $R e=200$ and $u_{r}^{*}=5,7$ with $m_{r}^{*}=5$ are considered and plotted also in the figure. An extra amplification 
with respect to their $R e=100$ counterparts is obtained but no evidence of the $S U$ branch is found. In the authors' opinion this difference can be explained taking into account the $R e$ dependence and the fact that in the studied cases the system never reaches a resonant state, as will be shown in Sec. V.

The oscillation amplitude in the $x^{*}$-direction for cases with $R e=100$ and $m_{r}^{*}=5$ is shown in Fig. 5, where the experimental result for the 2dof configuration of J\&W and the numerical results of Zhao et. al. ${ }^{18}$ are also included for comparison. It can be observed that the laminar cases follow the same trends, and that similar amplitudes are obtained for values of $u_{r}^{*} \leq 4$ for all cases. Relatively small amplitudes are obtained in the pivoted configuration for the range $4<u_{r}^{*}<7$ with respect to the experimental result, where the $\mathrm{SU}$ branch is present. Despite the noticeable deviations from the vertical axis of the mast in this direction, a narrow trajectory is obtained in the pivoted case, and consequently the system response is closer to that of the 1 dof configuration, where no $S U$ branch has been reported.

\section{B. Hydrodynamic force coefficients}

The hydrodynamic force coefficients on the cylinder were calculated. The mean and rootmean-square (RMS) values for the coefficients in the $x^{*}$, and $z^{*}$ directions together with the

RMS value in the $y^{*}$-direction and the mean value of the $\theta$ angle can be found in Table II for all the considered cases with $R e=100$. Time averages were taken in the last four cycles of the quasi-periodic state, and are denoted with an overline. General trends and a detailed description for selected cases follows.

The fixed and the quasi-static case $\left(u_{r}^{*}=3, m_{r}^{*}=5\right)$ have very similar values in the force coefficients, as expected given the extremely small amplitude of the oscillations and inclination angle, and can be compared with similar configurations of fixed, finite length vertical cylinder. In particular the flow around a cantilevered cylinder with the same $A R$ and $R e$ was investigated by Liu et. al. ${ }^{37}$. The authors presented a span distribution of the mean value of the drag coefficient and reported a fairly constant region around $\bar{C}_{D}=1.25$, which is noticeably larger than the present value of $\bar{C}_{D}=0.932$ obtained for the fixed case. This is considered a reasonable difference because in the pivoted configuration employed in this work, there is a clearance in the bottom of the cylinder (see Fig. 1) which induces a different 


\begin{tabular}{c|c|c|c|c|c|c}
$u_{r}^{*}\left[m_{r}^{*}\right]$ & $\bar{C}_{x}$ & $C_{x r m s}$ & $C_{y r m s}$ & $\bar{C}_{z}$ & $C_{z r m s}$ & $\bar{\theta}$ \\
\hline fixed & 0.932 & $2 \times 10^{-4}$ & $4 \times 10^{-3}$ & -0.020 & $5 \times 10^{-5}$ & 1.570 \\
$3[5]$ & 0.920 & $2 \times 10^{-4}$ & $4 \times 10^{-4}$ & -0.002 & $4 \times 10^{-4}$ & 1.544 \\
$3.4[5]$ & 0.997 & 0.005 & 0.430 & -0.002 & 0.010 & 1.534 \\
$3.5[5]$ & 1.060 & 0.008 & 0.567 & -0.005 & 0.018 & 1.530 \\
$4[5]$ & 1.417 & 0.045 & 0.680 & -0.032 & 0.045 & 1.498 \\
$5[5]$ & 1.430 & 0.060 & 0.400 & -0.050 & 0.032 & 1.455 \\
$6[5]$ & 1.324 & 0.050 & 0.230 & -0.073 & 0.020 & 1.414 \\
$7[5]$ & 1.210 & 0.036 & 0.128 & -0.094 & 0.012 & 1.375 \\
$8[5]$ & 1.103 & 0.023 & 0.066 & -0.115 & 0.006 & 1.335 \\
$9[5]$ & 1.010 & 0.012 & 0.028 & -0.136 & 0.003 & 1.293 \\
$10[5]$ & 0.929 & 0.004 & 0.007 & -0.160 & 0.001 & 1.248 \\
$11[5]$ & 0.855 & $8 \times 10^{-4}$ & $6 \times 10^{-4}$ & -0.185 & $2 \times 10^{-4}$ & 1.200 \\
$5[3]$ & 1.355 & 0.034 & 0.278 & -0.107 & 0.025 & 1.384 \\
$5[7]$ & 1.440 & 0.077 & 0.488 & -0.022 & 0.035 & 1.487
\end{tabular}

TABLE II: Mean value of force coefficients $\bar{C}_{x}$, and $\bar{C}_{z}$, and their root mean square values, $C_{x r m s}, C_{y r m s}$ and $C_{z r m s}$, together with $\theta$ angle, for the last four oscillation cycles in the quasi-periodic state for cases with $R e=100$.

flow configuration than the one in the cantilevered case, where there is no clearance at all. Taking this geometrical characteristic into account, the result obtained is also compared with the mean drag coefficient for the flow around a circular cylinder with the same Re, but larger $A R=50$, and with two free-ends ${ }^{8}$. The authors reported $\bar{C}_{D}=1.001$ which compares reasonably well with the obtained values in the present study. 


\section{Effect of the reduced velocity with constant $m_{r}^{*}=5$}

For the cases with $m_{r}^{*}=5$, it is observed that $\bar{C}_{x}$ increases as the amplitude of the oscillations increases with the reduced velocity up to $u_{r}^{*}=5$, where it reaches its maximum value of $\bar{C}_{x}=1.430$; this represents an increment of $36 \%$ with respect to the quasi-static case $u_{r}^{*}=3$. In the maximum amplitude case $u_{r}^{*}=6, \bar{C}_{x}$ decreases approximately $8 \%$ with respect to its maximum value. It is noticed that the difference in the oscillation amplitudes between the cases with $u_{r}^{*}=5$ and $u_{r}^{*}=6$ is very small (less than $3 \%$ ) and that the difference in $\bar{C}_{x}$ is mainly due to the smaller inclination angle $\bar{\theta}$ (larger deviation from the vertical) in the latter case. As $u_{r}^{*}$ increases further, the deviation from the vertical of the mast increases as well, and the $\bar{C}_{x}$ coefficient decreases accordingly. The RMS values of $C_{x}$ are small compared with unity in general. However the $I$ and $L$ branches show values at least one order of magnitude smaller than those obtained in the $U$ branch, where the maximum $C_{x r m s}=0.060$ is reached for $u_{r}^{*}=5$. In general the variation of $C_{x r m s}$ follows the same trends as the dependence of the maximum displacements in the $x$-direction with $u_{r}^{*}$ exposed in Sec. IV A.

In the $y^{*}$-direction, large values of $C_{y r m s}$ are obtained for cases in the $I$ and the beginning of the $U$ branches. The maximum value, $C_{y r m s}=0.680$, is obtained for $u_{r}^{*}=4$ and a rapid decay in $C_{y r m s}$ is observed as $u_{r}^{*}$ increases. It is noticeable that the maximum displacement in the $y^{*}$-direction occurs for $u_{r}^{*}=6$ with a rather small value of $C_{y r m s}=0.230$. This can be understood recalling the general solution of the classical one-dimensional forced-damped oscillator $^{38}$, where the oscillation amplitude is proportional to the quotient of the forcing magnitude to the difference between the forcing frequency and the natural frequency of the system. In the present pivoted configuration, the named quotient is maximum when $u_{r}^{*}=5,6$. Moreover, the inclination angle of the cylinder also affects the forcing of the system through the $C_{z}$ force coefficient. In order to obtain a deeper understanding of the relation between the forces applied and the VIV of the pivoted cylinder, a detailed analysis of the linearized form of eqs. 24 is suggested, the first steps in this direction are presented in Sec. V and will be extended in future studies.

The absolute value of $\bar{C}_{z}$ follows similar trends as $\bar{C}_{x}$ for $u_{r}^{*}=3.4,3.5,4,5$ in the $I$ and $U$ branches, but with smaller values. However as $u_{r}^{*}$ increases further, $\bar{\theta}$ diminishes and a considerable increment in the absolute value of $\bar{C}_{z}$ is obtained, reaching its maximum 
$\left|\bar{C}_{z}\right|=0.185$ for $u_{r}^{*}=11$. Thus, a noticeable influence in the cross-flow oscillation amplitude is observed, see eqs. 24. It should be pointed out that the sign of $\bar{C}_{z}$ is negative, as expected from the inclination of the body with respect to the mean flow.

\section{Effect of the reduced mass with constant $u_{r}^{*}=5$}

The $\bar{C}_{x}$ value has small variations with respect to the reduced mass parameters considered in the present study, with a maximum difference of $6 \%$. The maximum value of $\bar{C}_{x}=1.440$ is reached for $u_{r}^{*}=5, m_{r}^{*}=7$. As before, $\bar{C}_{x}$ decreases as the inclination angle of the mast $\bar{\theta}$ decreases (with smaller values of $m_{r}^{*}$ ). Following a similar dependency with the inclination angle $C_{x r m s}$ decreases with decreasing values of the reduced mass, with a maximum value of 0.077 for $m_{r}^{*}=7$ and a minimum value of 0.034 for $m_{r}^{*}=3$.

It is observed that $C_{y r m s}$ decreases with decreasing values of the reduced mass with a maximum value of 0.488 for $m_{r}^{*}=7$ and a minimum value of 0.278 for $m_{r}^{*}=3$. It is pointed out that even when there is a significant difference in the RMS value of the force coefficient in the $y^{*}$-direction the differences in the maximum amplitude obtained are relatively small. This can be explained recalling again the solution of the one-dimensional forced-damped oscillator $^{38}$, where the oscillation amplitude is proportional to the quotient of the forcing magnitude to the reduced mass of the system. Thus, as long as this quotient does not vary significantly, small variations in the resulting amplitudes are to be expected.

The absolute value of $\bar{C}_{z}$ increases with decreasing values of the reduced mass, again related to larger deviations from the vertical axis of the cylinder, the maximum is $\left|\bar{C}_{z}\right|=$ 0.107 for $m_{r}^{*}=3$ and the minimum value is $\left|\bar{C}_{z}\right|=0.022$ for $m_{r}^{*}=7$.

\section{Dominant frequencies and phase-shift}

A smooth and regular behavior is obtained for the fluctuations around the mean values of the hydrodynamic force coefficients on the cylinder. Their energy spectral density (ESD) reported as a function of the quotient $S t / S t_{f}$, where $S t_{f}=0.102$ is the Strouhal number of the quasi-static case, is shown in Fig. 6 for selected cases. From the ESD it can be observed that as the reduced velocity is increased, the Strouhal number in the $y^{*}$-direction decreases and approaches the Strouhal number of the quasi-static case (Figs. 6a, 6c, 6e, 6f). A less 
significant influence in the oscillation frequency is observed as the reduced mass is increased (see Figs. 6b, 6c, 6d), in accordance with the less significant effect of $m_{r}^{*}$ on the maximum oscillation amplitudes discussed in Sec. IV A and the previous section.

Additionally, the phase-shift between the displacements and the force coefficients was approximated using the maximum value of the correlation function between displacements and force coefficient in the respective directions. For all cases, there is a phase-shift very close to $\pi$ in the $x^{*}$-direction. In the $y^{*}$-direction very small phase-shifts are present for all cases, except for the one in the $L$ branch, which has a phase difference of $0.3 \pi$. This behavior is due to the small value of the damping parameter used, that makes phase-differences only possible when the non-synchronization region is reached. This will be further discussed in Sec. V. In the $z^{*}$-direction small phase-shifts are obtained in general, and only noticeable shifts in the range $0.1 \pi-0.25 \pi$ are obtained for cases with $u_{r}^{*}>7$ with $m_{r}^{*}=5$.

\section{Vortex shedding pattern}

The vortex shedding process is illustrated, without loss of generality, for the case $u_{r}^{*}=5$, $m_{r}^{*}=7$ in Fig. 7, and a typical flow structure is shown in Fig. 8. The non-dimensional pressure distribution on the cylinder surface and vortical structures represented by contours of $y^{*}$-vorticity are depicted for half of the cycle (in the figure the time is expressed in fraction of a vortex shedding cycle $\tau$ ), a positive value of the $y^{*}$-vorticity of 0.3 is shown in yellow and a negative one of -0.3 is shown in dark purple. Figure 7 a at $0.125 \tau$ shows the beginning of the process where the shear layer roll-up is about to occur and the tip of the cylinder is at is maximum $y^{*}$-elongation. As the process continues, the tip of the cylinder moves towards $y^{*}$-negative values and the vortex separates from the shear layer being shed into the wake. As in the fixed cylinder ${ }^{37,39}$, there is an uneven distribution of the pressure along the cylinder axis, the low-pressure in the rear end of the cylinder decreases towards the cylinder free-end (see pressure profile in Fig. 7) which favors the acceleration of the flow at the tip of the cylinder and the formation of the tip vortex. This acceleration of the flow initiates a downwash process, similar to the one described by Krajnovic ${ }^{39}$ for the fixed cylinder, with flow structures bending towards the ground. At the same time, there is a fluid entrainment at the bottom of the cylinder which goes up as a result of the interaction with the ground and the suction effect produced by the low-pressure zone at the top half of the cylinder. 


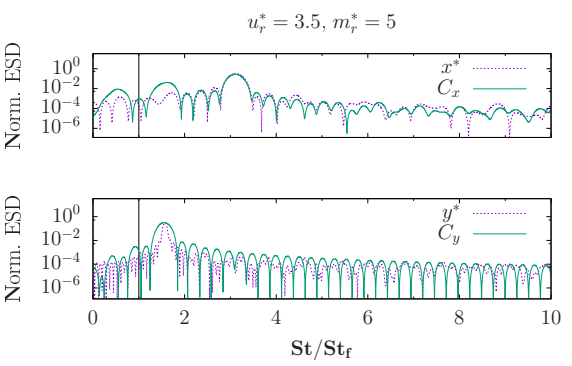

(a)

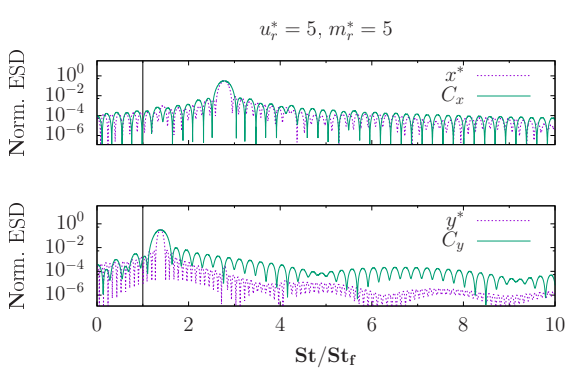

(c)

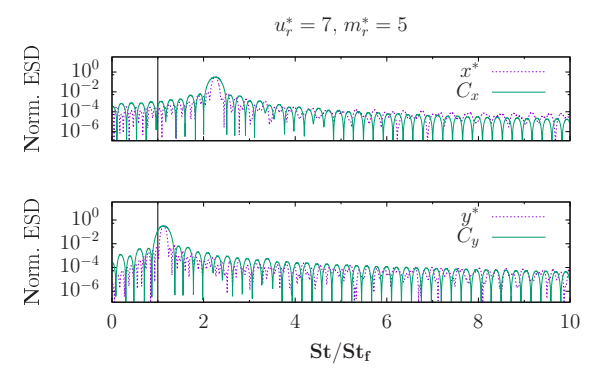

(e)

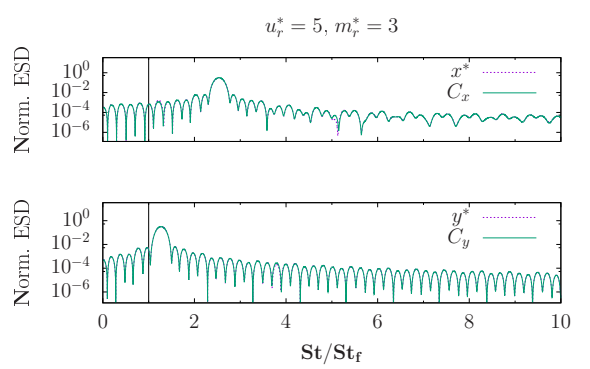

(b)

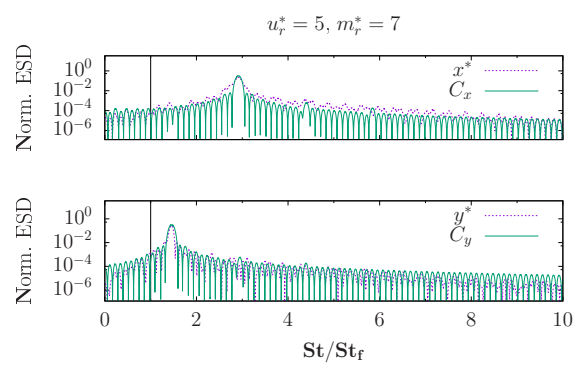

(d)

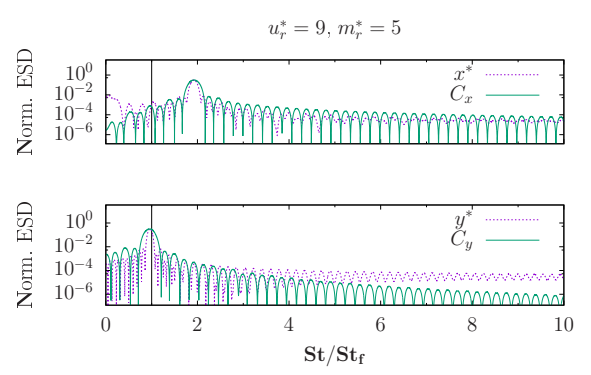

(f)

FIG. 6: Normalized energy spectrum density of $x^{*}$ and $y^{*}$ components of the displacement of the tip of the mast, and hydrodynamic forces coefficients $C_{x}$ and $C_{y}$ for (a) $u_{r}^{*}=3.5$, $m_{r}^{*}=5$, (b) $u_{r}^{*}=5, m_{r}^{*}=3$, (c) $u_{r}^{*}=5, m_{r}^{*}=5$, (d) $u_{r}^{*}=5, m_{r}^{*}=7$, (e) $u_{r}^{*}=7, m_{r}^{*}=5$ and (f) $u_{r}^{*}=9, m_{r}^{*}=5 . S t / S t_{f}=1$ is highlighted with a vertical solid line. 
When the separated shear layer rolls-up, it starts at the cylinder free-end following the downwash process (see Fig. 8, where this process and the flow structures are annotated), and due to the interaction between the two ends of the cylinder, the vortex shedding occurs in an asynchronous way from top to bottom along the cylinder axis. Thus, the vortex cores formed, instead of being shed parallel to the cylinder axis as in the infinite cylinder (see for instance $\left.{ }^{40,41}\right)$, they follow an oblique angle, which increases due to the acceleration at the tip as vortices travel down in the wake (see Figs. 8 and 9).

Depending on the reduced velocity, different morphological properties in the coherent structures can be appreciated. Instantaneous coherent structures for selected cases are plotted in Figs. 9, where iso-surfaces of the Q-criterion colored with the velocity magnitude are presented. First, as the vortex shedding is done out of phase along the cylinder axis, vortex dislocations in the upper and lower sections are observed (see Fig. 9). Second, as the inclination angle increases, more elongated structures are obtained, see the sequence of Figs. 9c-9b-9a, where the mean value of the $\theta$ angle goes from $\bar{\theta}=1.487$ to $\bar{\theta}=1.384$ (smaller values of $\bar{\theta}$ correspond to larger inclination angles, see Fig. 2). It is pointed out that despite the different elongations, the coherent structures obtained share general characteristics and are very similar to hairpin vortices. Nonetheless, the case with $u_{r}^{*}=5, m_{r}^{*}=7$ has an important morphological difference in the wake (see Fig. 9c). A reversed flow region behind the upper section of the cylinder is suggested by the deformation of the structures of the wake. The vortices remain closer to the cylinder than their counterpart in the other cases and a clear bending towards the cylinder is observed in the upper section of the second, third and fourth coherent structures shown in Fig. 9c.

A typical vortex shedding pattern can be observed in Fig. 10 for $u_{r}^{*}=5, m_{r}^{*}=3$. Snapshots of half a cycle of the cylinder displacement are shown with stream traces colored with the $z^{*}$-component of the vorticity. Iso-surfaces of the Q-criterion are shown with shaded areas to help the visualization of the three-dimensional structures. The upper section of the sub-figures show a vertical view of the stream traces, while the lower section show a side view of the same. A clockwise recirculation region grows in extension Figs. 10a-10b and is finally shed Figs. 10b-10c, while the counter-clockwise rotating vortex attached to the cylinder grows in extension and strength. The beginning of the next half cycle is shown in Fig. 10f. It is pointed out that the vortex detaches when the cylinder travels its way back to $y^{*}=0$ (Fig. 10c), after the maximum amplitude has been reached. Moreover, the phase 

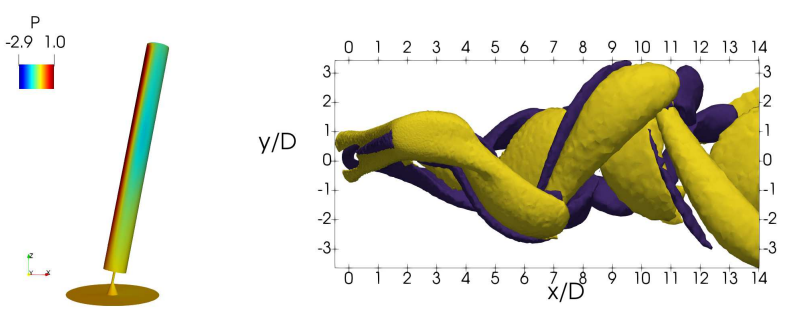

(a)
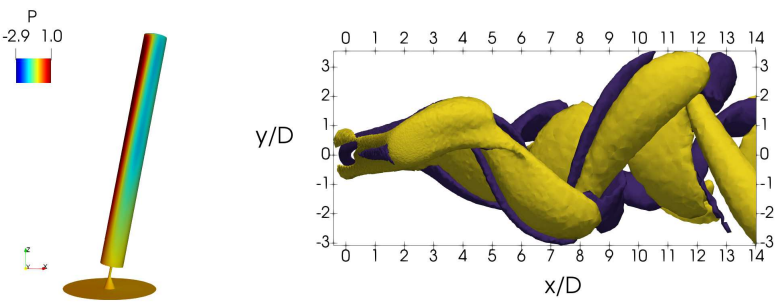

(b)
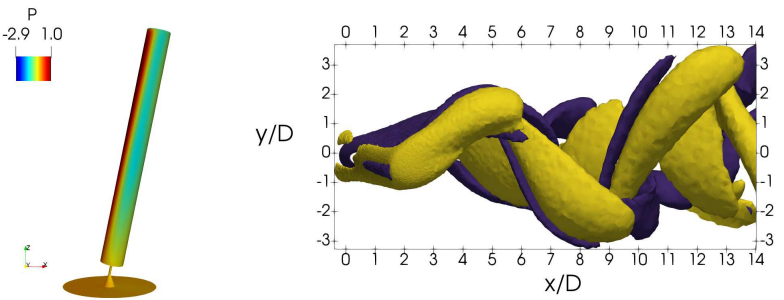

(c)
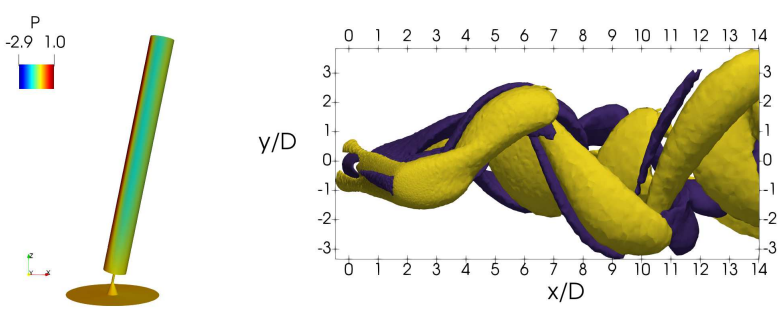

(d)

FIG. 7: (left) Non-dimensional pressure distribution (right) Non-dimensional instantaneous contours of y-vorticity ( +0.3 in yellow and -0.3 in dark purple) during half of a cycle for $u_{r}^{*}=7$ and $m_{r}^{*}=5$.

(a) $0.125 \tau$, (b) $0.25 \tau$, , (c) $0.375 \tau$, (d) $0.5 \tau$ 


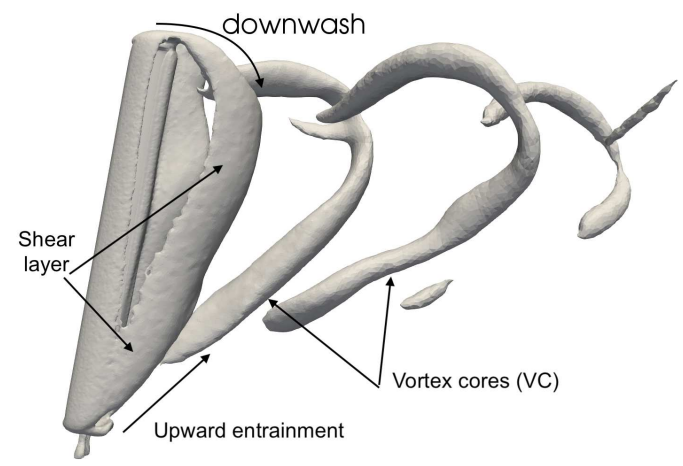

FIG. 8: Typical flow structure obtained. The downwash region, the shear layer instantaneous location, the upward entrainment section and the vortex cores are highlighted.

difference in the shedding along the cylinder axis can be clearly seen in the lower part of Figs. 10b-10d, where the clockwise recirculation region is observed to detach from the upper section of the mast. The second half of the cycle, where the mast moves in the increasing direction of $y^{*}$ shows similar trends (not shown here).

The regions where vortical structures shed from opposite sides of the cylinder merge are shown as co-rotating vortex pairs being convected down together; the Q-criterion iso-surfaces clearly show this for the counter-clockwise rotating vortex in the upper part of Fig. 10a. As the flow evolves, the vortices travel downstream and are eventually dissipated. This vortex pattern has been observed experimentally and was reported as the $2 \mathrm{C}$ mode ${ }^{7}$; to the authors' knowledge, this is the first time such a detailed visualization of the structure and origin of this pattern is provided in the VIV context for pivoted cylinders. It should be pointed out that as the strong vortex attached to the cylinder elongates before being shed from the upper section of the cylinder, a small portion of the vortical structure separates and is convected downstream independently of the main large structure (see Figs. 10d-10f).

Details on the vortex dynamics near the cylinder surface for half oscillation cycle can 

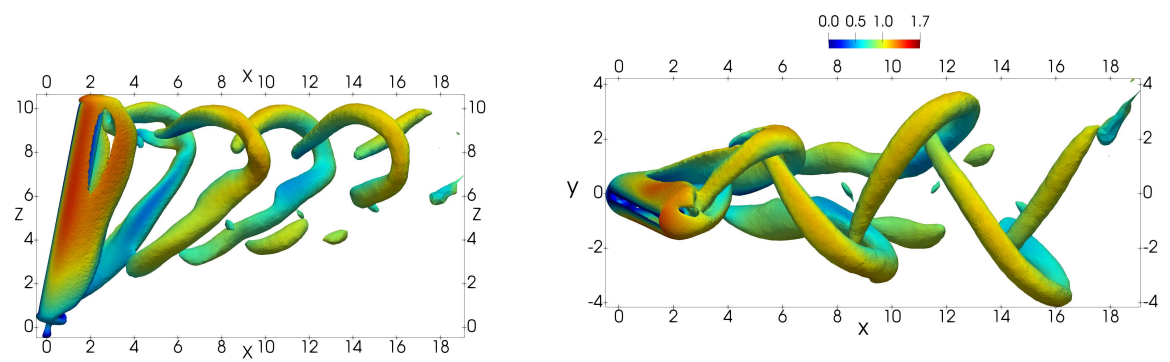

(a)
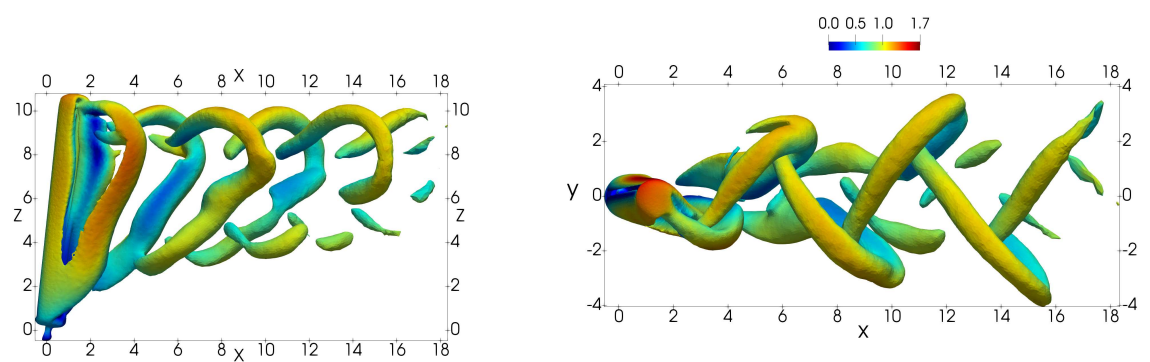

(b)
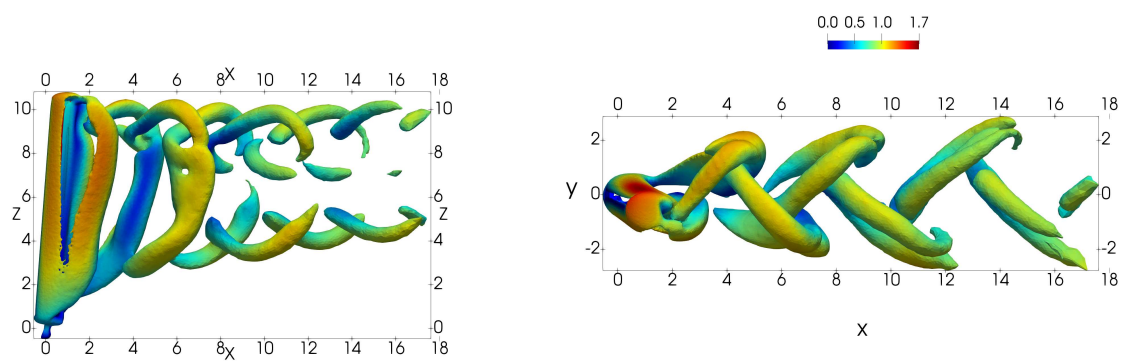

(c)

FIG. 9: Lateral (left) and top (right) views of the vortex shedding pattern shown by instantaneous $Q$ iso-surfaces $Q=0.1 u_{0}^{2} / d^{2}$ colored by the velocity magnitude for a) $u_{r}^{*}=5, m_{r}^{*}=3$, b) $u_{r}^{*}=5, m_{r}^{*}=5$, c) $u_{r}^{*}=5, m_{r}^{*}=7$. 


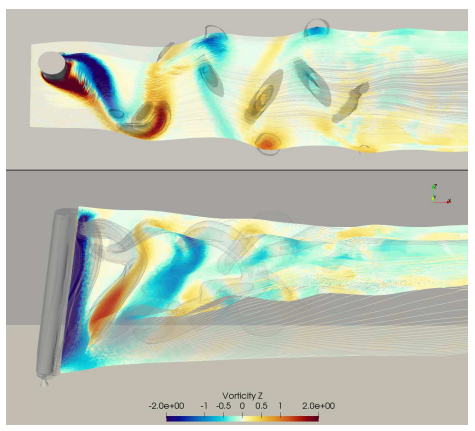

(a)

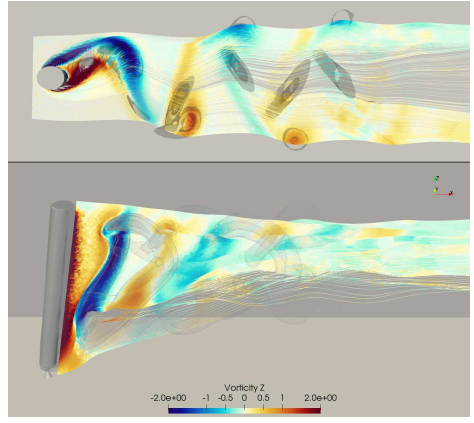

(c)

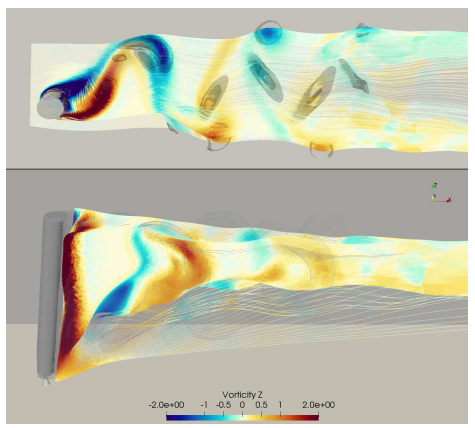

(e)

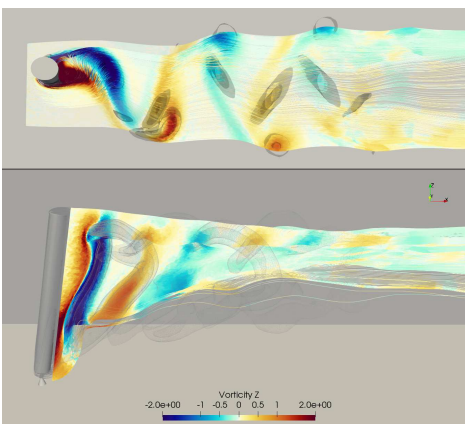

(b)

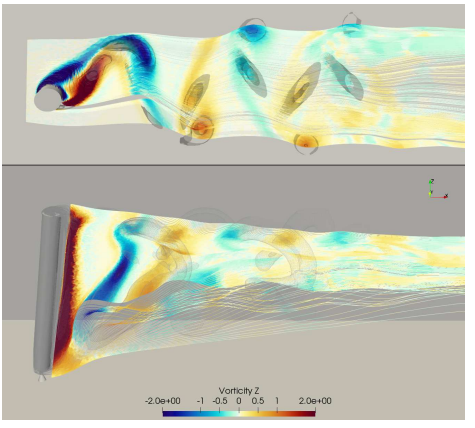

(d)

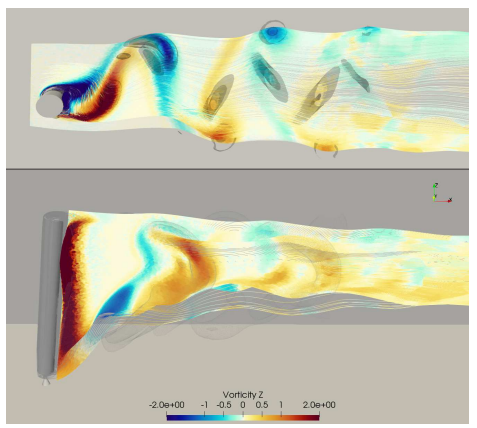

(f)

FIG. 10: Stream traces colored with $z^{*}$ vorticity values for $u_{r}^{*}=5, m_{r}^{*}=3$ at a) $0.02 \tau$, b) $0.14 \tau$, c) $0.25 \tau$, d) $0.37 \tau$, e) $0.45 \tau$, f) $0.53 \tau$. $Q$ iso-surfaces $Q=0.1 u_{0}^{2} / d^{2}$ are highlighted in clear gray and black. A $2 \mathrm{C}$ vortex shedding pattern is observed in the upper section of the cylinder. 
be observed in Fig. 11 for $u_{r}^{*}=3.5, m_{r}^{*}=5$ (an intermediate amplitude case) and Fig. 12 for $u_{r}^{*}=5, m_{r}^{*}=7$ (a large amplitude case). Noticeable differences in the vortex shedding along the cylinder axis exist between these two cases. First, the phase difference along the cylinder span in the vortex-shedding is more pronounced in the large amplitude case than in the moderate amplitude one. Second, a recirculation bubble is formed near the cylinder surface in the middle and upper section of the cylinder. In the intermediate amplitude case, this bubble is quickly dissipated, while in the large amplitude case the bubble is shed and merges into the principal vortex of the shedding cycle. Plane cuts perpendicular to the axis of the cylinder at 2, 5 and 8 length units from the fixed point are done, showing values of $z$-vorticity together with Q-criterion iso-surfaces from a top view. A detailed description of the main characteristics of each case follows.

In the intermediate amplitude case, $u_{r}^{*}=3.5, m_{r}^{*}=5$, a $2 \mathrm{~S}$ (two single vortices shed per oscillation cycle) mode is observed in the lower section of the cylinder, (Figs. 11a, 11d, 11g, $11 \mathrm{j}$ and $11 \mathrm{~m})$. In the middle section of the cylinder a recirculation bubble with positive $z$-vorticity values enclosed by a large negative $z$-vorticity recirculation region located near the cylinder surface is formed Fig. 11b, at the same time a large recirculation region with positive $z$-vorticity develops in the other side of the cylinder (lower end in the Figures), this last vortex is the main recirculation region of this half oscillation cycle. The bubble evolves Fig. 11e and eventually detaches from the cylinder Fig. 11h but dissipates very soon without merging to the main structure Fig. 11k. In the upper section of the mast, a similar behavior is obtained, the recirculation bubble and the main vortex of the half cycle are created and develop Fig. 11c, 11f. The bubble and the main vortex are detached from the cylinder surface Fig. 11i, but again the bubble is dissipated quickly and is not merged to the main vortex Fig. 11l, which develops and is convected downstream Fig. 11o. Thus, a $2 \mathrm{~S}$ mode is observed along the complete length of the mastil near the cylinder surface.

In the large amplitude case, a $2 \mathrm{~S}$ mode is also observed in the lower section of the cylinder Figs. $12 \mathrm{a}, 12 \mathrm{~d}, 12 \mathrm{~g}, 12 \mathrm{j}$ and $12 \mathrm{~m}$. In the middle section, a very interesting vortex shedding pattern occurs: As before, the recirculation bubble with positive $z$-vorticity values is formed as the main recirculation region of this half cycle develops Fig. 12b. The bubble and the main vortex get connected, grow in extension Fig. 12e and eventually detach from the cylinder surface Fig. 12h. Both vortices are convected downstream Fig. 12k and merge into a single large counter-clockwise rotating vortex Fig. 12n. In the upper section of the mast, a very 
similar shedding pattern is observed with larger recirculation regions and vorticity values. Also, and making more evident that the shedding is out of phase along the cylinder, it can be observed that the bubble and the positive main vortex are detached from the cylinder Fig. 12f, merged Fig. 12i, and develop to a large recirculation region Fig. 12l, at earlier stages of the oscillation cycle. This vortex shedding pattern recalls the reported $2 \mathrm{~T}$ mode ${ }^{5}$, where two triads of vortices are shed per oscillation cycle. However, in the present case, the vortices are merged so close to the cylinder's surface that the characteristic print in the wake is absent. It is the authors' conjecture that considerably higher Reynolds numbers would make the vortices grow in strength and size giving place to the usual $2 \mathrm{~T}$ mode. However, further studies at larger $R e$ to confirm this feature are needed.

To conclude this section, the phase difference in the shedding process along the cylinder axis discussed previously can be quantified. To this end, the correlation functions of the $y^{*}$-component of the velocity very near the back surface of the cylinder at the heights shown in the plane cuts of Figs. 11, and 12 were calculated. In one hand, for the intermediate amplitude case $\left(u_{r}^{*}=3.5, m_{r}^{*}=5\right)$, the maximum phase shift obtained between the upper and lower section of the cylinder is $0.012 \pi$. The shedding process is almost done in phase for this case. In the other hand, noticeable phase shifts for the large amplitude case $\left(u_{r}^{*}=5\right.$, $m_{r}^{*}=7$ ) are obtained: $0.042 \pi$ between the upper and the middle section, and $0.063 \pi$ between the upper and the lower section of the cylinder.

\section{ANALYTICAL APPROXIMATIONS}

In order to gain physical insight and understanding of the cylinder displacement, analytical approximations to the solutions of its equations of motion can be derived. The phase shift between forces and displacements will be discussed, and it will be shown that, for the explored parameters, the system does not reach a resonant state. Moreover, the analytical approximations can be used in the future in a reduced order model for the present VIV system.

Let $\theta=\bar{\theta}-\varepsilon \Theta$ and $\phi=-\varepsilon \Phi$, where $\varepsilon$ is an expansion parameter, the nondimensional equations up to terms of order $\varepsilon$ can be obtained directly from eqs. 17. The zeroth order equation is 


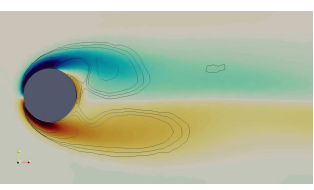

(a)

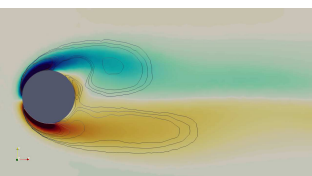

(d)

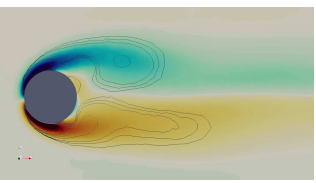

(g)

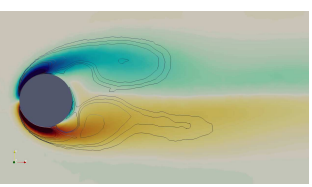

(j)

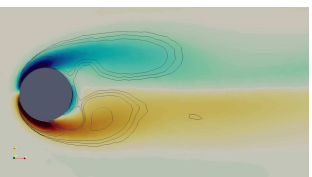

(m)

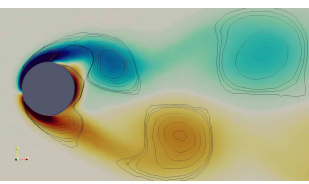

(b)

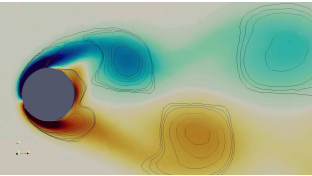

(e)

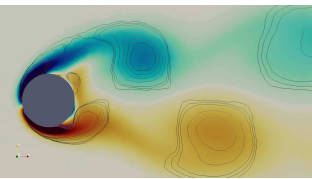

(h)

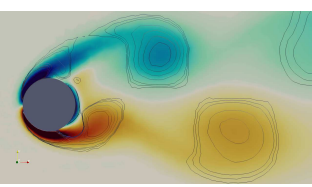

$(\mathrm{k})$

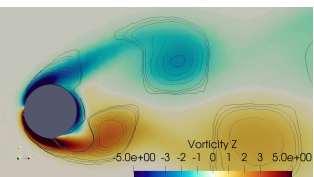

(n)

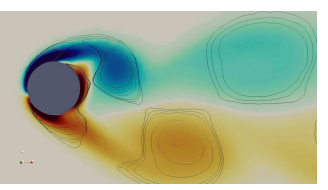

(c)

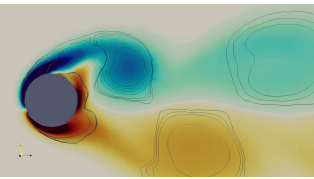

(f)

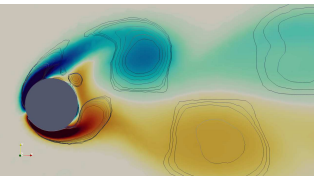

(i)

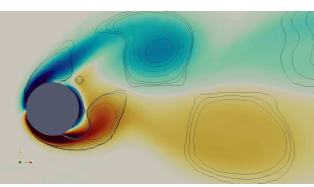

(1)

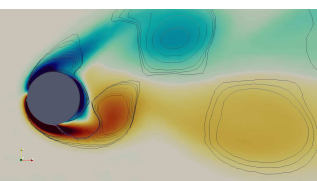

(o)

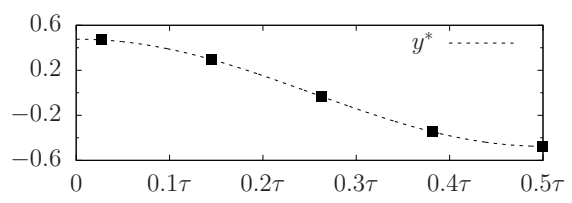

FIG. 11: Vortex shedding pattern along the cylinder for $u_{r}^{*}=3.5, m_{r}^{*}=5$. Plane cuts are taken perpendicular to the cylinder axis at 2 (left), 5 (center) and 8 (right) length units from the pivot. The rows correspond to $0.02 \tau$ (a, b, c), $0.15 \tau$ (d, e, f), $0.26 \tau$ (g, h, i), $0.38 \tau$ $(\mathrm{j}, \mathrm{k}, \mathrm{l})$ and $0.5 \tau(\mathrm{m}, \mathrm{n}, \mathrm{o})$. 


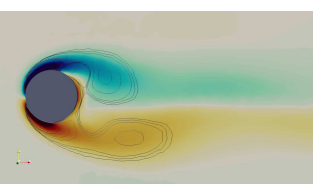

(a)

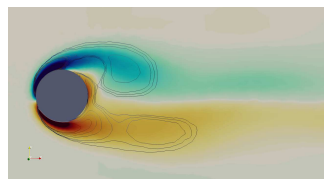

(d)

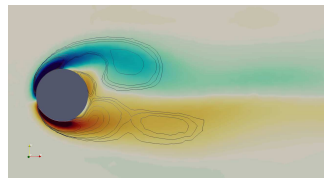

(g)

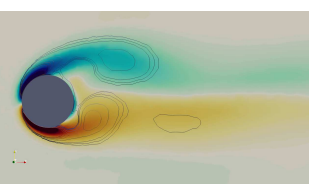

(j)

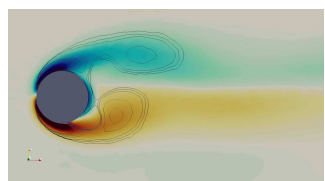

(m)

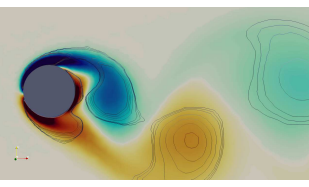

(b)

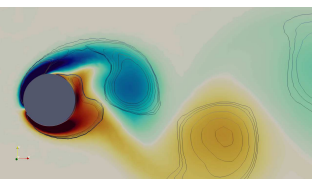

(e)

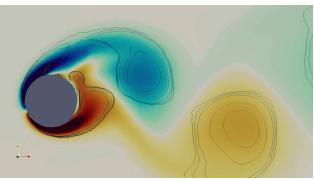

(h)

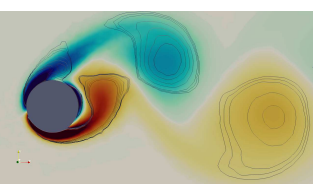

$(\mathrm{k})$

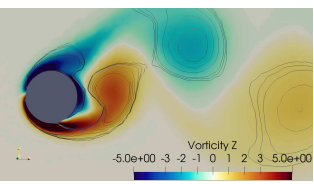

(n)

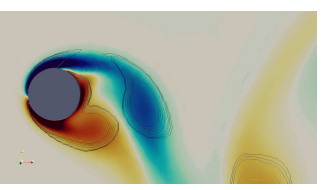

(c)

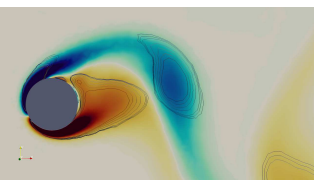

(f)

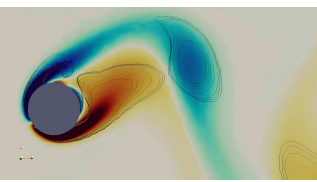

(i)

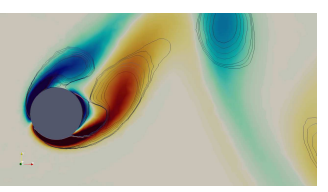

(1)

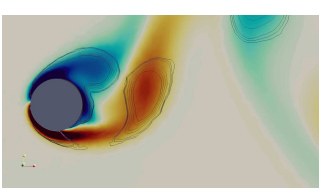

(o)

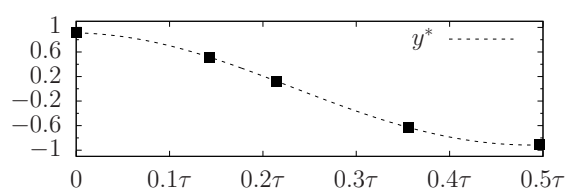

FIG. 12: Vortex shedding pattern along the cylinder for $u_{r}^{*}=5, m_{r}^{*}=7$. Plane cuts are taken perpendicular to the cylinder axis at 2 (left), 5 (center) and 8 (right) length units from the pivot. The rows correspond to 0 (a, b, c), $0.15 \tau(\mathrm{d}, \mathrm{e}, \mathrm{f}), 0.21 \tau(\mathrm{g}, \mathrm{h}, \mathrm{i}), 0.36 \tau(\mathrm{j}$, $\mathrm{k}, \mathrm{l})$ and $0.5 \tau(\mathrm{m}, \mathrm{n}, \mathrm{o})$. 


$$
\frac{\cos \bar{\theta}}{m_{g r}^{*}}-\omega_{0}^{2}[\sin \bar{\theta} \cos \bar{\theta}]-\frac{2}{\pi m_{r}^{*}}\left[\bar{C}_{z} \cos \bar{\theta}-\bar{C}_{x} \sin \bar{\theta}\right]=0,
$$

where $\omega_{0}$ is the nondimensional natural frequency, $\omega_{0}=2 \pi / u_{r}^{*}$ and $\widetilde{C}_{i}$ denote the first order force coefficient fluctuations in the $i$ direction.

An excellent approximation for $\bar{\theta}$ can be obtained and is

$$
\bar{\theta} \simeq \cos ^{-1}\left[\frac{S \bar{C}_{x}}{1-S_{g}+S \bar{C}_{z}}\right]
$$

where $S=\frac{2}{\omega_{0}^{2} \pi m_{r}^{*}}=\frac{u_{r}^{* 2}}{2 \pi^{3} m_{r}^{*}}$ is related to the spring stiffness and $S_{g}=\frac{1}{\omega_{0}^{2} m_{g r}^{*}}=\frac{u_{r}^{* 2}}{(2 \pi)^{2} m_{g r}^{*}}$ relates the weight of the cylinder to the aerodynamic force.

Due to the fact that the value of $\bar{C}_{z}$ is negative, the second and the third terms in the denominator of Eq. (26) tend to decrease the value of the cylinder angle. In this work it is assumed that the influence of the cylinder weight is negligible and the value of $S_{g}<<1$. In this case, the average angle of the cylinder can be given by $\bar{\theta} \simeq \cos ^{-1}\left(S \bar{C}_{x}\right)$ for the cases where $\bar{C}_{z}$ is small compared with unity; the values of $\bar{C}_{x}$ are of order unity and are a function of the Reynolds number and the parameters $m_{r}^{*}$ and $u_{r}^{*}$ (see Table II). Then the zeroth order solution for $\bar{\theta}$ can be obtained and is shown in Fig. 13 compared with the numerical values obtained in this work. It is pointed out that both solutions match quite well. Small deviations are observed at large reduced velocity values, where relatively large magnitude of $\bar{C}_{z}$ was obtained, although the maximum differences are about $4 \%$.

The first order equations in $\varepsilon$ are

$$
\begin{aligned}
& \ddot{\Theta}+\omega_{0}^{2} \Theta\left[\left(2 \sin ^{2} \bar{\theta}-1\right)-S_{g} \sin \bar{\theta}+S\left(\bar{C}_{z} \sin \bar{\theta}+\bar{C}_{x} \cos \bar{\theta}\right)\right]+ \\
& \beta_{x} \dot{\Theta}=\frac{2}{\pi m_{r}^{*}}\left(\widetilde{C}_{x} \sin \bar{\theta}-\widetilde{C}_{z} \cos \bar{\theta}\right) \equiv \widetilde{F}_{x} \\
& \ddot{\Phi}+\omega_{0}^{2} \Phi\left[1-\frac{S_{g}}{\sin \bar{\theta}}+\frac{S \bar{C}_{z}}{\sin ^{2} \bar{\theta}}\right]+\beta_{y} \dot{\Phi}=\frac{2 \widetilde{C}_{y}}{\pi m_{r}^{*} \sin ^{2} \bar{\theta}} \equiv \widetilde{F}_{y},
\end{aligned}
$$

where $\beta_{x}=4 \pi \zeta_{\theta}^{*} / u_{r}^{*}$ and $\beta_{y}=4 \pi \zeta_{\phi}^{*} /\left(u_{r}^{*} \sin ^{2} \bar{\theta}\right)$ are the damping parameters in the $x^{*}$ and $y^{*}$ directions respectively, while $\widetilde{F}_{x}$ and $\widetilde{F}_{y}$ are the corresponding force fluctuations. The natural frequency depends on the average cylinder inclination and is not affected by gravity for very small values of the parameter $S_{g}$ compared to unity. Taking into account that the values of the parameter $S$ are small compared with unity for the cases considered in the present work, an excellent approximation can be obtained by assuming the value of unity 


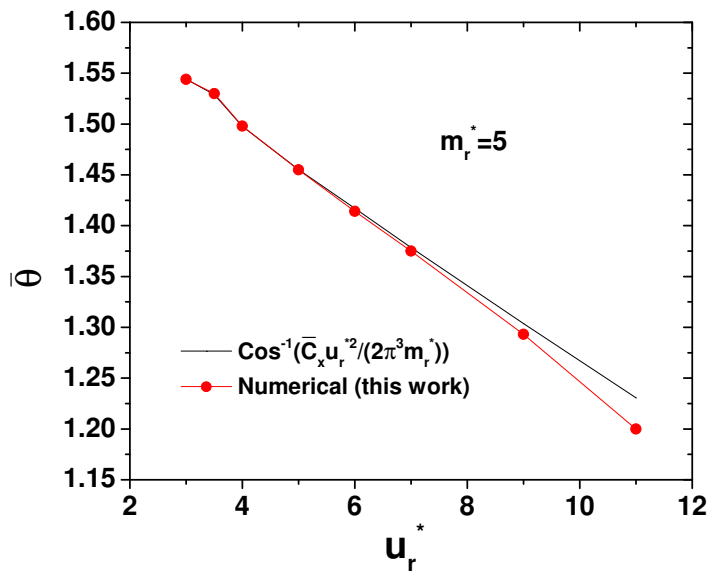

FIG. 13: Zeroth order solution of the average cylinder angle compared with the numerical result.

inside the square brackets on the left-hand sides of 27. The equations of a classical drivendamped oscillator are then obtained and the amplitude of the angular fluctuations $\Theta_{m}$ and $\Phi_{m}$ are related to the amplitude of the forcing fluctuations $F_{x m}$ and $F_{y m}$ as

$$
\Theta_{m}=\frac{F_{x m}}{\sqrt{\left(\omega_{0}^{2}-\omega_{x}^{2}\right)^{2}+\omega_{x}^{2} \beta_{x}^{2}}}
$$

and

$$
\Phi_{m}=\frac{F_{y m}}{\sqrt{\left(\omega_{0}^{2}-\omega_{y}^{2}\right)^{2}+\omega_{y}^{2} \beta_{y}^{2}}},
$$

where the respective forcing frequencies are $\omega_{x}=2 \omega_{y}$. It is pointed out, that the main assumption deriving the previous equations is the small value of the $\bar{C}_{z}$ force coefficient. This assumption will not remain valid if large inclination angles are obtained, which are related to small values of the reduced mass, or large values of the reduced velocity. The phase lag between the forcing and the displacement $\sigma$ can be obtained for each direction as

$$
\sigma=\tan ^{-1}\left(-\frac{\omega \beta}{\omega_{0}^{2}-\omega^{2}}\right) .
$$

It can be observed that $-\pi<\sigma<0$. Fig. 14 shows the values of the non-dimensional oscillation frequencies $\omega / 2 \pi$ together with the nondimensional natural frequency $\omega_{0} / 2 \pi$ for 


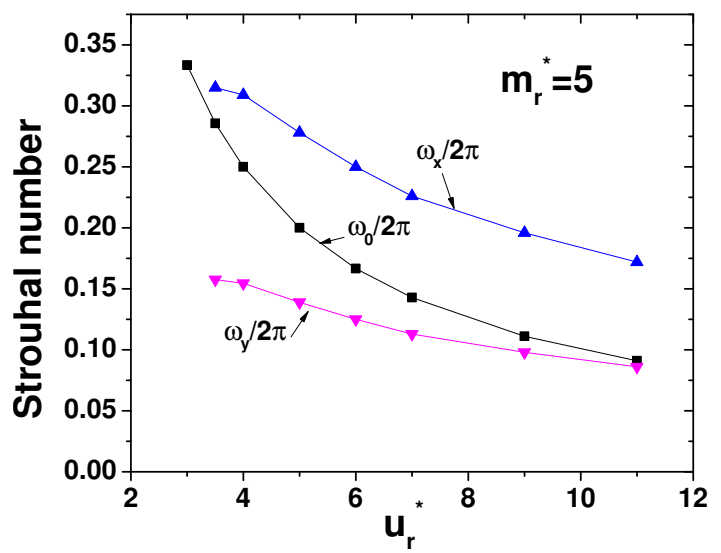

FIG. 14: Nondimensional frequencies resulting from the parametric set.

the different set of parameters considered. In all the studied cases in this work, the natural frequency lies between both forcing frequencies, that is $\omega_{0}>\omega_{y}$ and $\omega_{0}<\omega_{x}$. Therefore, on the one hand, $\sigma_{x}<-\pi / 2$ and given that the damping parameter is assumed to be small compared with unity $\sigma_{x} \simeq-\pi$. On the other hand $\sigma_{y}>-\pi / 2$, and for small values of the damping parameter it implies $\sigma_{y} \simeq 0$. Moreover, it can be concluded that the system is never in resonance and it is natural to suppose that reaching a resonance region in the $y^{*}$ direction would give an extra amplification in the oscillation amplitude of the cylinder. Further research in the parameter space is needed.

The fluctuations of the cylinder tip in both coordinates are then

$$
\Delta x^{*}=10.5 \varepsilon \sin \bar{\theta} \Theta_{m} ; \Delta y^{*}=10.5 \varepsilon \Phi_{m}
$$

The amplitude of the cylinder tip displacement in the $y^{*}$-direction in the numerical experiments is known for a value of $m_{r}^{*}=5$ and different values of $u_{r}^{*}$ (see Table I). From these data, the maximum angular deviations are obtained as $\phi_{m}=\sin ^{-1}\left(\Delta y^{*} / 10.5\right)$ and can be compared with the analytical approximation. This comparison is plotted in Fig. 15, with $\Phi_{m}$ given by Eq.(29). It is pointed out that due to the presence of the expansion parameter $\varepsilon$, the absolute value differences observed are not relevant. The important fact is that both solutions follow the same trend with only slight deviations at large values of $u_{r}^{*}$. 


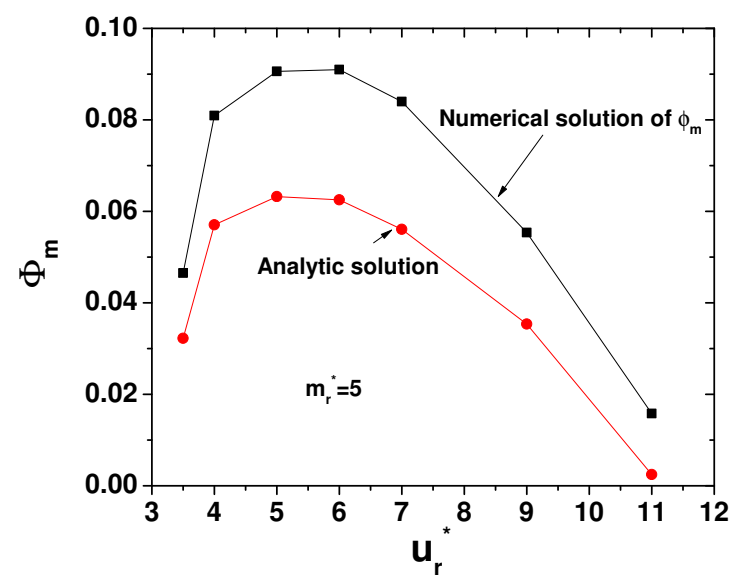

FIG. 15: Numerical and analytical transverse angular fluctuations for different values of $u_{r}^{*}$ with a fixed value of $m_{r}^{*}=5$.

\section{CONCLUSIONS AND FUTURE WORK}

A three-dimensional FSI model for VIV of a pivoted cylinder was introduced and mathematically described. The governing equations were solved numerically for fixed low Reynolds numbers $R e=100,200$ and different values of the relevant non-dimensional parameters, the reduced velocity $u_{r}^{*}$, the reduced mass $m_{r}^{*}$ and a fixed non-zero value of the non-dimensional damping parameter. The results show that the mathematical and numerical model can reproduce the cylinder dynamical response and the vortex structure of the flow reported in experimental studies of similar systems at larger $R e$.

The cylinder displacement in the cross-flow direction as a function of the reduced velocity $u_{r}^{*}$ can be separated in a three branches response, as reported in experimental works for elastically mounted ${ }^{5}$ and pivoted cylinders ${ }^{7}$. In the in-line direction, small amplitudes around the inclined axis of the cylinder are observed. Small values of the reduced mass and large values of the reduced velocity produce large deviations from the vertical axis of the cylinder. The comparison with the elastically mounted cases in the laminar regime ${ }^{17,18}$ suggest that the pivoted configuration has a wider excitation region. However, more research in the $I$ and $U$ branches is needed to draw definitive conclusion. 
The hydrodynamic force coefficients were calculated and their response described in detail. The fixed and quasi-static cases have a mean drag coefficient $\bar{C}_{x}$ that compares well with the one obtained for a two free-ends fixed cylinder case ${ }^{8}$. Interesting relations between the hydrodynamic force coefficients and the inclination angle of the mast, typical of this pivoted configuration, were found (see Sec.IVB). The wake structure show that the vortex shedding is done out of phase along the cylinder span for cases with large amplitudes. Provoking vortex dislocations near the upper and lower sections of the cylinder. The $2 \mathrm{C}$ vortex formation pattern has been reproduced and its connection with the vortex dislocations and the merging of the coherent structures shed from opposite sides of the cylinder has been shown. A singular structure in the wake, caused by a reversed flow behind the cylinder is observed for the case $u_{r}^{*}=5, m_{r}^{*}=7$. Details of the vortex-shedding process close to the cylinder surface shows the formation and separation of an additional vortical structure per half oscillation cycle for different amplitude cases. It is the authors' conjecture that this could be the onset of the $2 \mathrm{~T}$ vortex-shedding pattern reported at higher Reynolds numbers. However, no evidence of this mode in the wake is obtained as the extra vortical structure dissipates or merges quickly with the main vortex in the considered cases. Thus, higher Reynolds number studies of this system are proposed to shed light on this particular aspect.

The analytical approximation for the solution of the cylinder equations of motion, presented in Sec. V, show excellent agreement with the numerical results. It also shows that the phase lag between the forcing and the displacement is $\sigma_{x} \simeq-\pi$ in the $x^{*}$-direction and $\sigma_{y} \simeq 0$ in the $y^{*}$-direction given the small value of the damping parameter used in the present study. Also, it is observed that for the set of parameters used, the natural frequency of the system lies between the forcing frequencies in the $x^{*}$ and $y^{*}$ directions. Thus, the system is never in resonance. It is observed that reaching a resonance region in the $y^{*}$ direction would finally give an extra amplification in the oscillations of the system. Future research will be directed to this end.

Finally, it is pointed out that not only the results of the simulations are valuable; the mathematical model itself can be extremely useful. The presented model is complex enough to capture the main physical characteristics of VIV in circular pivoted cylinders at the same time that it is simple enough (thanks to the solid model and the low Reynolds number) to make possible the use of analytical approximations and the use of stability analysis techniques in the future. To mention two examples, studying this system from the numerical 
stability point of view can lead to develop physics-based algorithms for general FSI problems; and also the obtained results can be used as base flow to analyze the stability of the trajectories followed by the cylinder in the VIV process.

\section{ACKNOWLEDGMENTS}

This work has been partially financially supported by the 'Ministerio de Economía y Competitividad, Secretaría de Estado de Investigación, Desarrollo e Innovacion', Spain (Ref.TRA2017-88508-R). D. Pastrana acknowledges support of the CONACyT-SENER graduate fellowship program to study abroad 278102/439162. J.C. Cajas acknowledges the financial support of UNAM through the project PAPIIT-IA106120 and the access to supercomputing resources at Miztli: LANCAD-UNAM-DGTIC-379.

\section{AIP DATA SHARING POLICY}

The data that support the findings of this study are available from the corresponding author upon reasonable request.

\section{REFERENCES}

${ }^{1} \mathrm{~T}$. Sarpkaya, "A critical review of the intrinsic nature of vortex-induced vibrations," Journal of Fluids and Structures 19, 389-447 (2004).

${ }^{2}$ C. H. K. Williamson and R. Govardhan, "A brief review of recent results in vortexinduced vibrations," Journal of Wind Engineering and Industrial Aerodynamics 96, 713735 (2008).

${ }^{3}$ P. W. Bearman, "Circular cylinder wakes and vortex-induced vibrations," Journal of Fluids and Structures 27, 648-658 (2011).

${ }^{4}$ A. Khalak and C. H. K. Williamson, "Fluid Forces And Dynamics Of A Hydroelastic Structure With Very Low Mass And Damping," Journal of Fluids and Structures 11, 973-982 (1997).

${ }^{5}$ N. Jauvtis and C. H. K. Williamson, "The effect of two degrees of freedom on vortexinduced vibration at low mass and damping," Journal of Fluid Mechanics 509, 23-62 (2004). 
${ }^{6}$ C. H. K. Williamson, "Oblique and parallel modes of vortex shedding in the wake of a circular cylinder at low Reynolds numbers," Journal of Fluid Mechanics 206, 579?627 (1989).

${ }^{7}$ F. Flemming and C. H. K. Williamson, "Vortex-induced vibrations of a pivoted cylinder," Journal of Fluid Mechanics 522, 215-252 (2005).

${ }^{8}$ O. Inoue and A. Sakuragi, "Vortex shedding from a circular cylinder of finite length at low Reynolds numbers," Physics of Fluids 20, 033601 (2008).

${ }^{9}$ E. Marble, C. Morton, and S. Yarusevych, "Spanwise wake development of a pivoted cylinder undergoing vortex-induced vibrations with elliptic trajectories," Experiments in Fluids 60, 81 (2019).

${ }^{10}$ D. Pastrana, J. Cajas, O. Lehmkuhl, I. Rodríguez, and G. Houzeaux, "Large-eddy simulations of the vortex-induced vibration of a low mass ratio two-degree-of-freedom circular cylinder at subcritical Reynolds numbers," Computers \& Fluids 173, 118-132 (2018).

${ }^{11}$ Y. Bazilevs, K. Takizawa, and T. E. Tezduyar, Front Matter, in Computational FluidStructure Interaction: Methods and Applications (John Wiley \& Sons, Ltd, Chichester, UK, 2013).

${ }^{12}$ T. Richter, Fluid-structure Interactions, Lecture Notes in Computational Science and Engineering, Vol. 118 (Springer International Publishing, 2017).

${ }^{13}$ S. Singh and S. Mittal, "Vortex-induced oscillations at low Reynolds numbers: Hysteresis and vortex-shedding modes," Journal of Fluids and Structures 20, 1085 - 1104 (2005).

${ }^{14}$ T. K. Prasanth and S. Mittal, "Vortex-induced vibrations of a circular cylinder at low Reynolds numbers," Journal of Fluid Mechanics (2008), 10.1017/S0022112007009202.

${ }^{15}$ T. Prasanth and S. Mittal, "Vortex-induced vibration of two circular cylinders at low Reynolds number," Journal of Fluids and Structures 25, 731 - 741 (2009), bluff Body Wakes and Vortex-Induced Vibrations (BBVIV-5).

${ }^{16}$ T. K. Prasanth, V. Premchandran, and S. Mittal, "Hysteresis in vortex-induced vibrations: critical blockage and effect of m*," Journal of Fluid Mechanics 671, 207 ?225 (2011).

${ }^{17}$ R. Bourguet and D. Lo Jacono, "Flow-induced vibrations of a rotating cylinder," Journal of Fluid Mechanics 740, 342-380 (2014).

${ }^{18} \mathrm{M}$. Zhao, L. Cheng, and L. Lu, "Vortex induced vibrations of a rotating circular cylinder at low Reynolds number," Physics of Fluids 26, 073602 (2014). 
${ }^{19}$ D. Lo Jacono, R. Bourguet, M. C. Thompson, and J. S. Leontini, "Three-dimensional mode selection of the flow past a rotating and inline oscillating cylinder," Journal of Fluid Mechanics 855, 1-11 (2018).

${ }^{20} \mathrm{R}$. Bourguet, "Two-degree-of-freedom flow-induced vibrations of a rotating cylinder," Journal of Fluid Mechanics (2020), 10.1017/jfm.2020.403.

${ }^{21}$ P. Han, P. Hémon, G. Pan, and E. de Langre, "Nonlinear modeling of combined galloping and vortex-induced vibration of square sections under flow," Nonlinear Dynamics 103, 3113-3125 (2021).

${ }^{22} \mathrm{~S}$. Gsell, R. Bourguet, and M. Braza, "Two-degree-of-freedom vortex-induced vibrations of a circular cylinder at Re=3900," Journal of Fluids and Structures 67, 156-172 (2016).

${ }^{23}$ A. Fujarra, C. Pesce, F. Flemming, and C. Williamson, "Vortex-induced vibration of a flexible cantilever," Journal of Fluids and Structures 15, 651 - 658 (2001).

${ }^{24}$ G. Palau-Salvador, T. Stoesser, J. Fröhlich, M. Kappler, and W. Rodi, "Large eddy simulations and experiments of flow around finite-height cylinders," Flow, Turbulence and Combustion 84, 239 (2009).

${ }^{25}$ P. Levold, Viscous Flow around finite length circular cylinder, Ph.D. thesis, Trondheim: Norwegian University of Science and Technology (Marine Techonology) (2014).

${ }^{26}$ J. R. Meneghini, F. Saltara, R. de Andrade Fregonesi, C. T. Yamamoto, E. Casaprima, and J. A. Ferrari, "Numerical simulations of viv on long flexible cylinders immersed in complex flow fields," European Journal of Mechanics - B/Fluids 23, 51 - 63 (2004), bluff Body Wakes and Vortex-Induced Vibrations.

${ }^{27}$ F. Xie, J. Deng, Q. Xiao, and Y. Zheng, "A numerical simulation of VIV on a flexible circular cylinder," Fluid Dynamics Research 44, 045508 (2012).

${ }^{28}$ J. Cajas, G. Houzeaux, M. Vázquez, M. García, E. Casoni, H. Calmet, A. Artigues, R. Borrell, O. Lehmkuhl, D. Pastrana, et al., "Fluid-structure interaction based on hpc multicode coupling," SIAM Journal on Scientific Computing 40, C677-C703 (2018).

${ }^{29}$ C. Farhat, M. Lesoinne, and P. LeTallec, "Load and motion transfer algorithms for fluid/structure interaction problems with non-matching discrete interfaces: Momentum and energy conservation, optimal discretization and application to aeroelasticity," Computer methods in applied mechanics and engineering 157, 95-114 (1998).

${ }^{30}$ U. Küttler and W. A. Wall, "Fixed-point fluid-structure interaction solvers with dynamic relaxation," Computational mechanics 43, 61-72 (2008). 
${ }^{31}$ W. Dettmer and D. Perić, "A computational framework for fluid-structure interaction: Finite element formulation and applications," Computer methods in applied mechanics and engineering 195, 5754-5779 (2006).

${ }^{32}$ T. E. Tezduyar, "Finite elements in fluids: Stabilized formulations and moving boundaries and interfaces," Computers \& fluids 36, 191-206 (2007).

${ }^{33}$ J. M. Dahl, F. S. Hover, M. S. Triantafyllou, S. Dong, and G. E. Karniadakis, "Resonant vibrations of bluff bodies cause multivortex shedding and high frequency forces," Physical Review Letters 99, 5-8 (2007).

${ }^{34} \mathrm{G}$. Houzeaux and J. Principe, "A variational subgrid scale model for transient incompressible flows," Int. J. CFD 22, 135-152 (2008).

${ }^{35}$ G. Houzeaux, R. Aubry, and M. Vázquez, "Extension of fractional step techniques for incompressible flows: The preconditioned orthomin(1) for the pressure schur complement," Computers \& Fluids 44, 297-313 (2011).

${ }^{36}$ G. Houzeaux, R. de la Cruz, H. Owen, and M. Vázquez, "Parallel uniform mesh multiplication applied to a Navier-Stokes solver," Computers \& Fluids 80, 142-151 (2013).

${ }^{37}$ Y. Liu, R. M. So, and Z. X. Cui, "A finite cantilevered cylinder in a cross-flow," Journal of Fluids and Structures 20, 589-609 (2005).

${ }^{38}$ R. Feynman, R. Leighton, and M. Sands, The Feynman lectures on physics (Reading, Mass. : Addison-Wesley Pub. Co., 1963).

${ }^{39} \mathrm{~S}$. Krajnović, "Flow around a tall finite cylinder explored by large eddy simulation," Journal of Fluid Mechanics 676, 294-317 (2011).

${ }^{40}$ O. Lehmkuhl, I. Rodríguez, R. Borrell, and A. Oliva, "Low-frequency unsteadiness in the vortex formation region of a circular cylinder," Physics of Fluids 25, 085109 (2013).

${ }^{41}$ D. Aljure, O. Lehmkhul, I. Rodríguez, and A. Oliva, "Three dimensionality in the wake of the flow around a circular cylinder at Reynolds number 5000," Computers \& Fluids 47, $102-118$ (2017). 

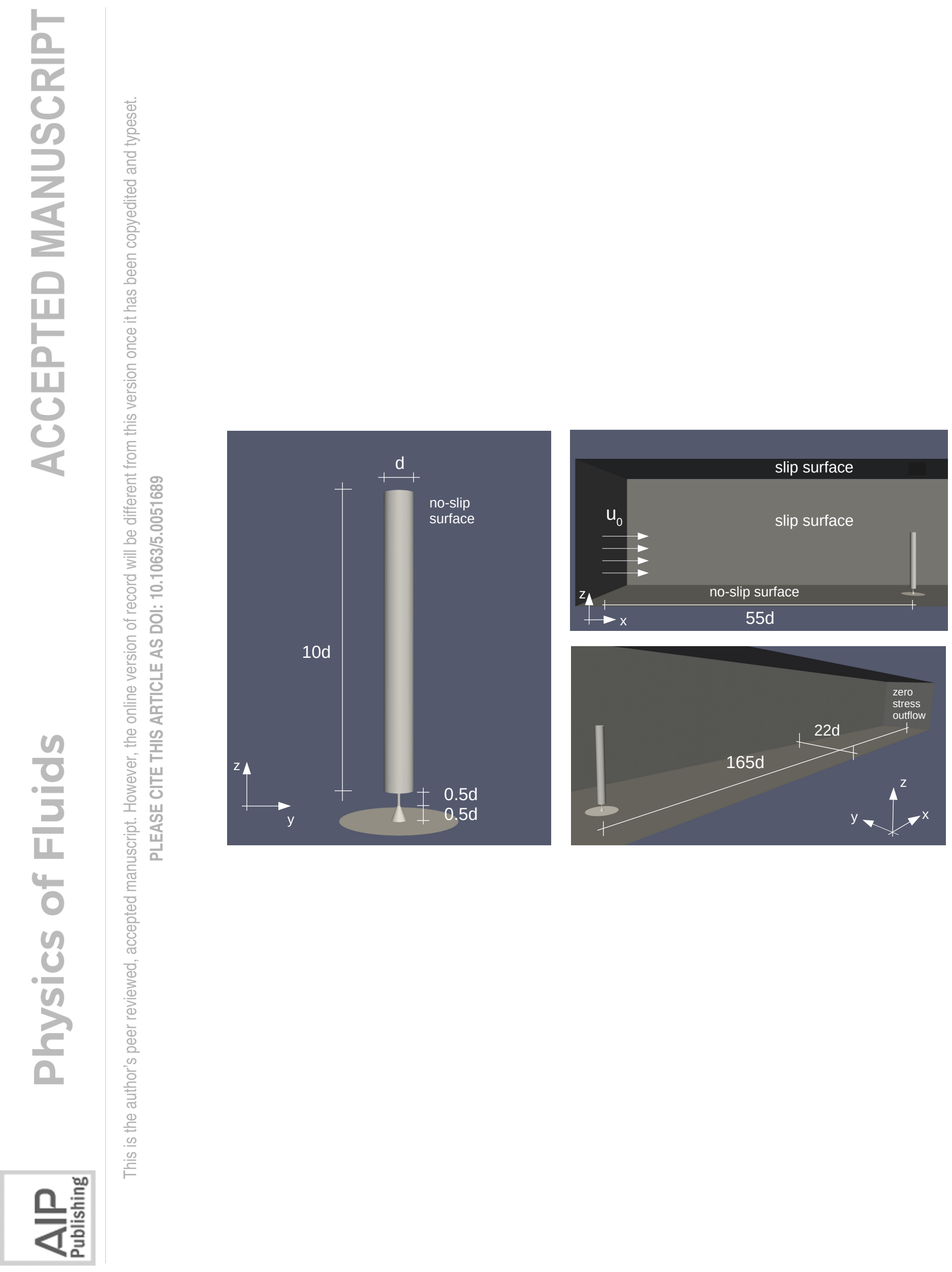

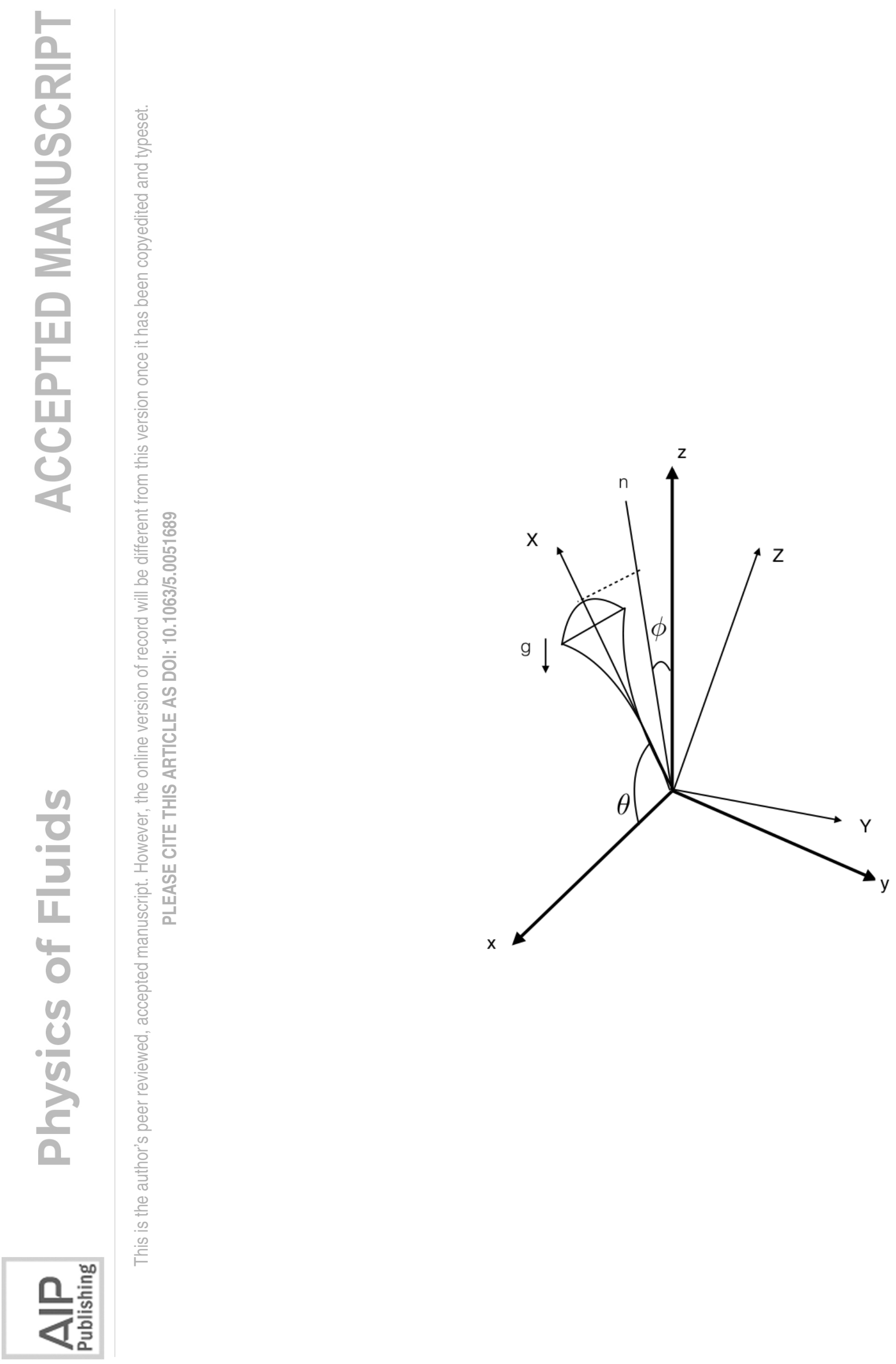


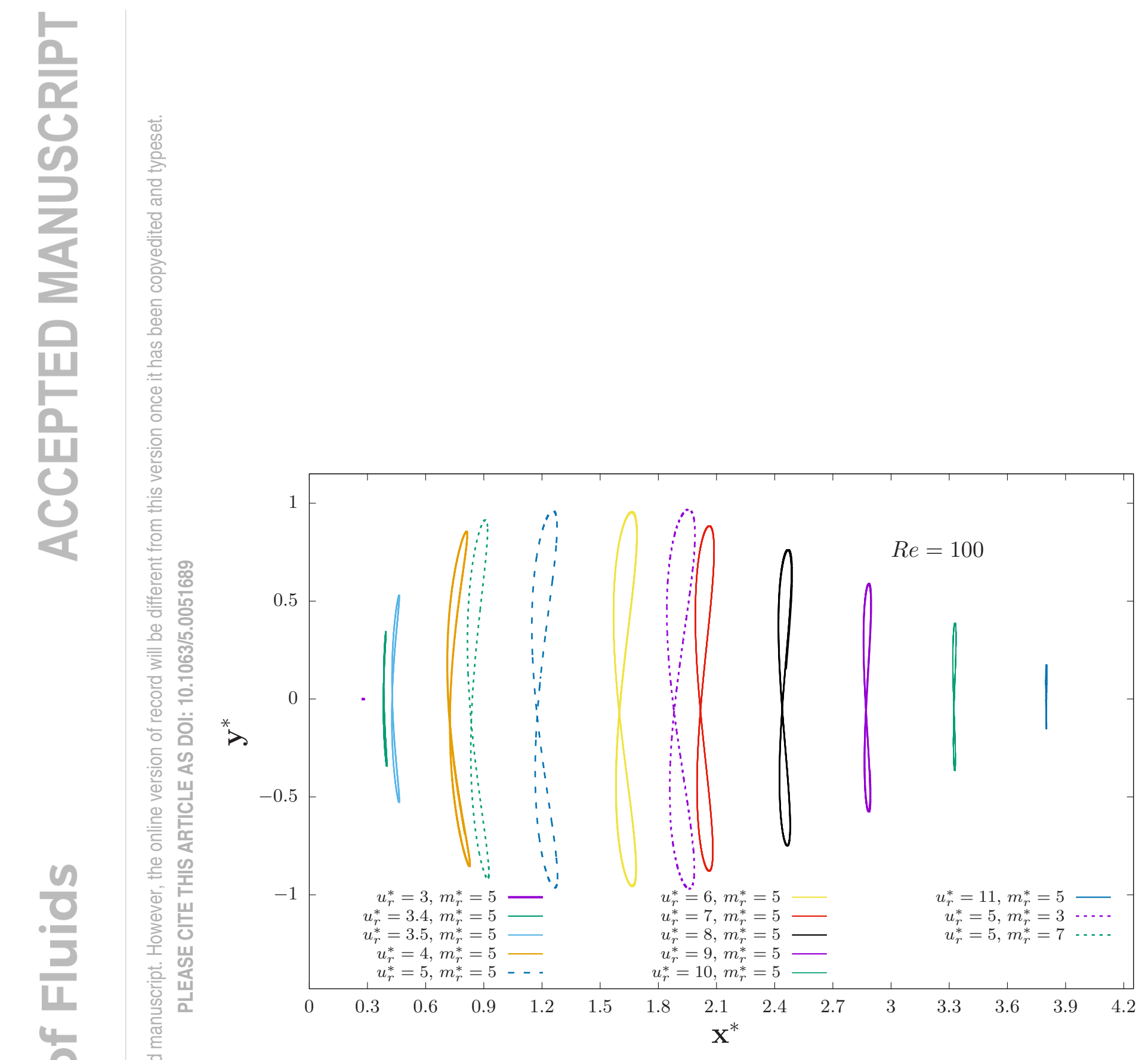




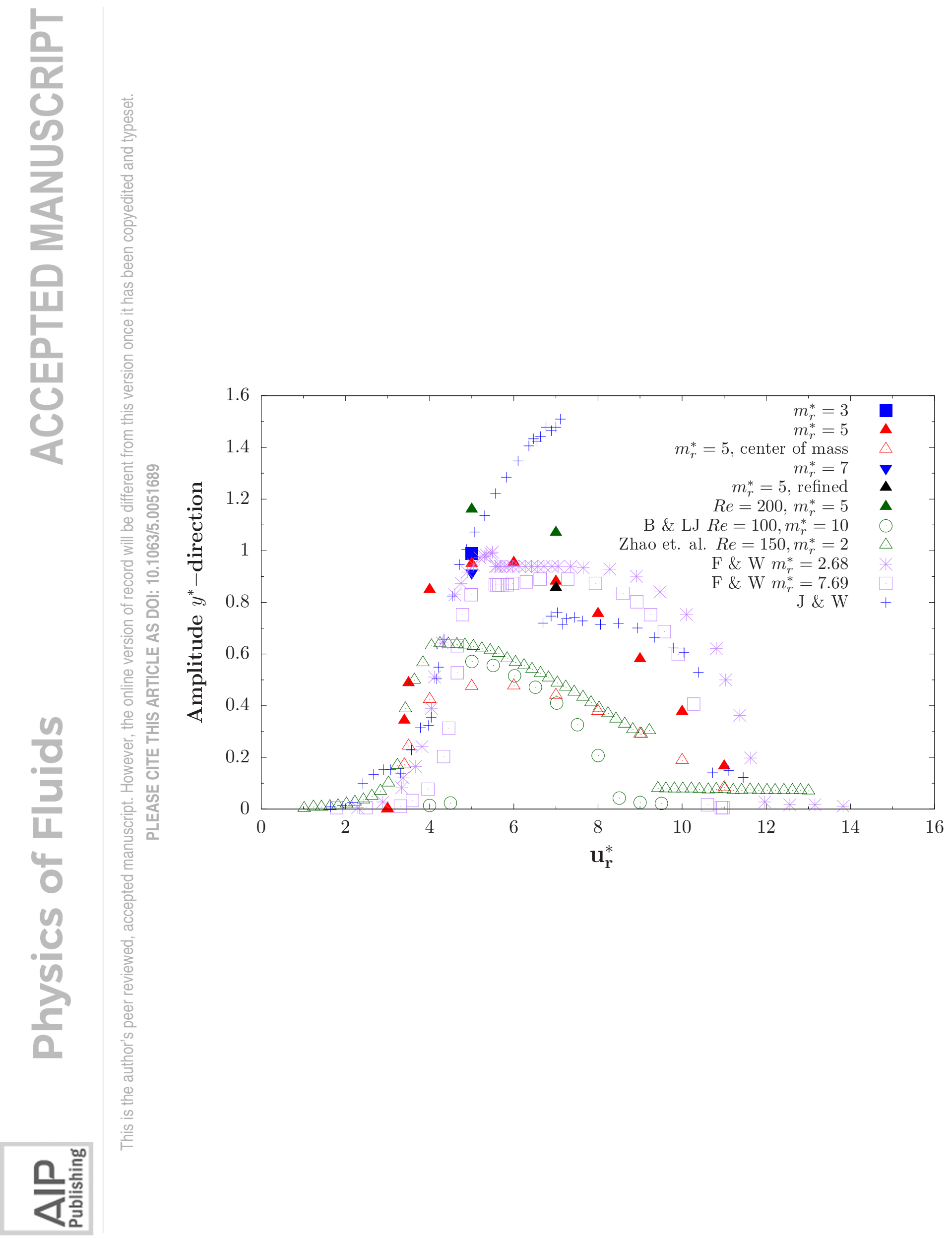




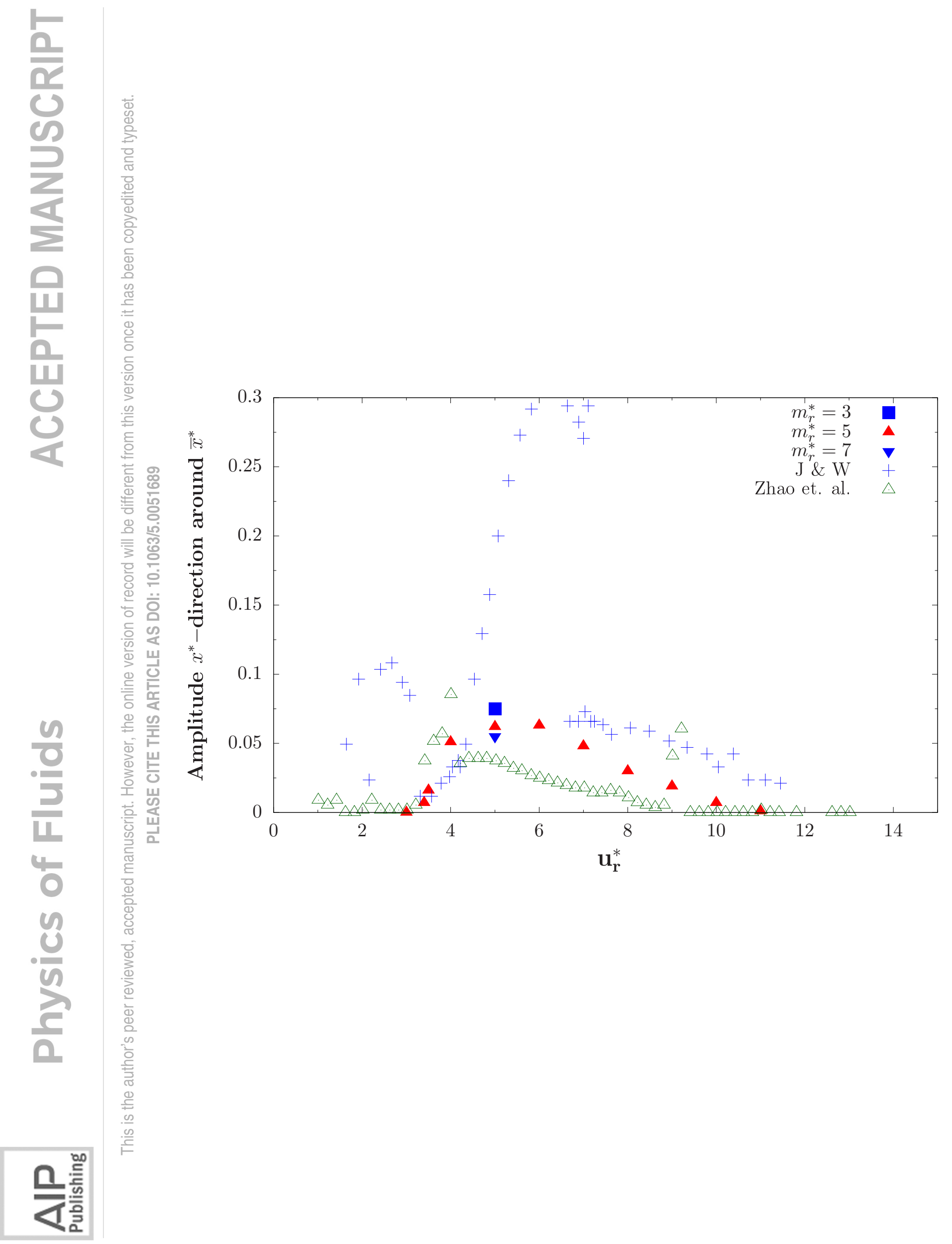




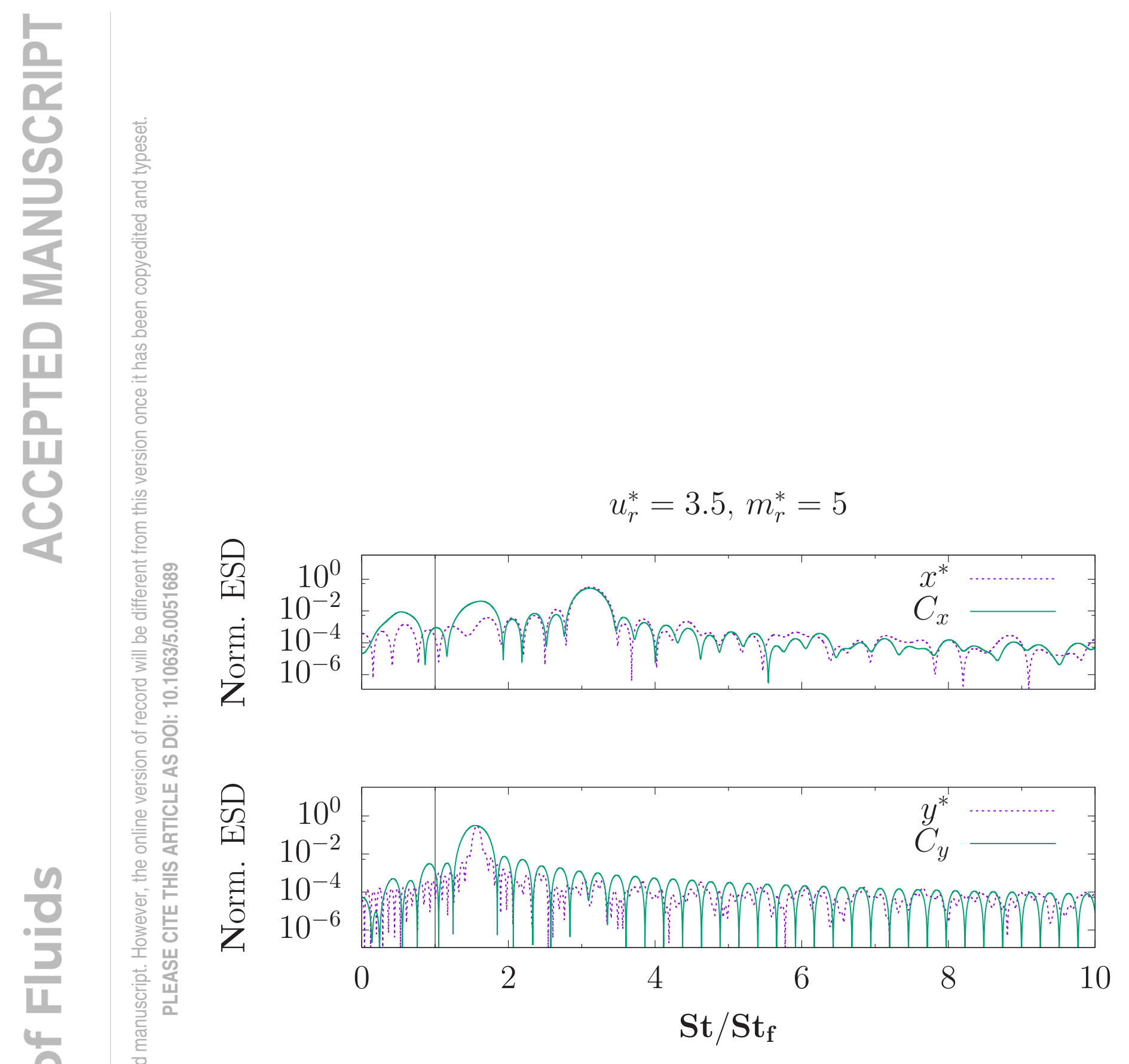




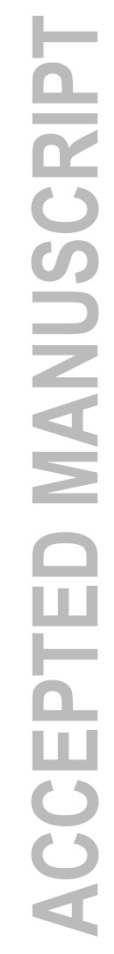

$$
u_{r}^{*}=5, m_{r}^{*}=3
$$
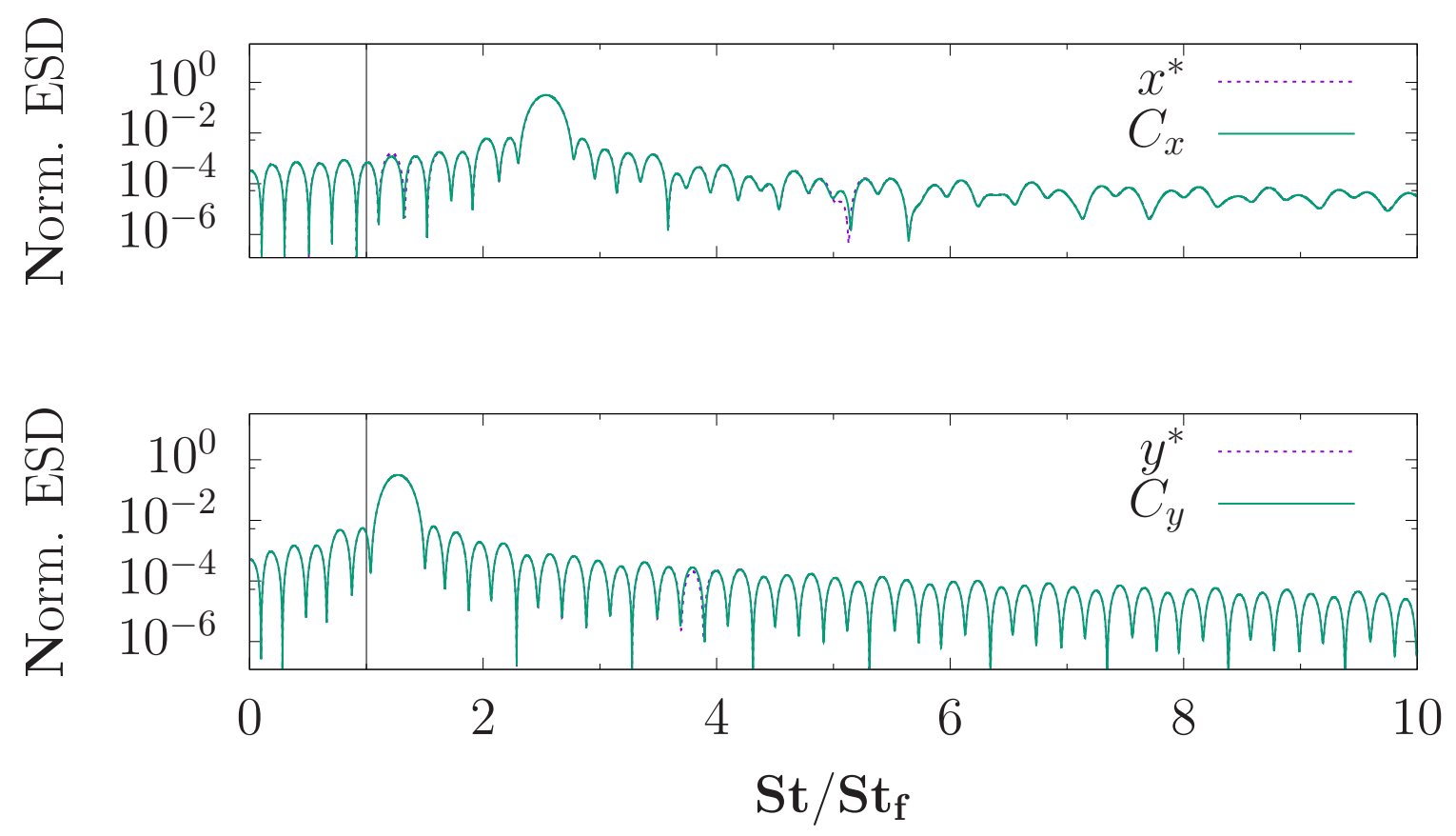


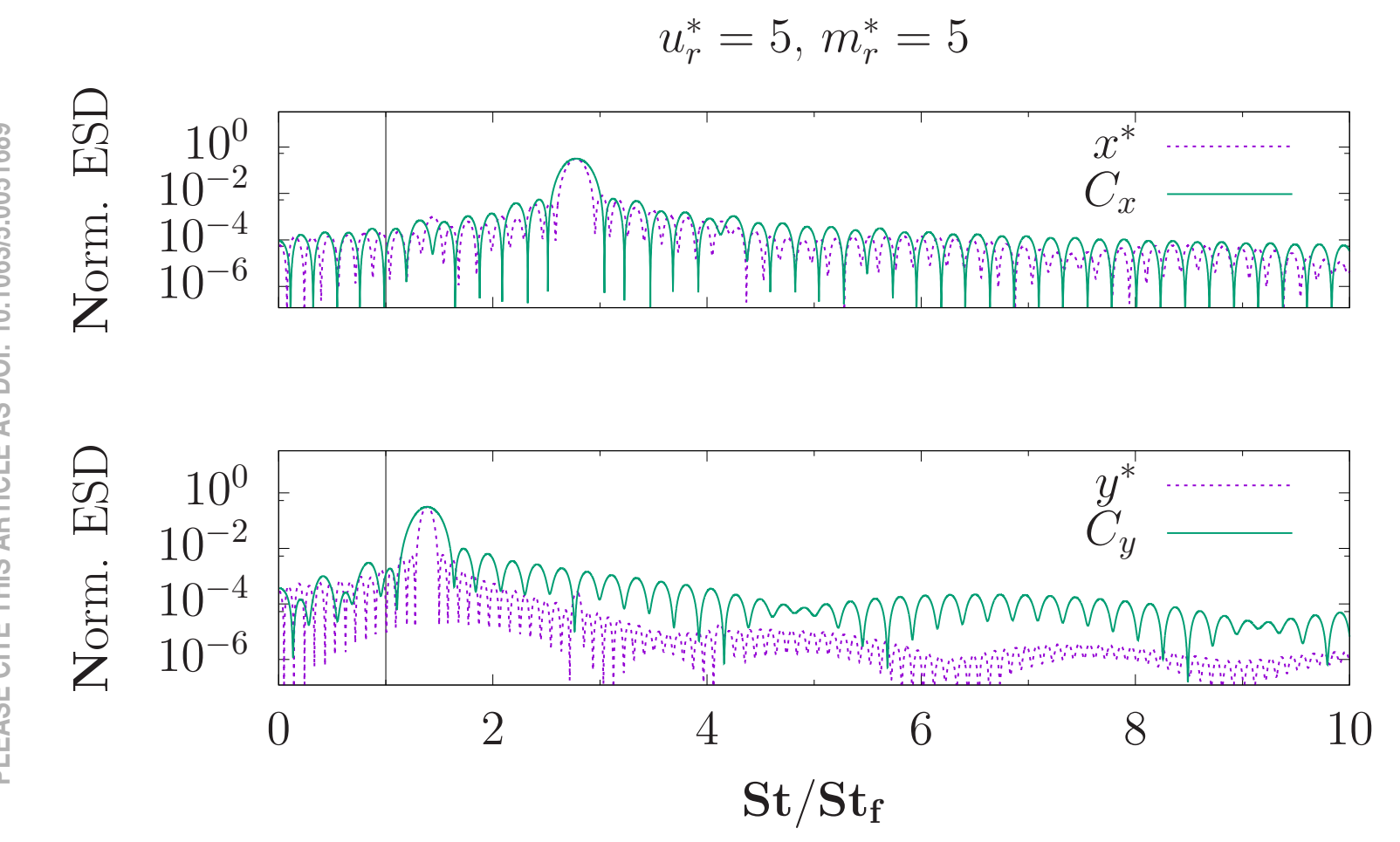




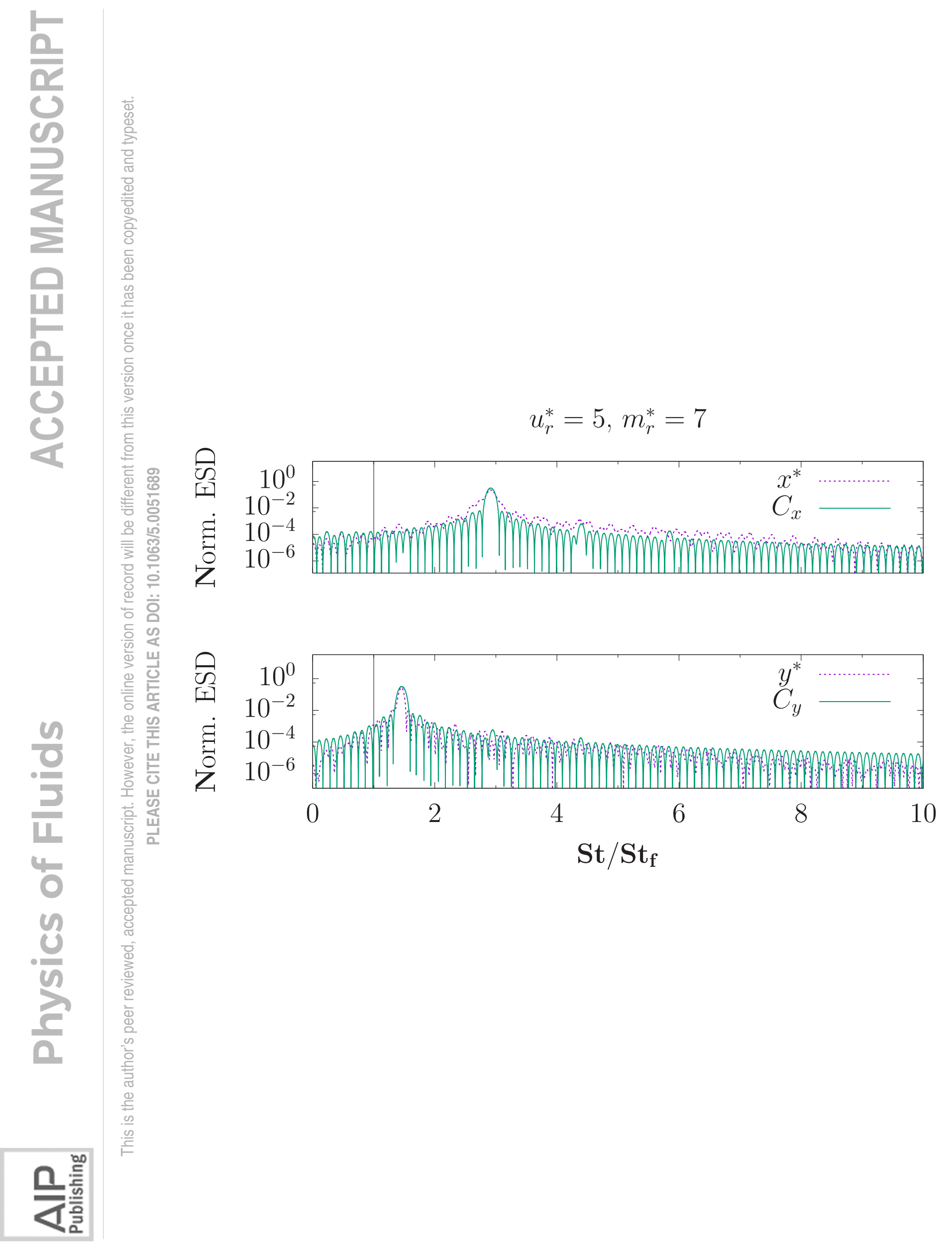




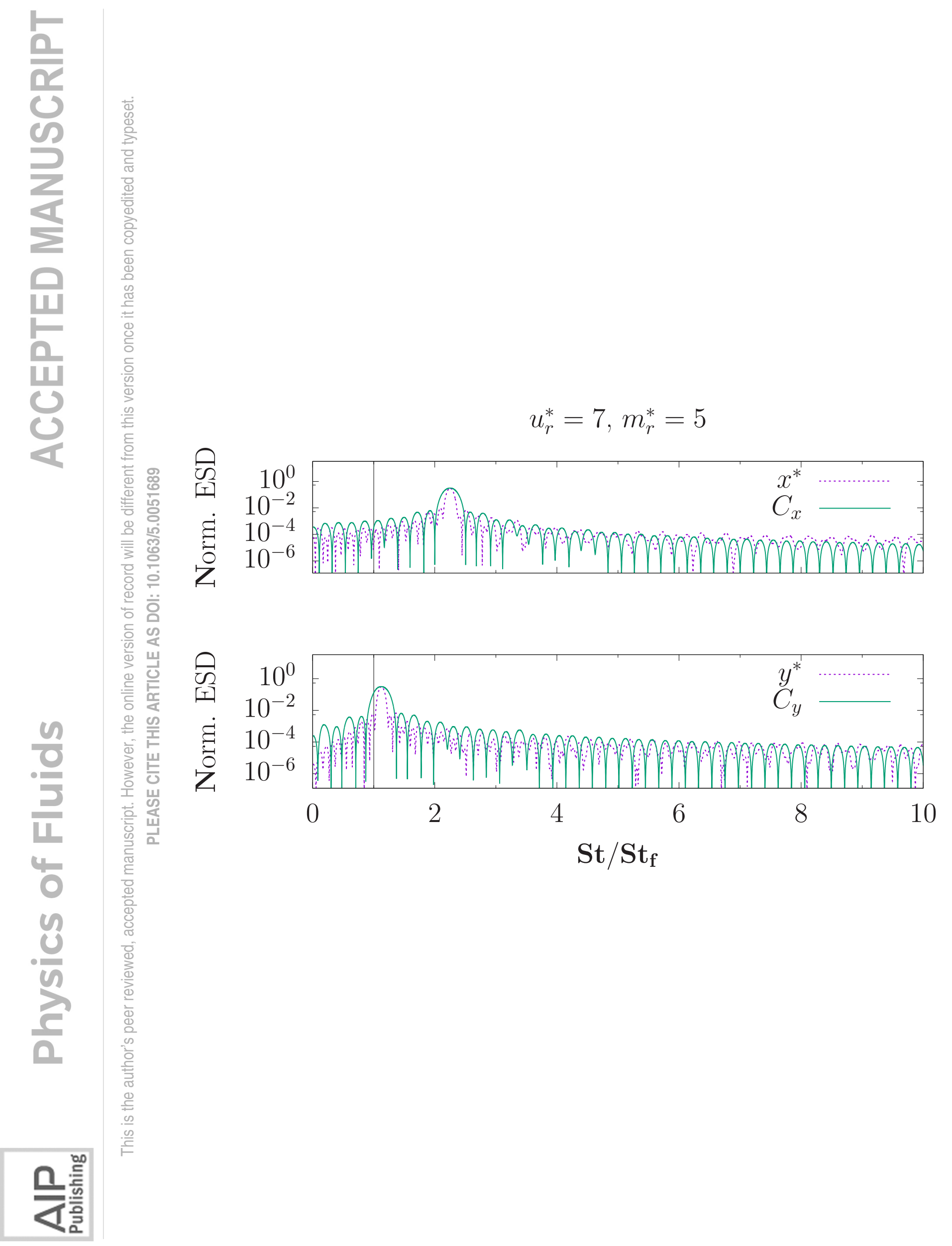




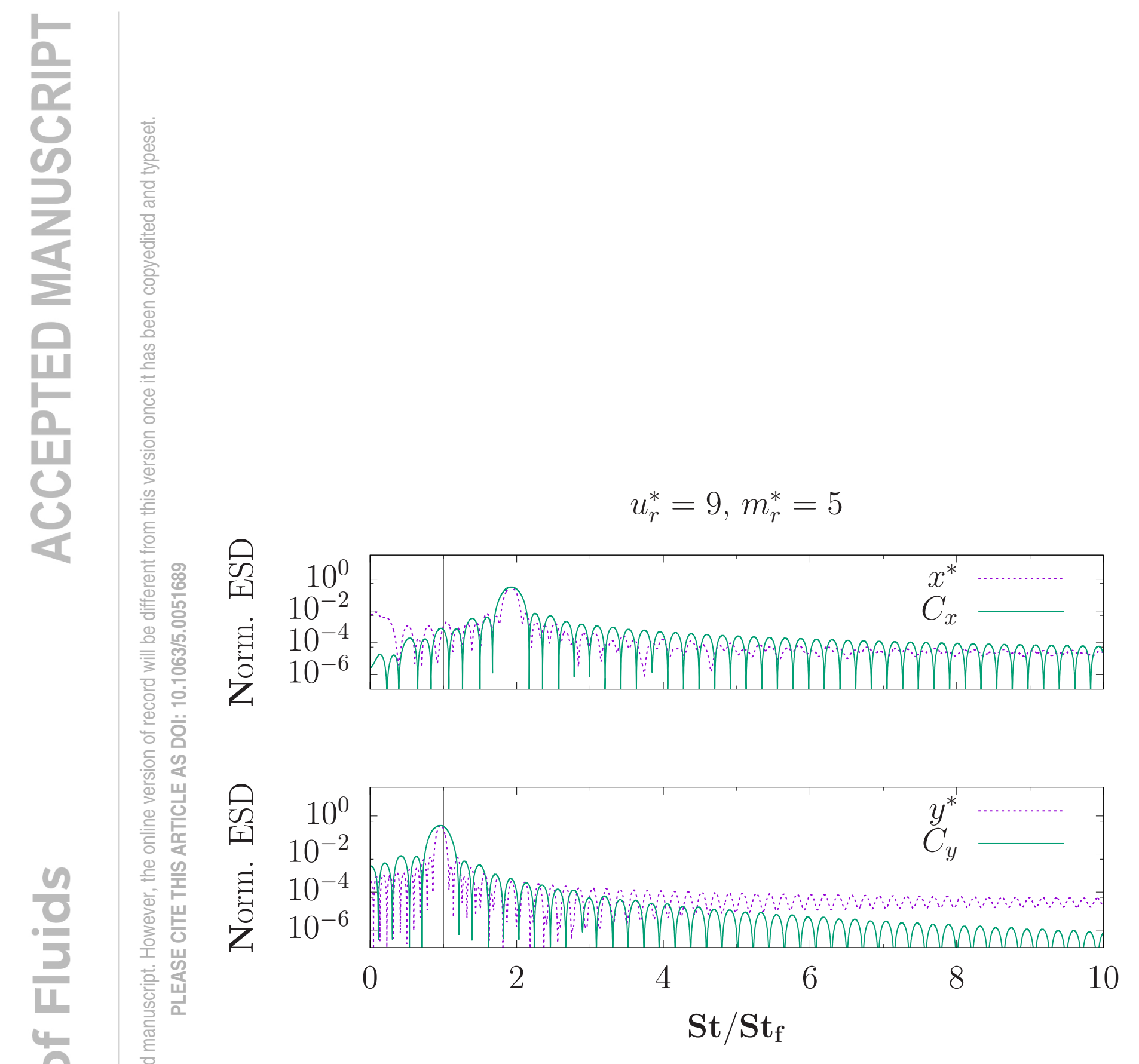



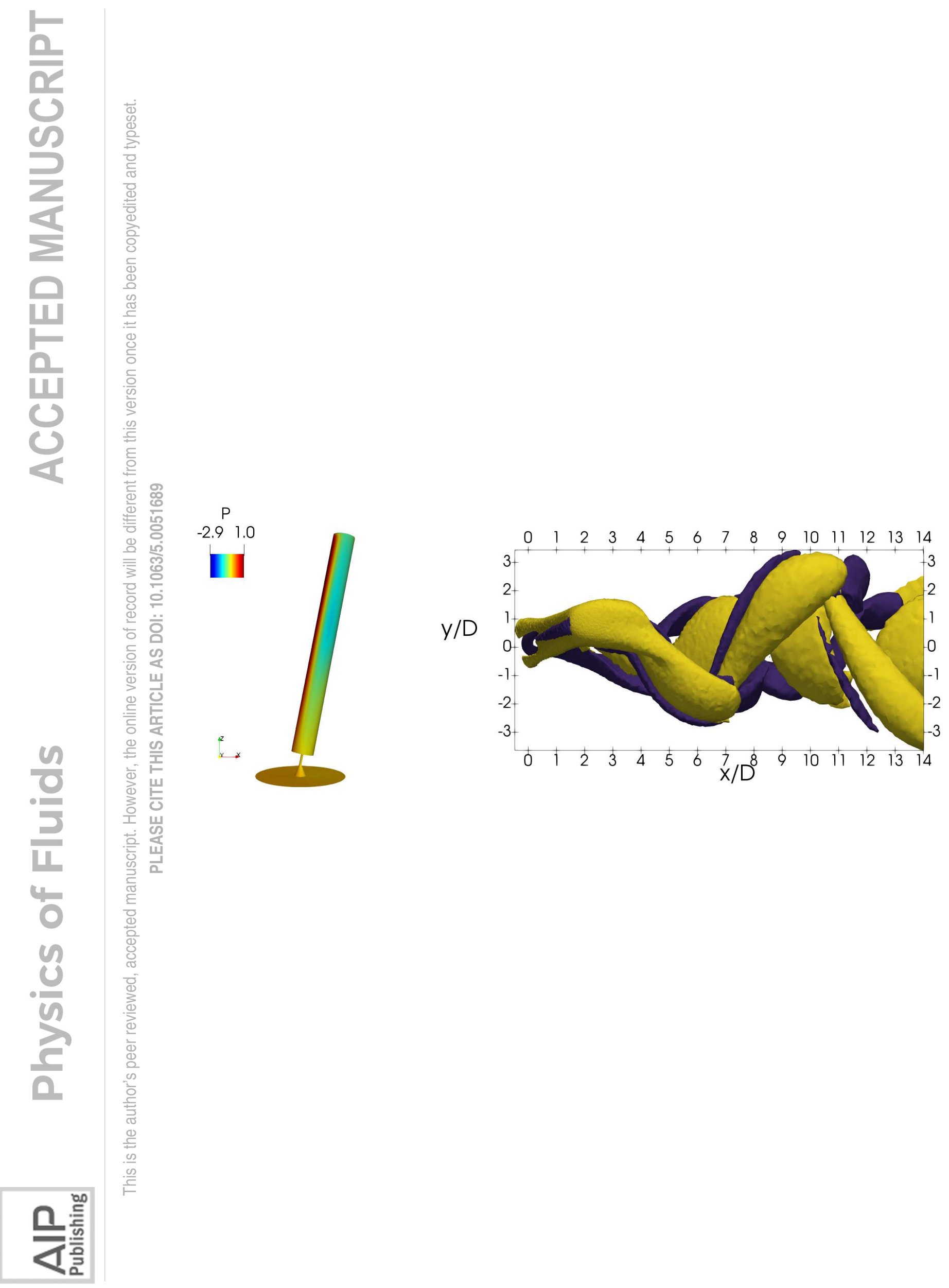


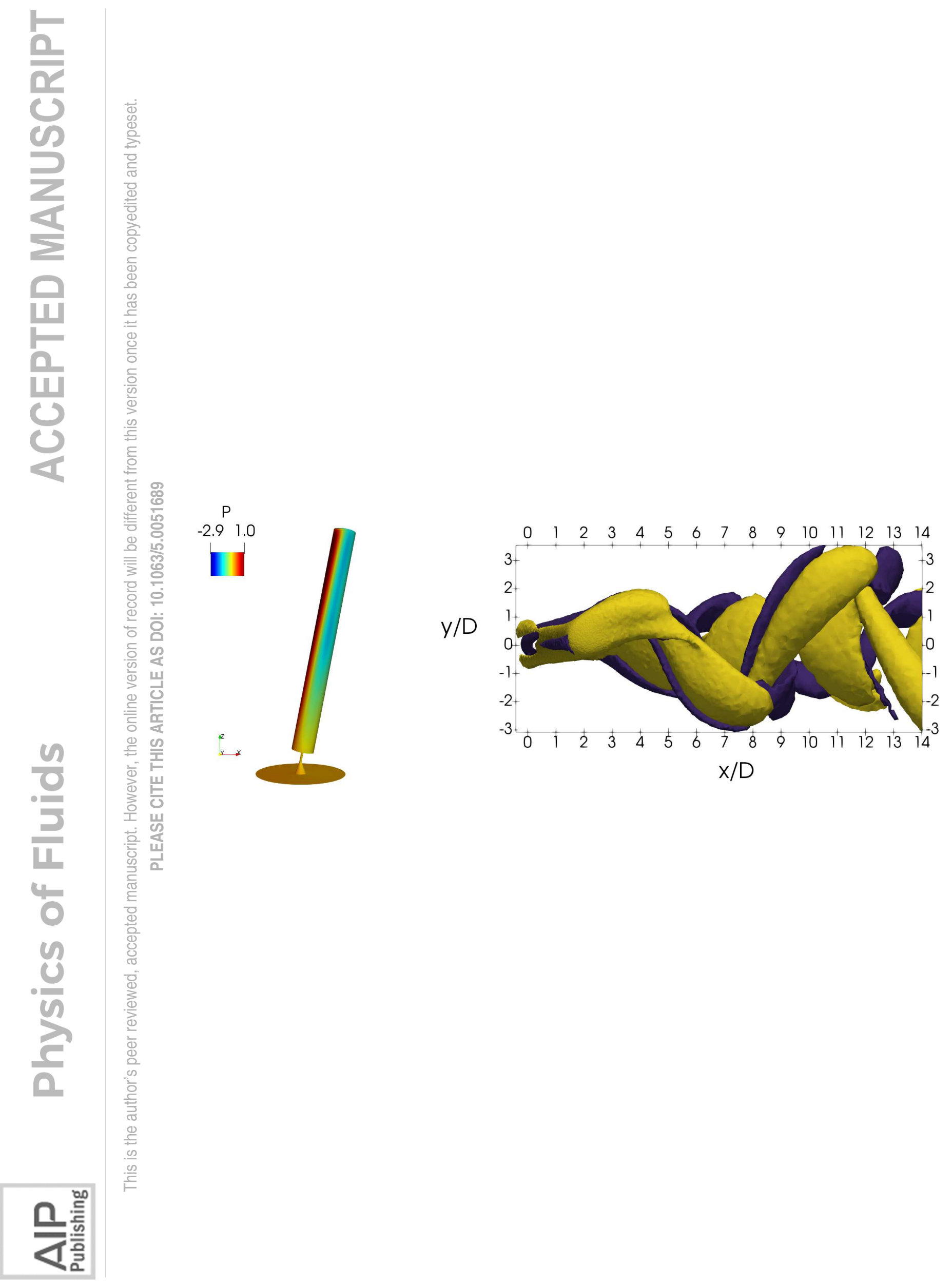




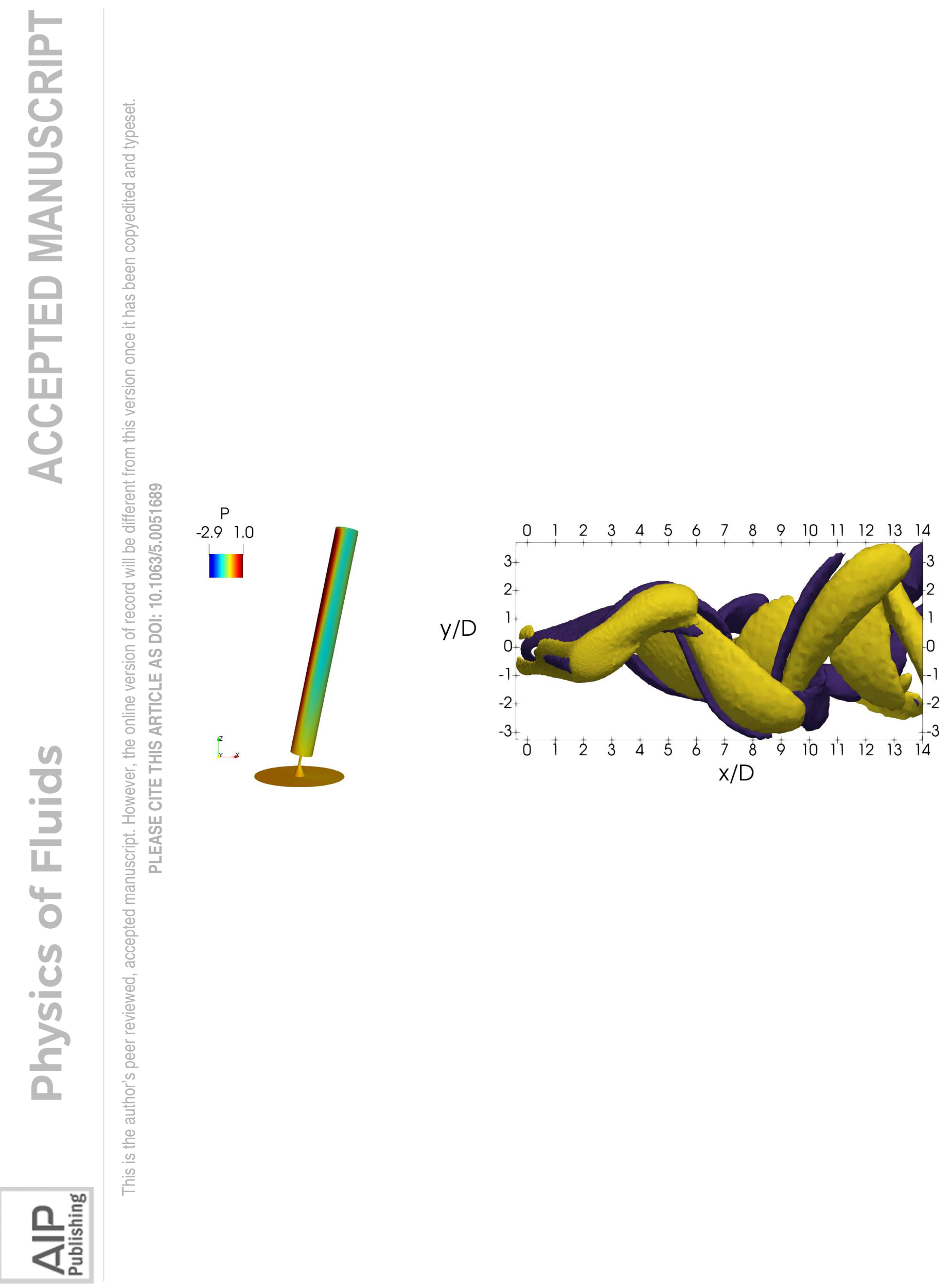



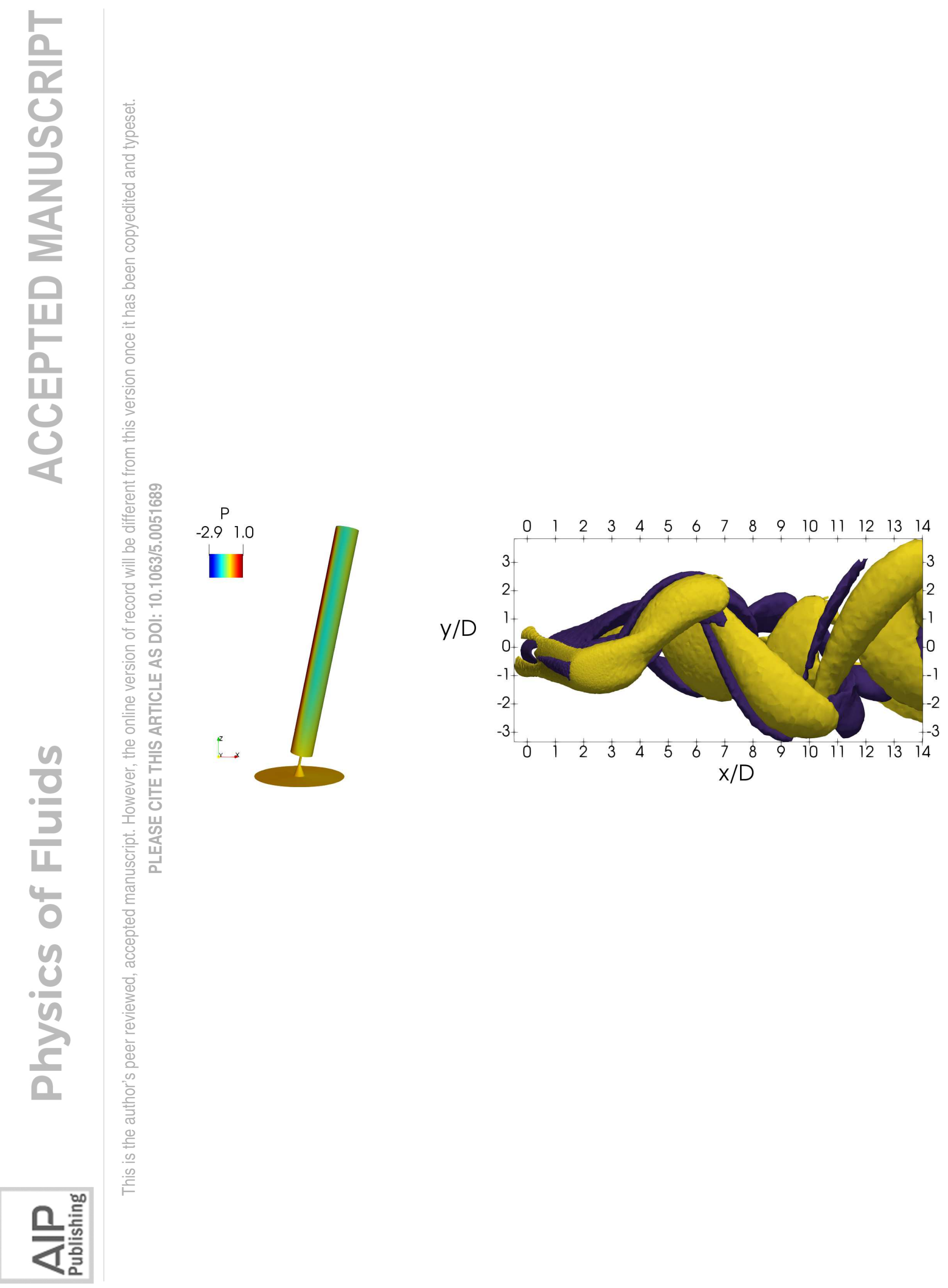

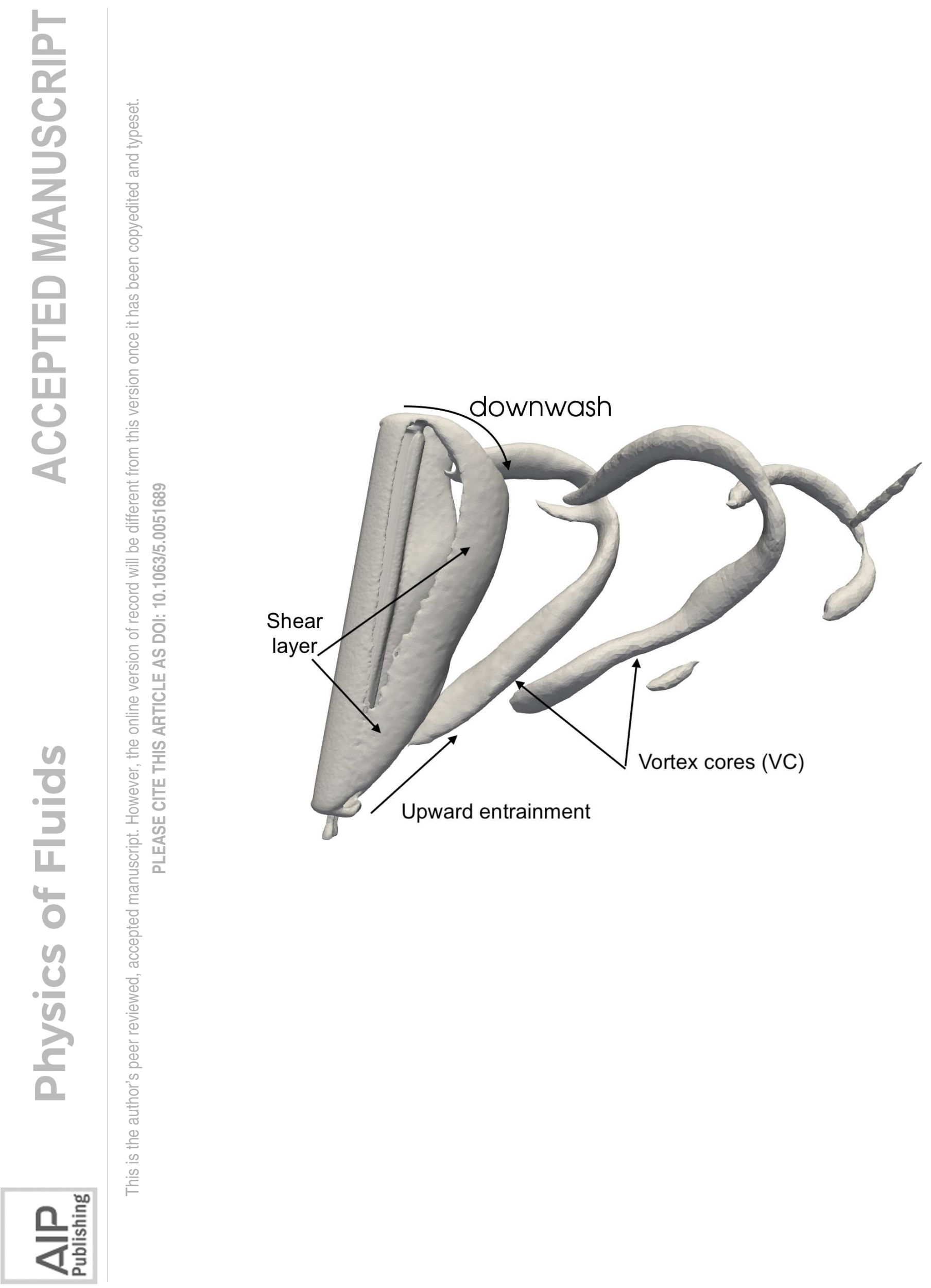


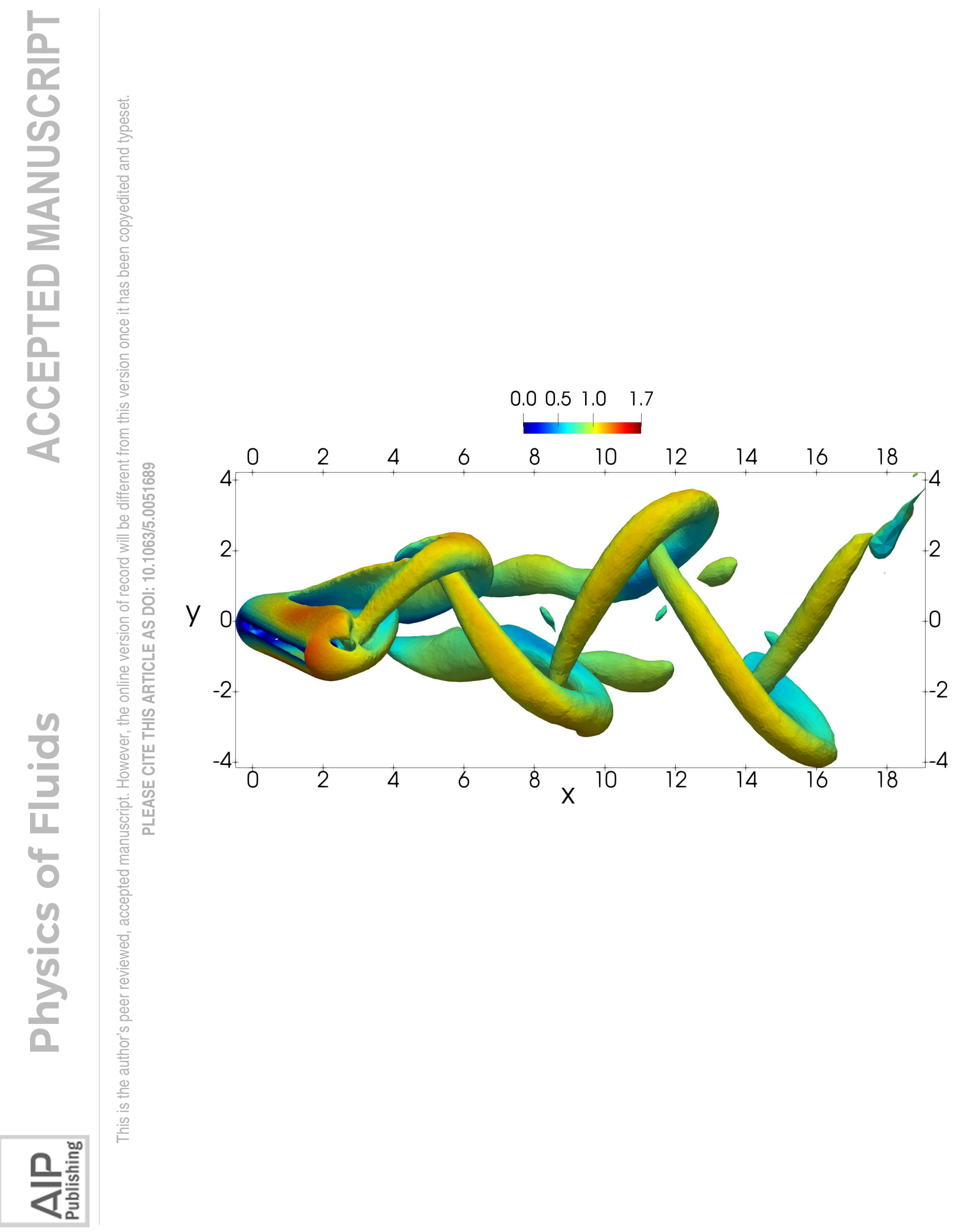



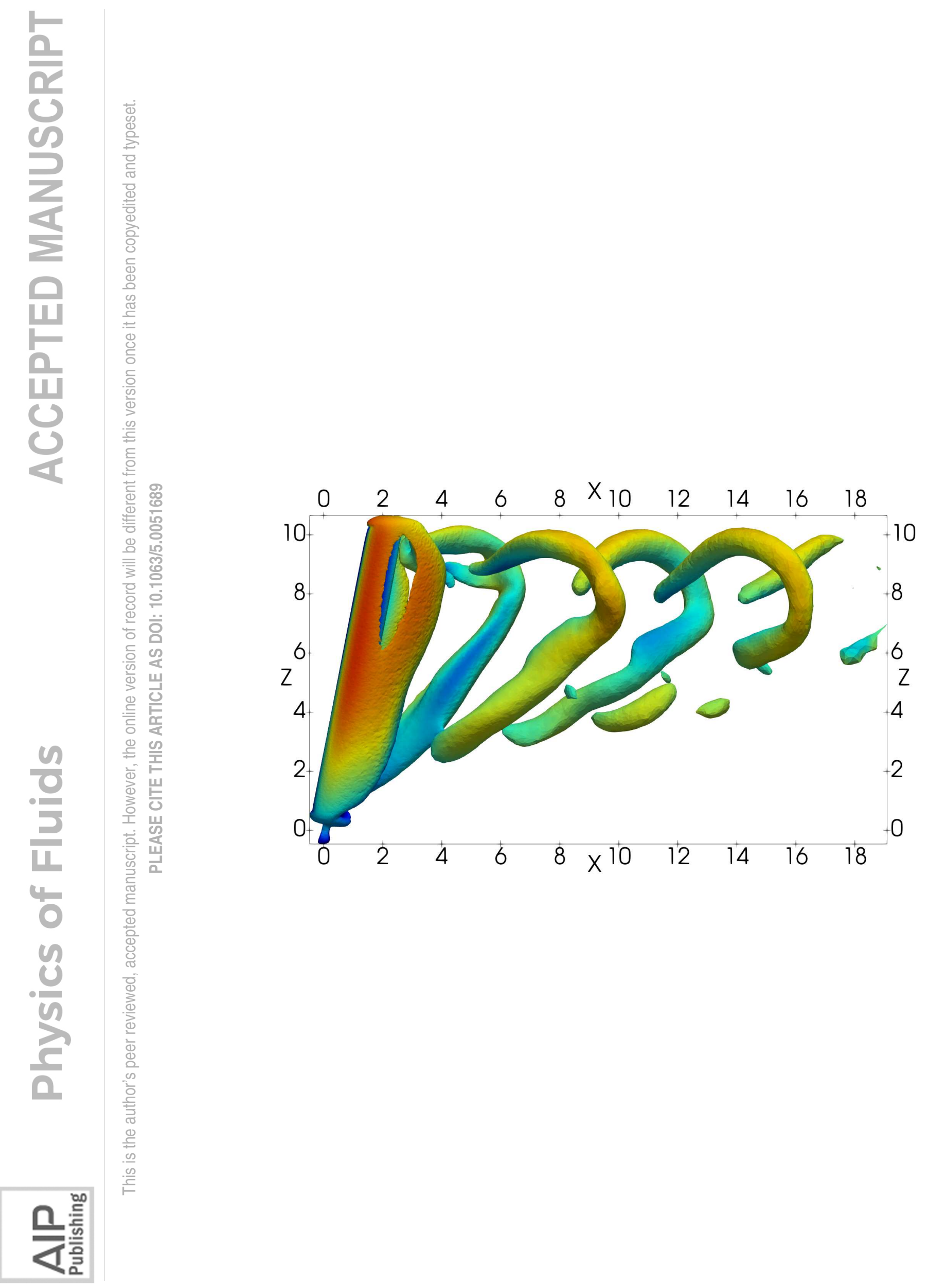


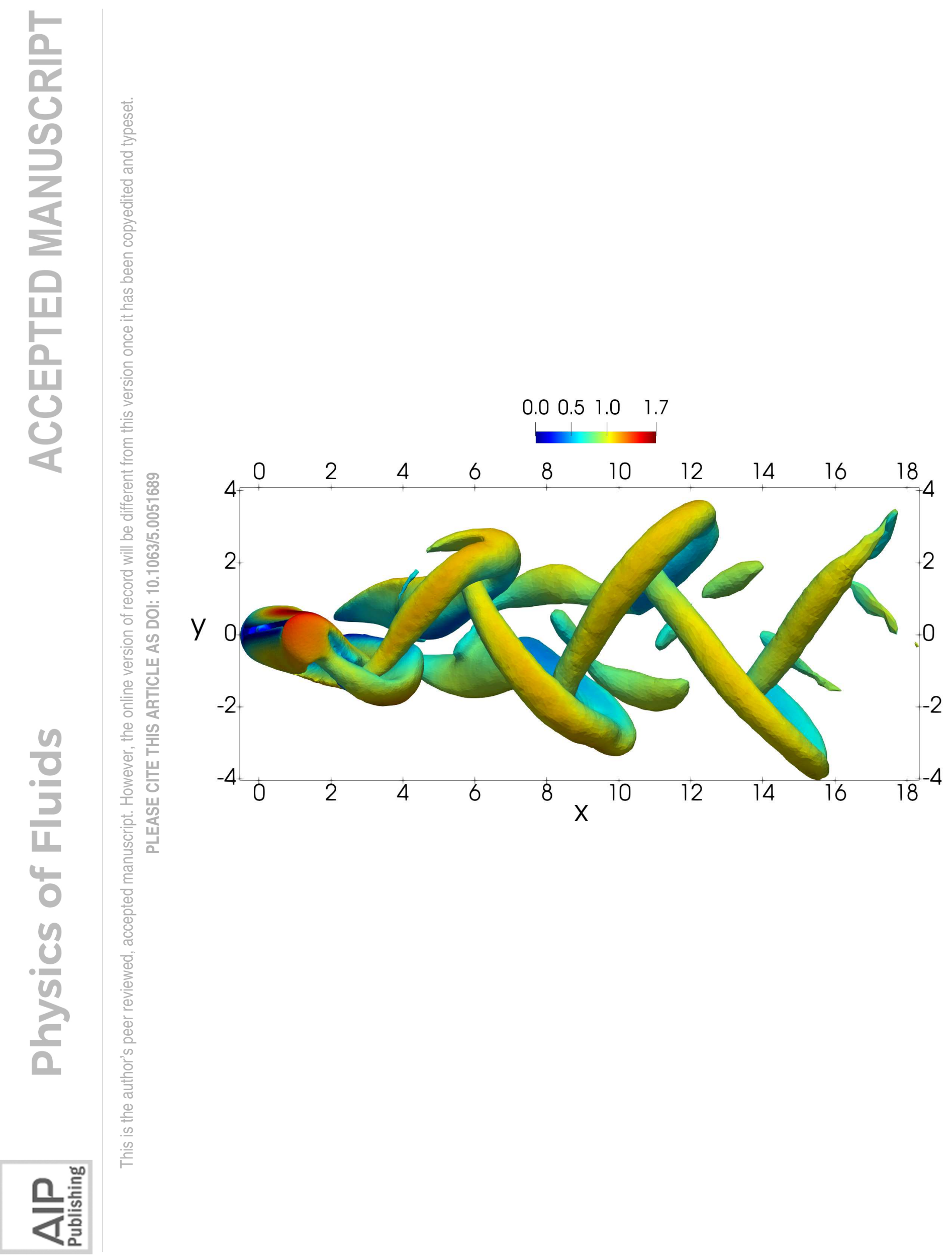



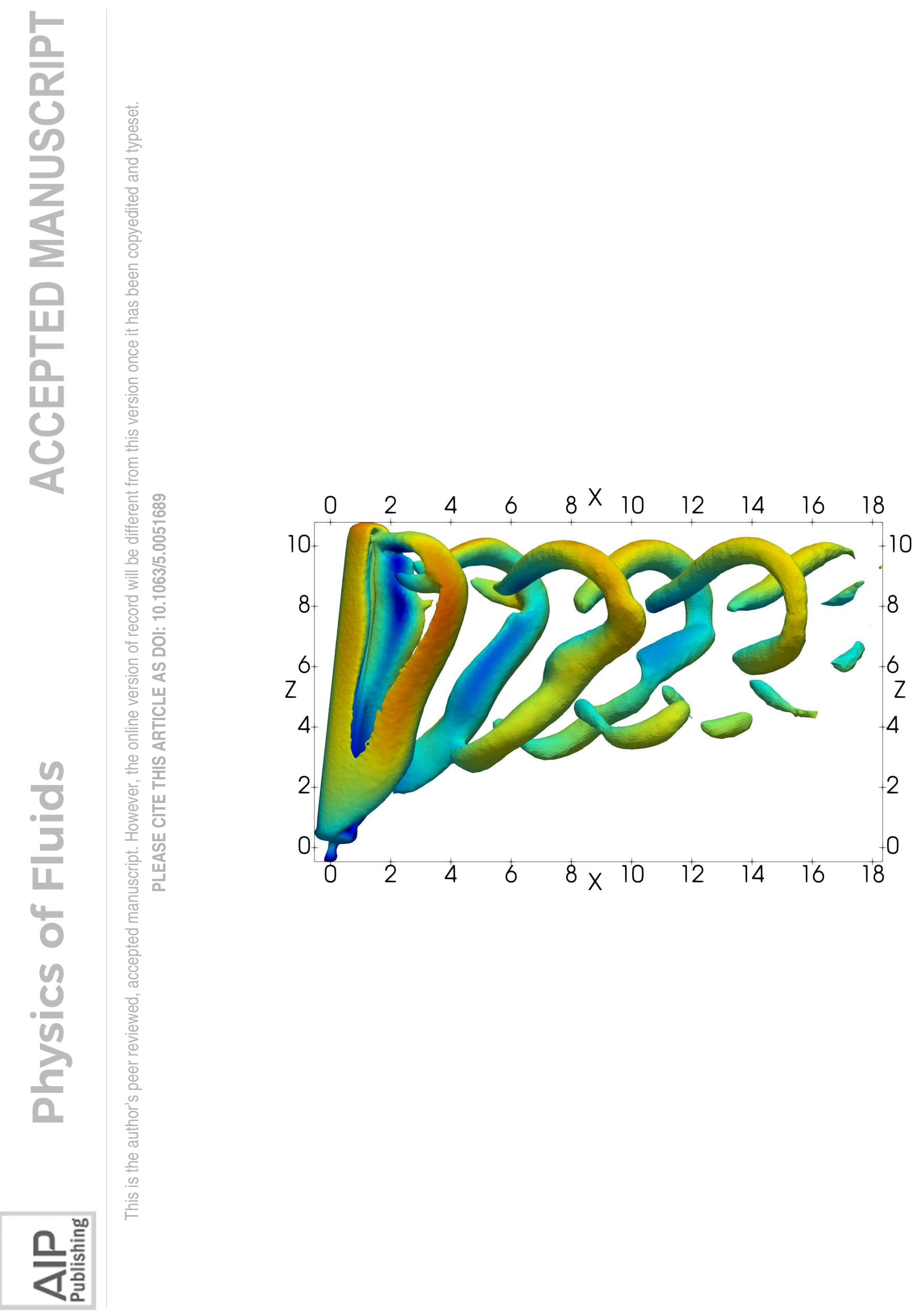


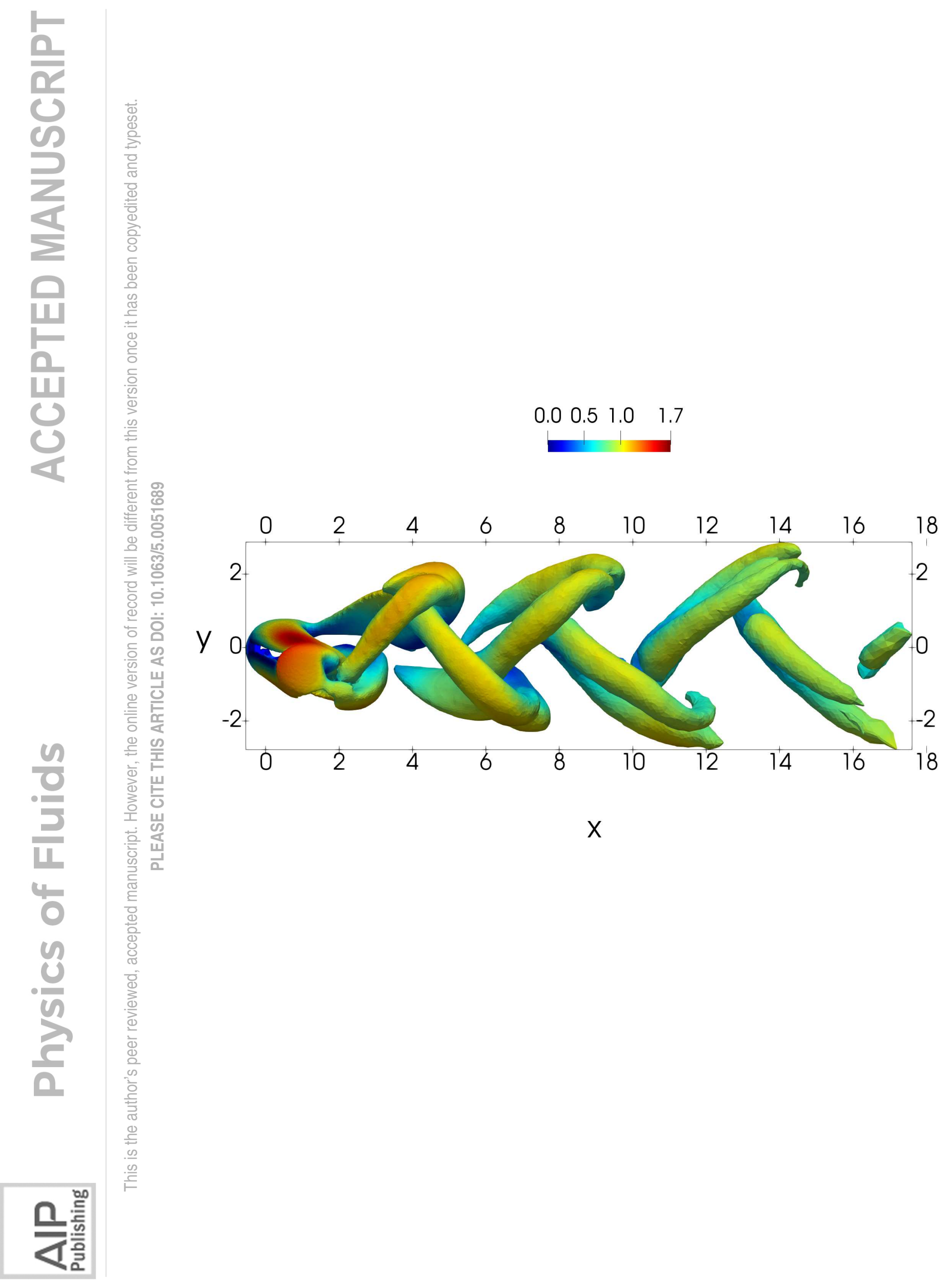



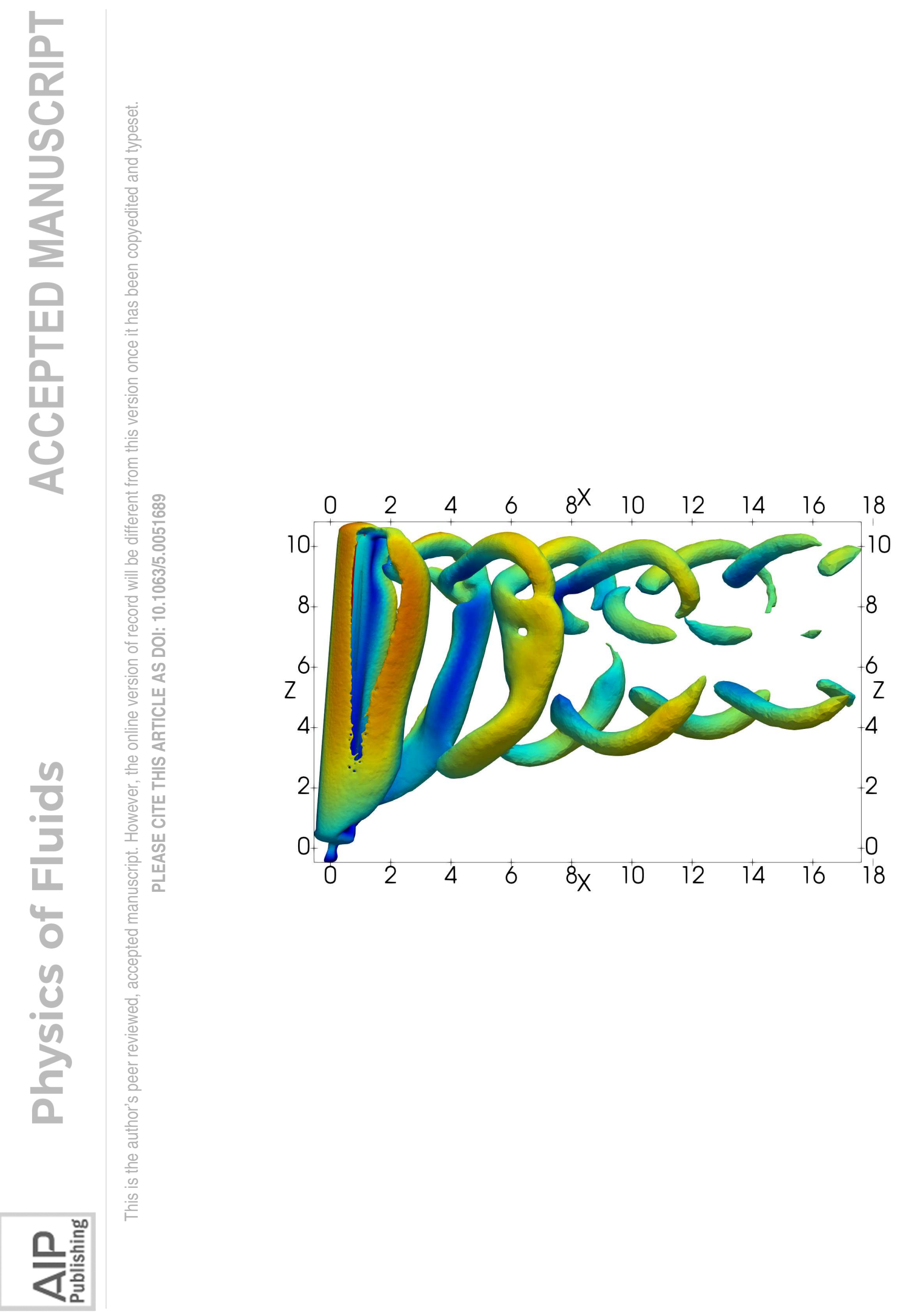


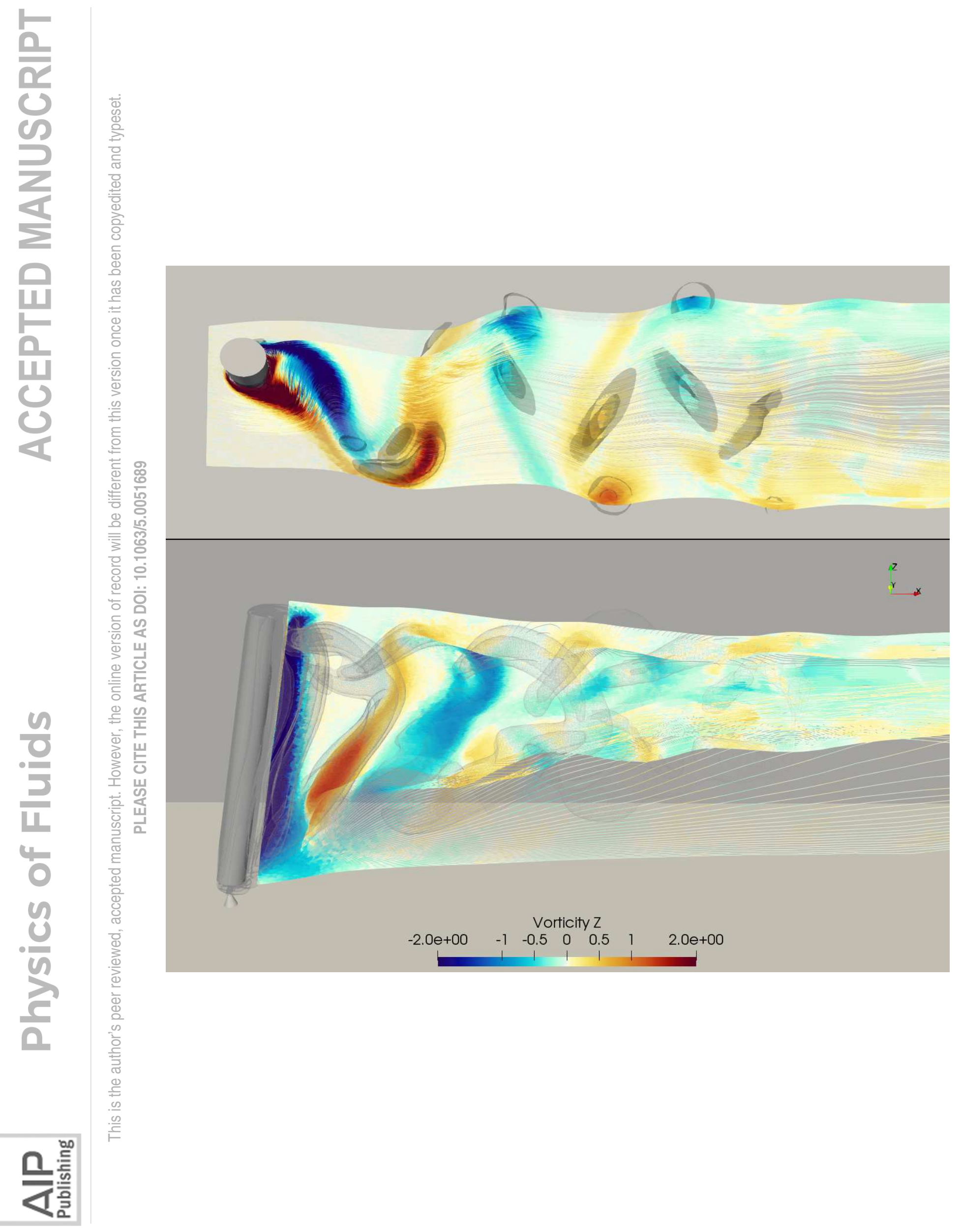



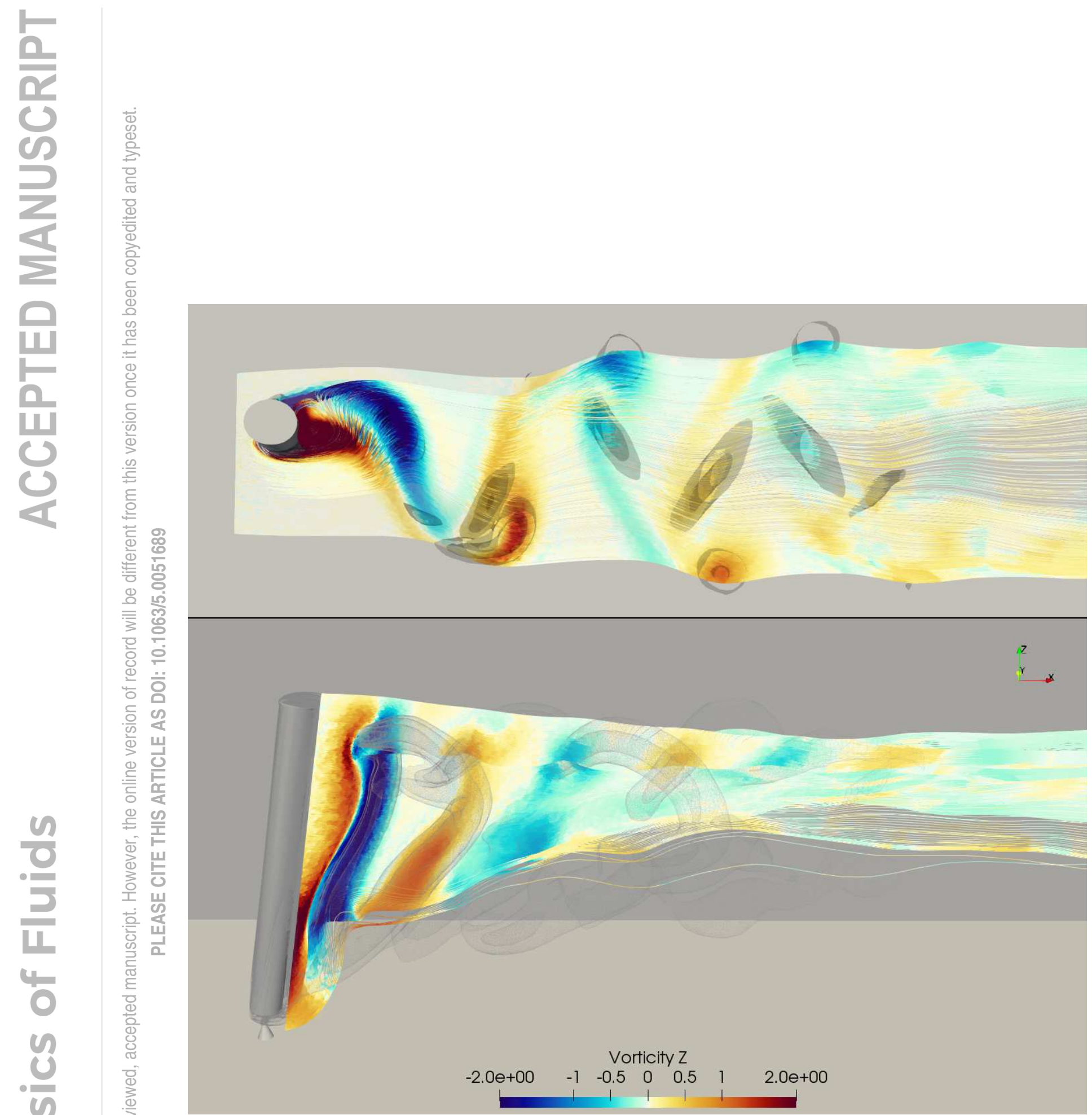

는 


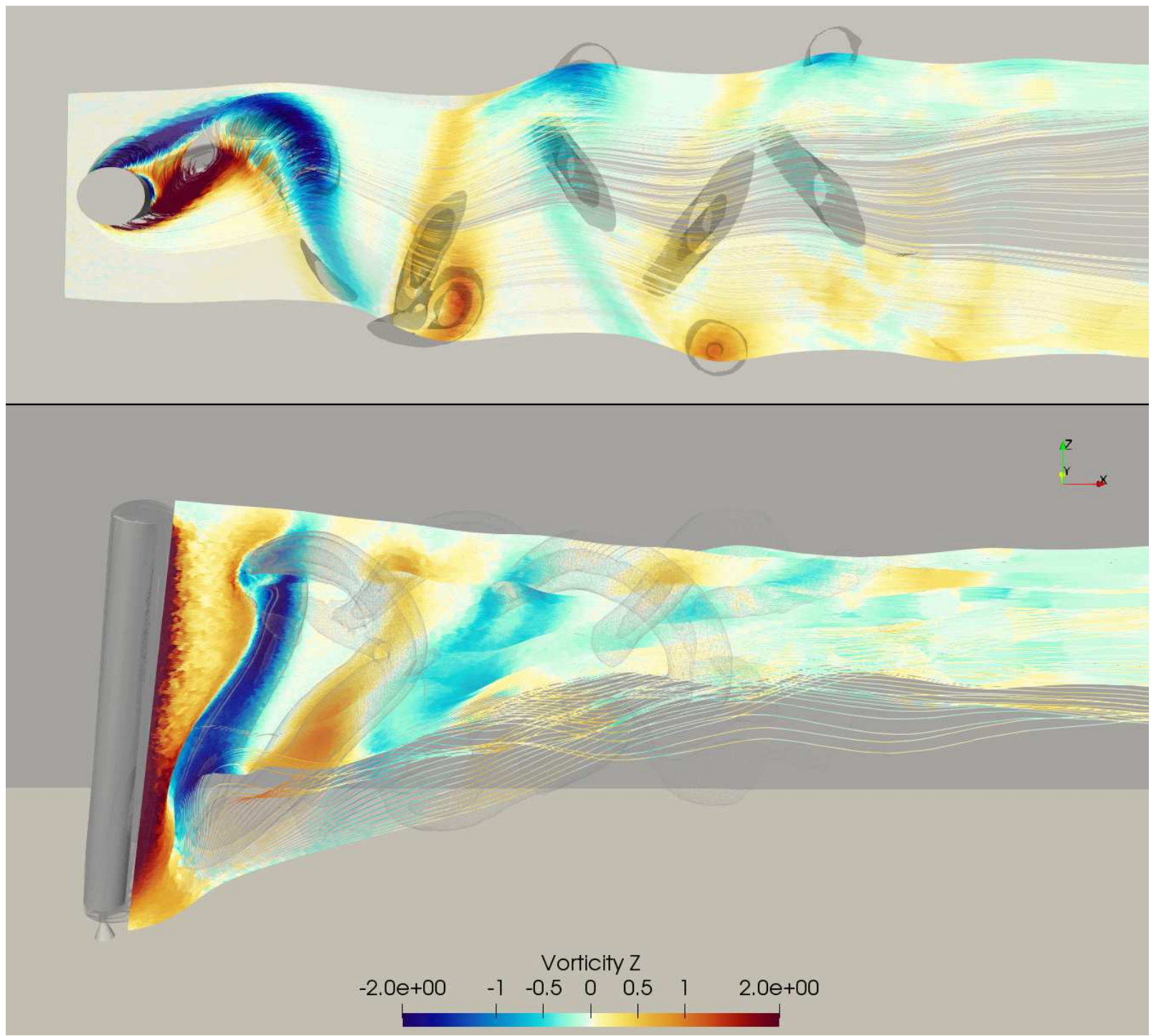

兄耪言 


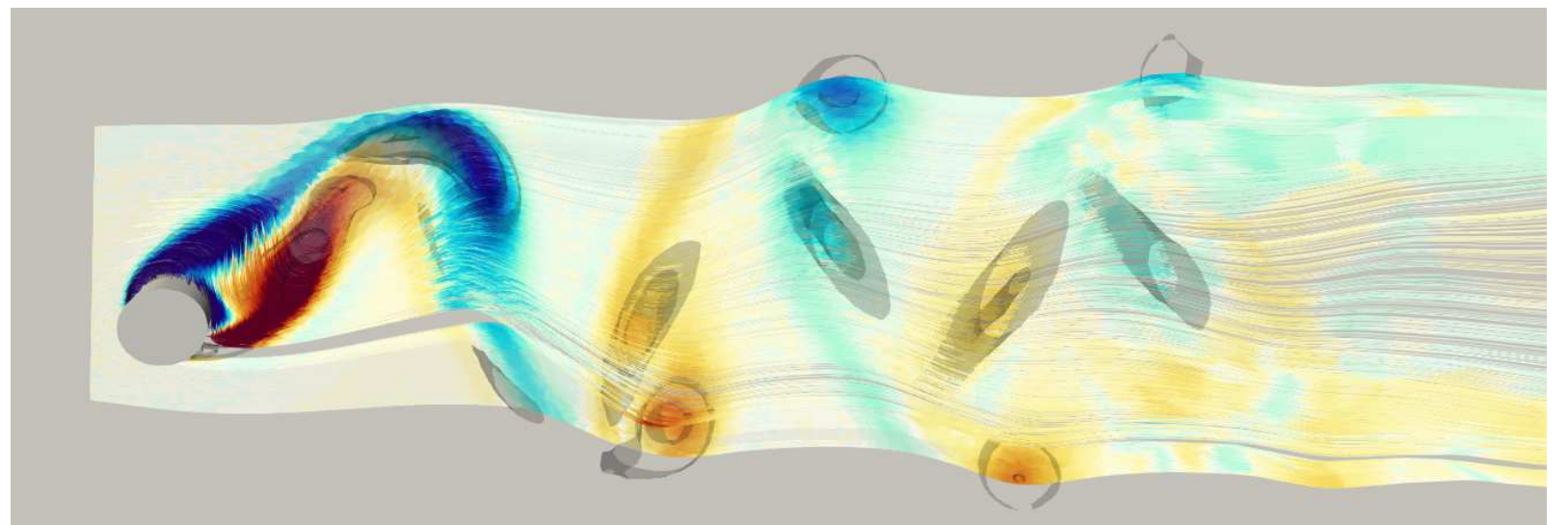

0
0
5
11
4
0
0
0
0

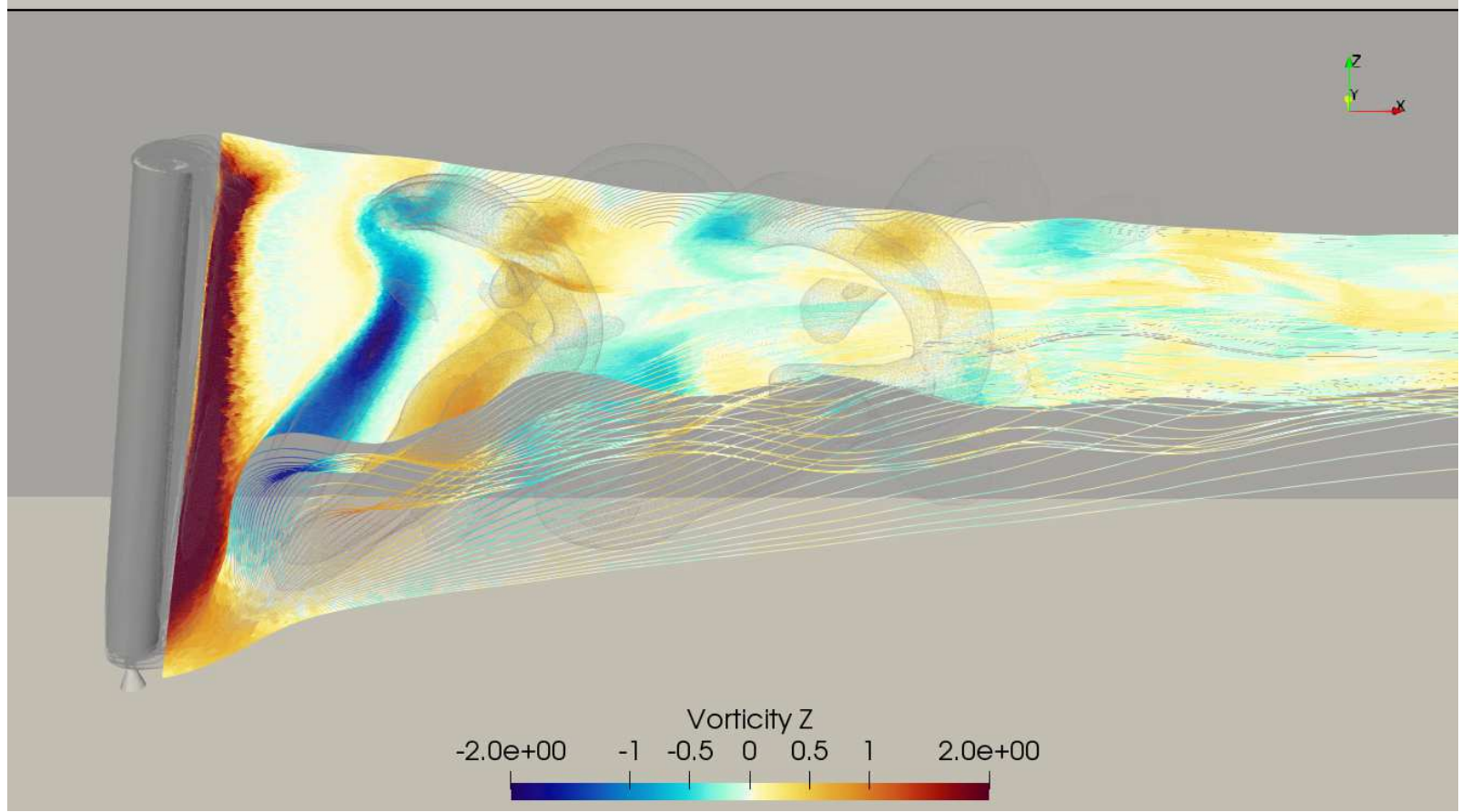

兄喜譬 

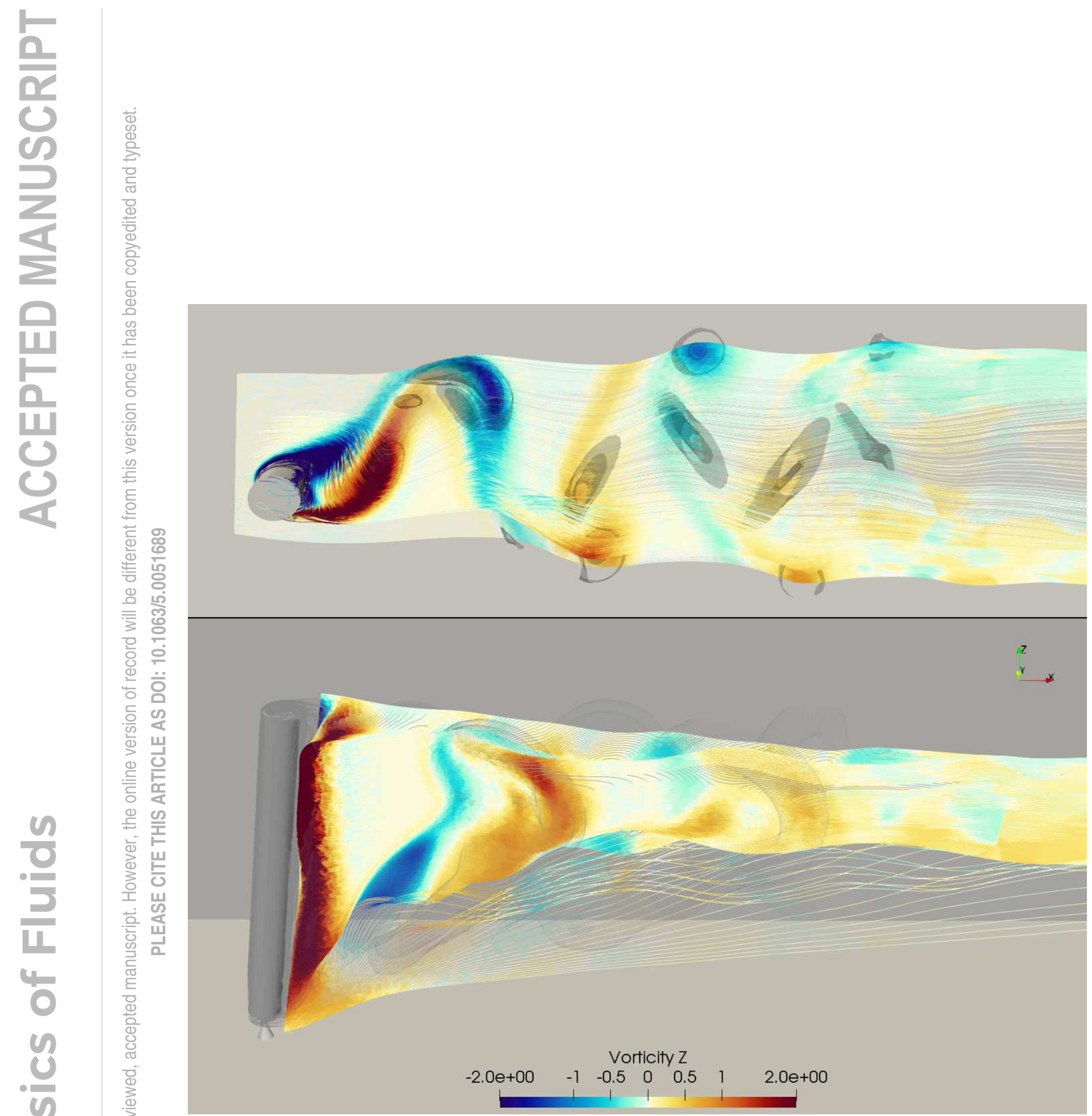

는 

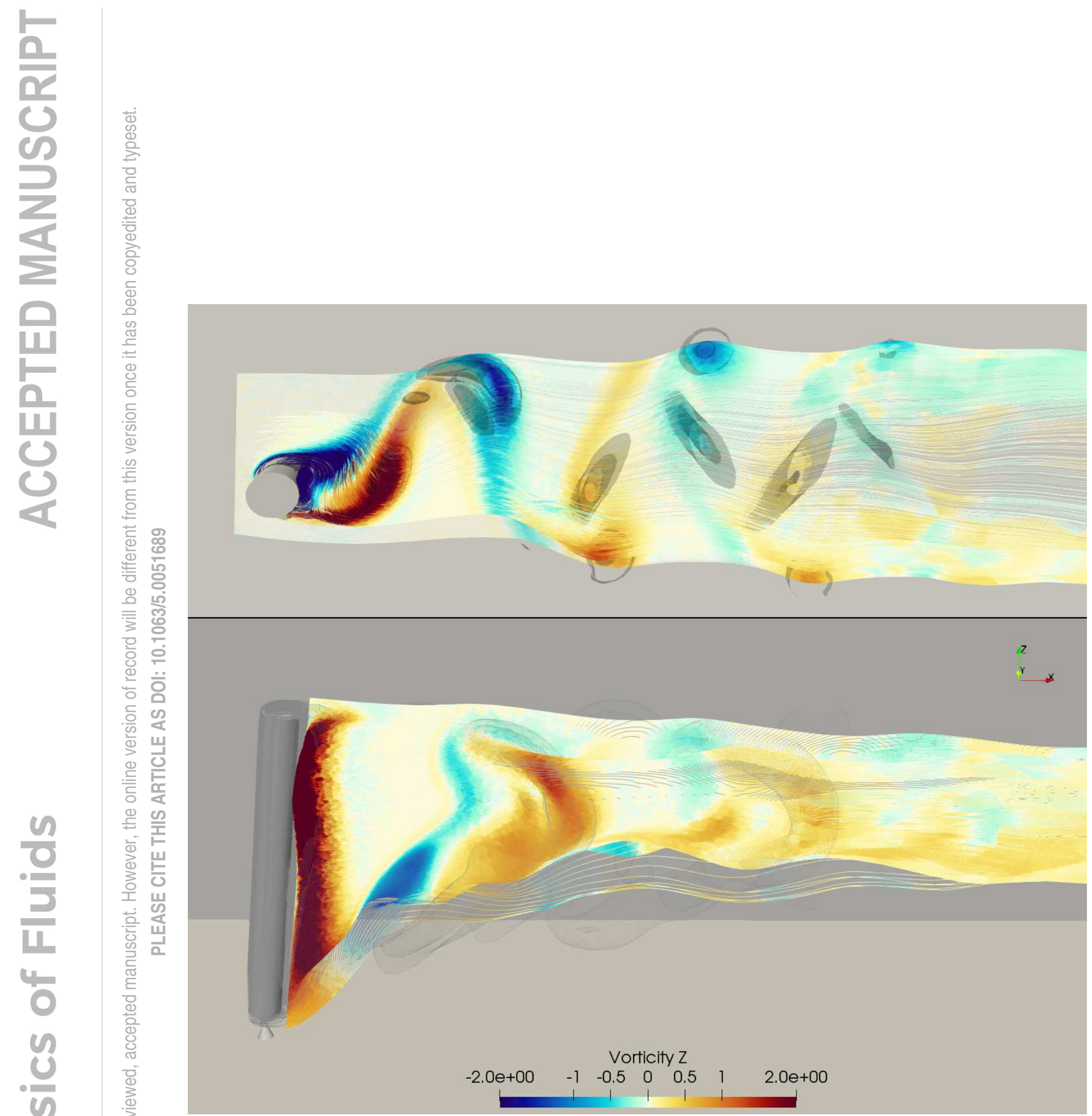

는 


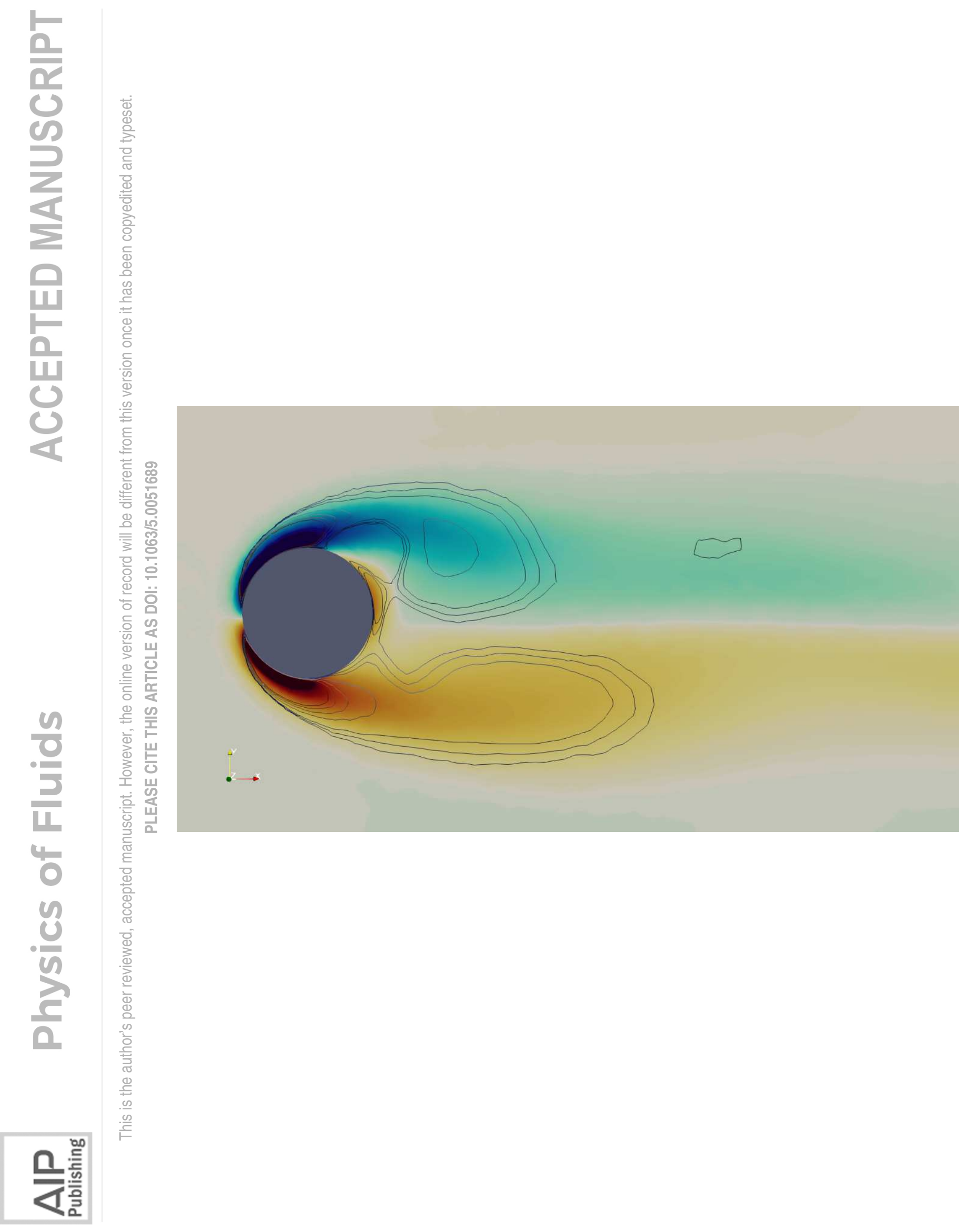




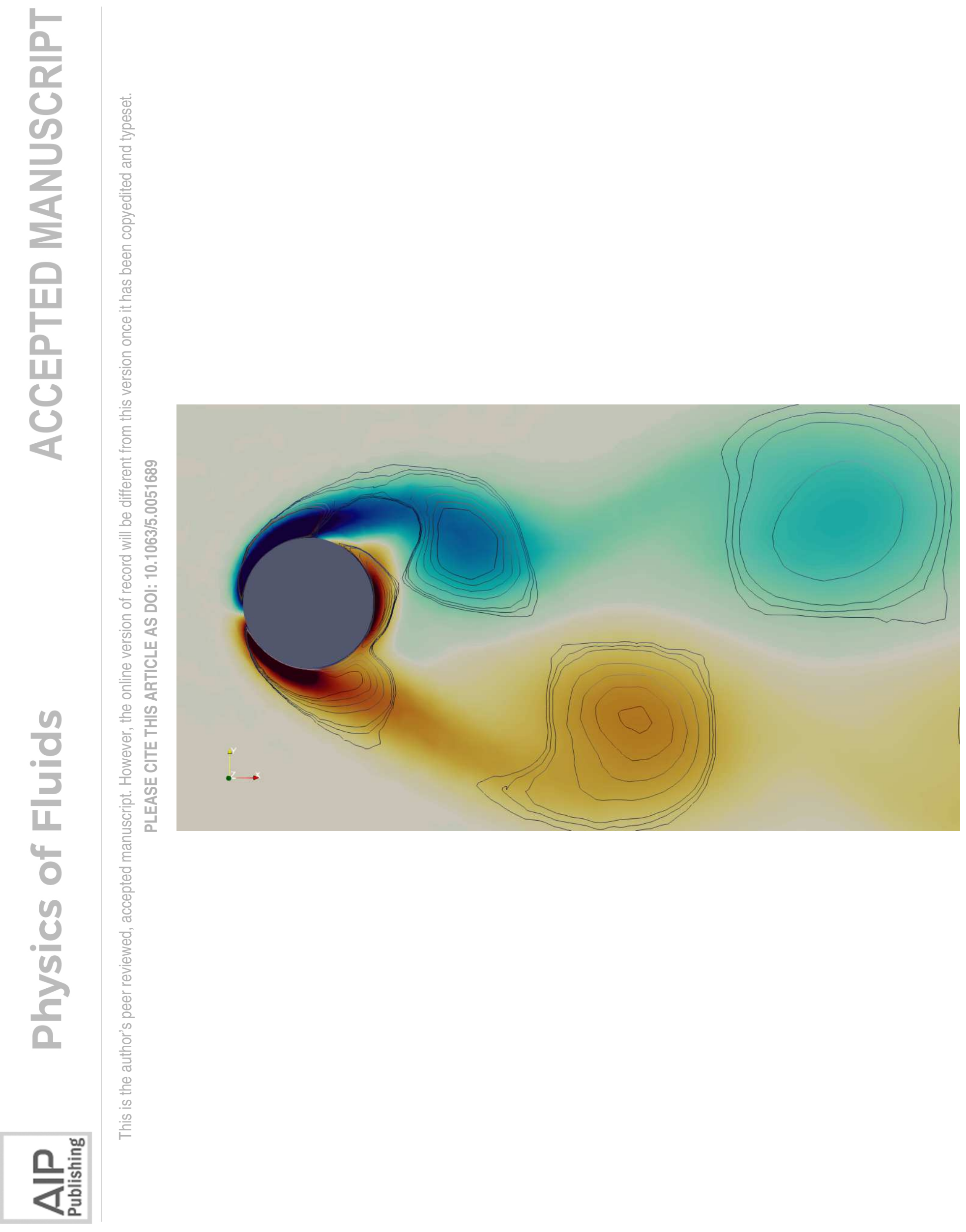




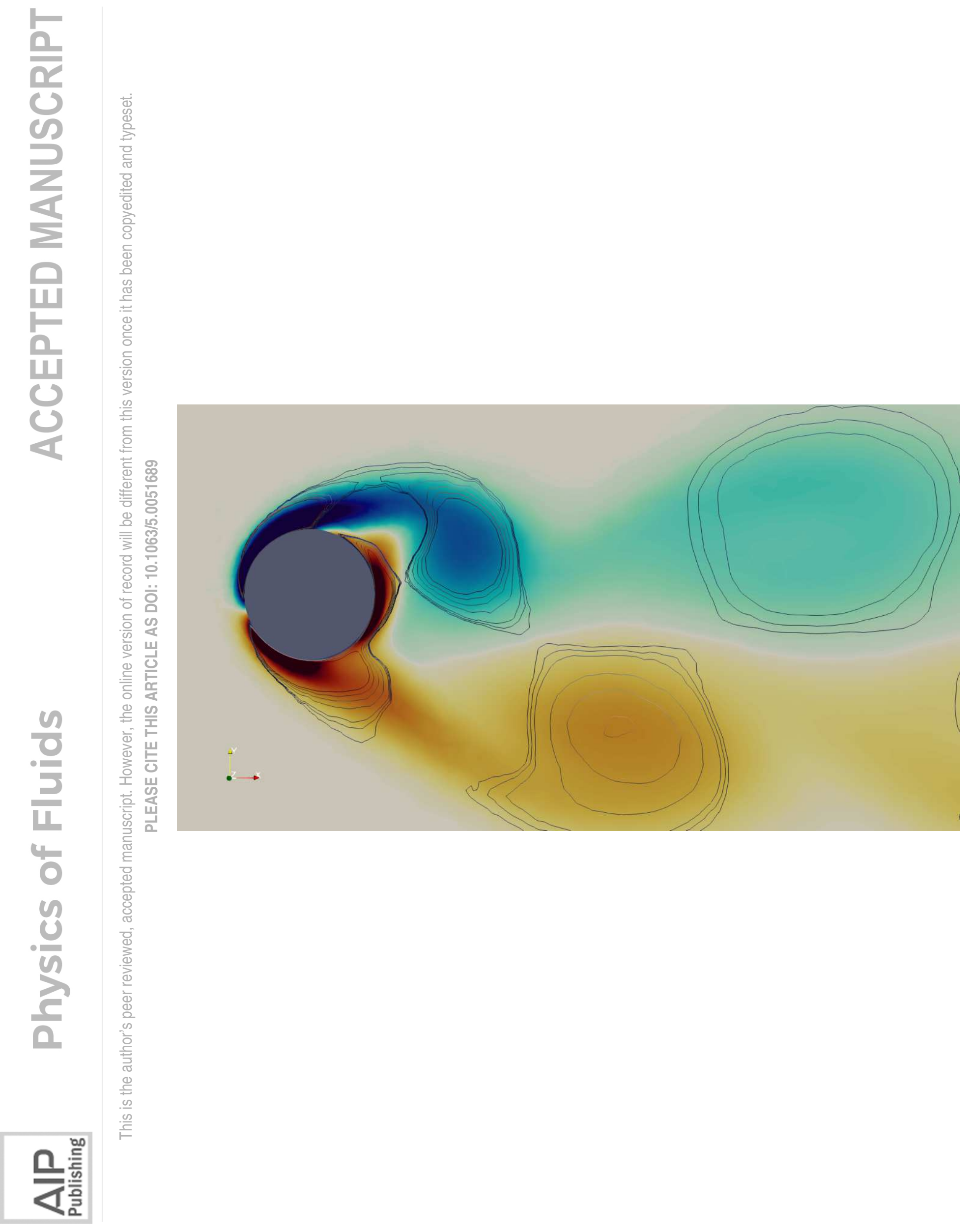




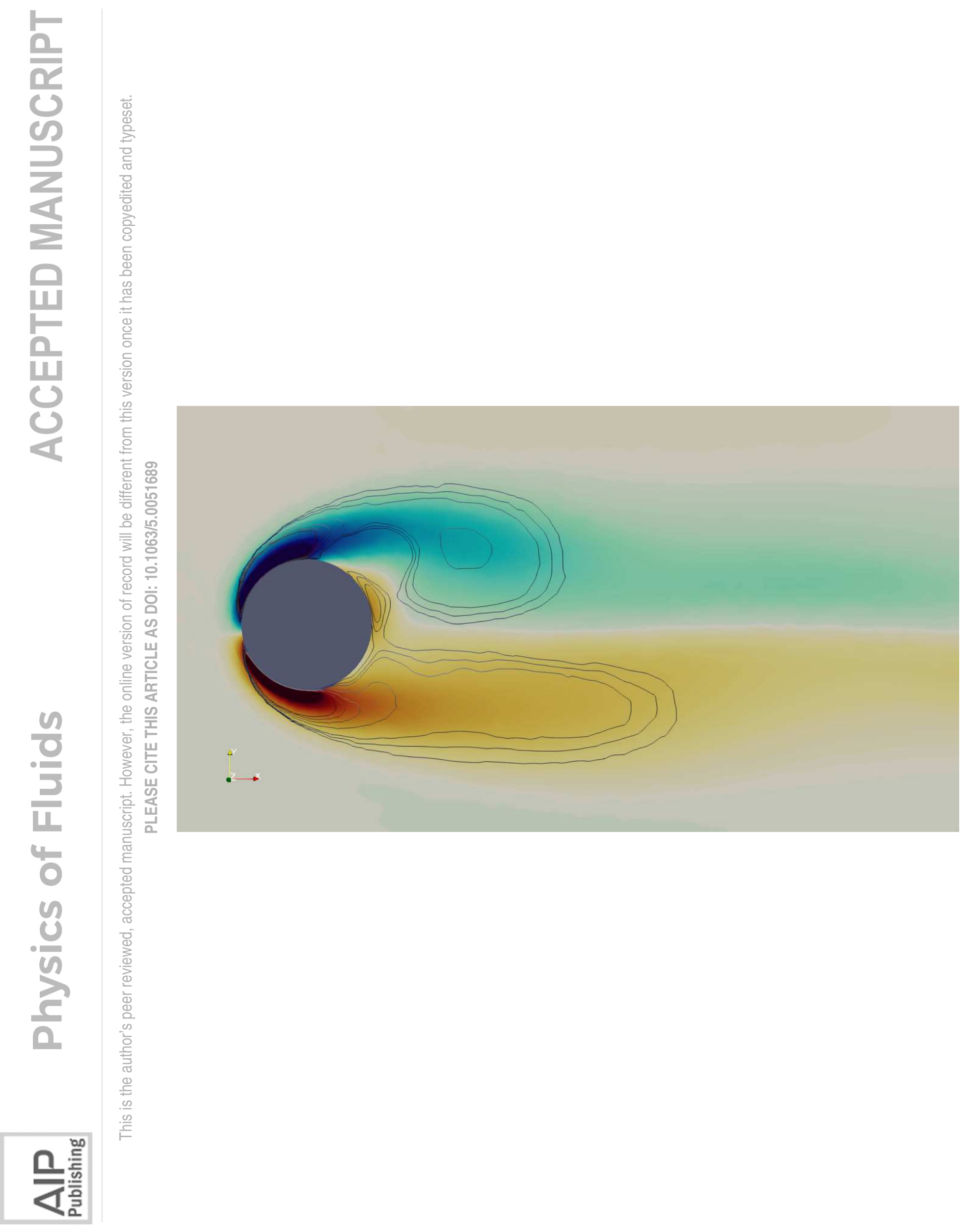




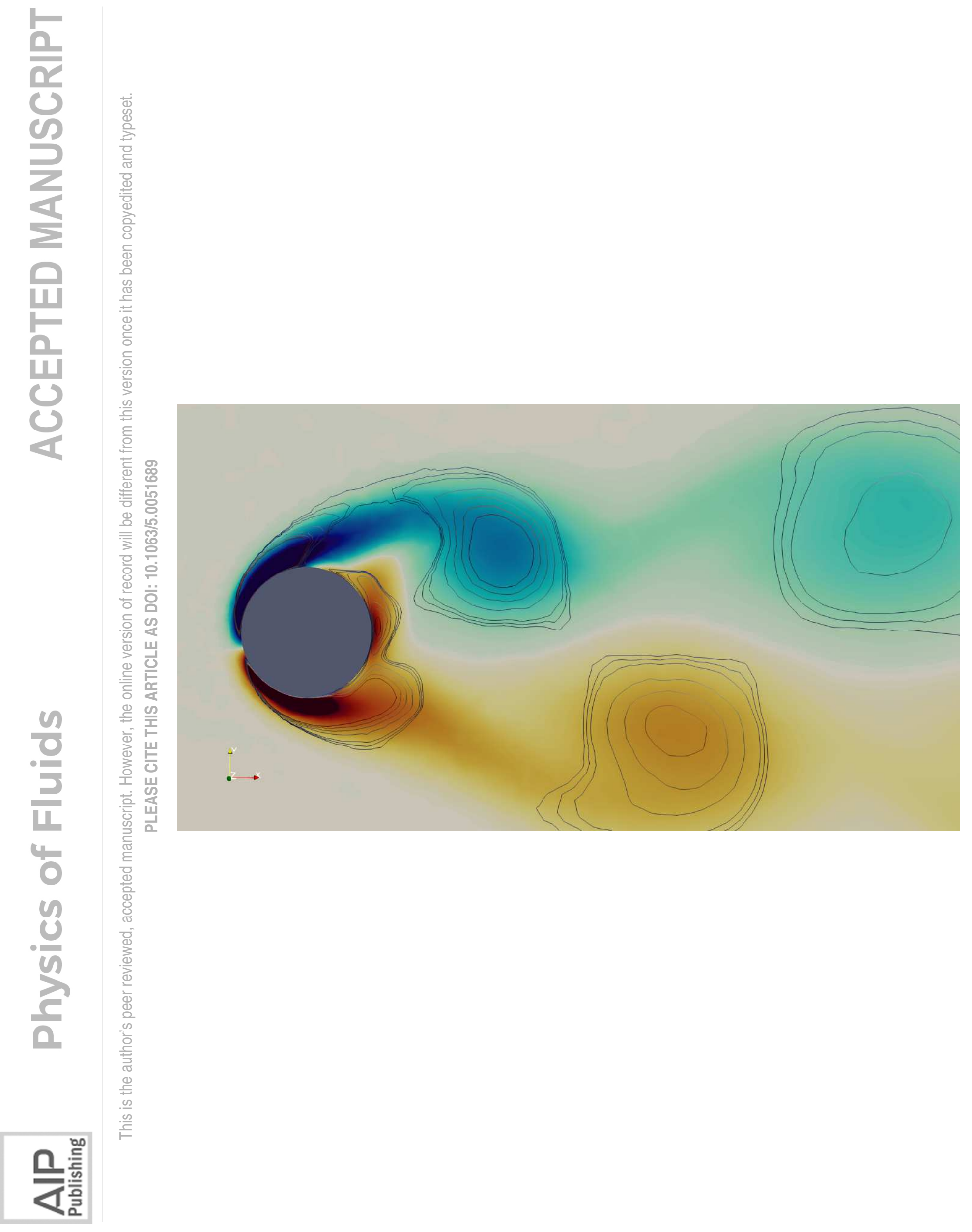




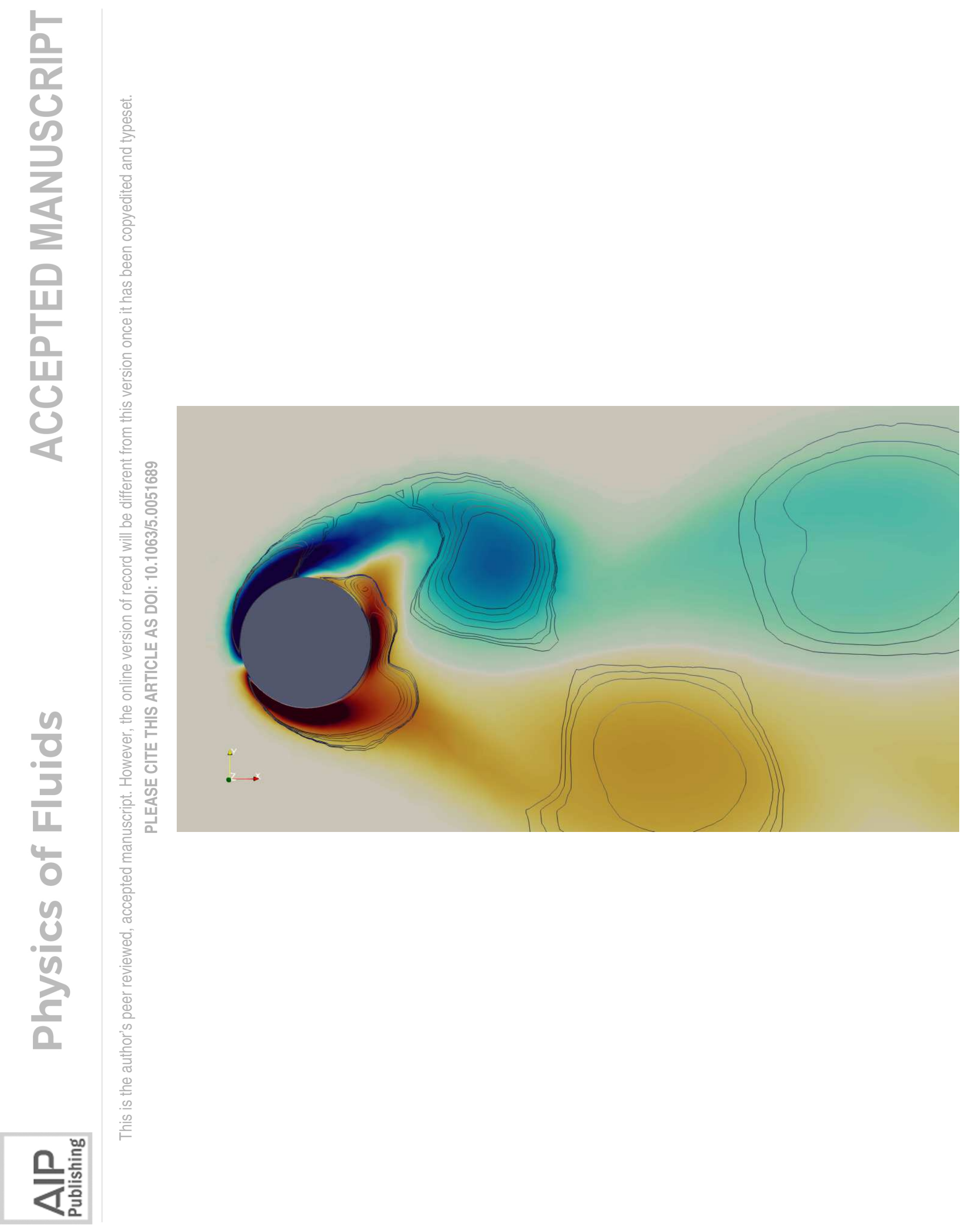




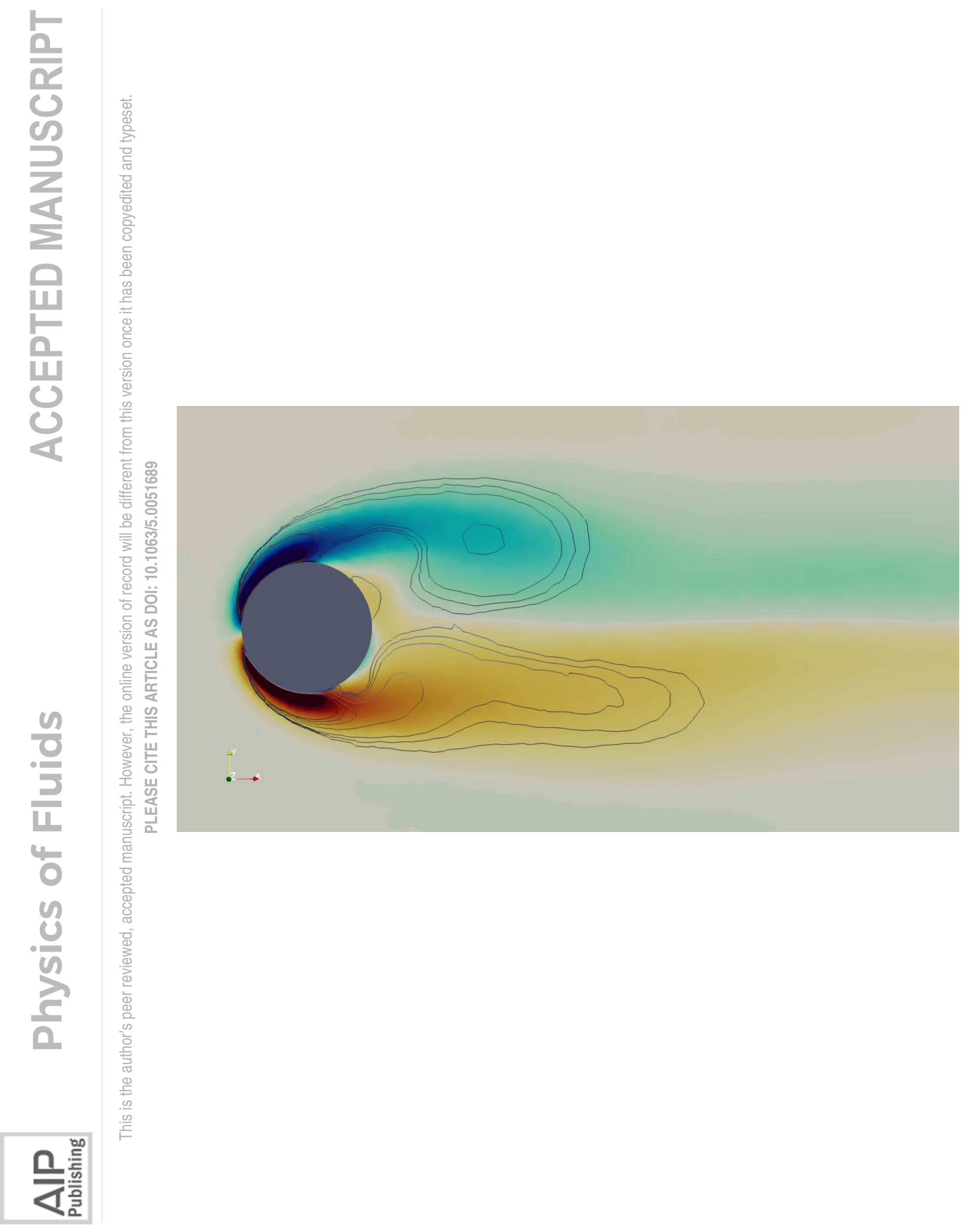




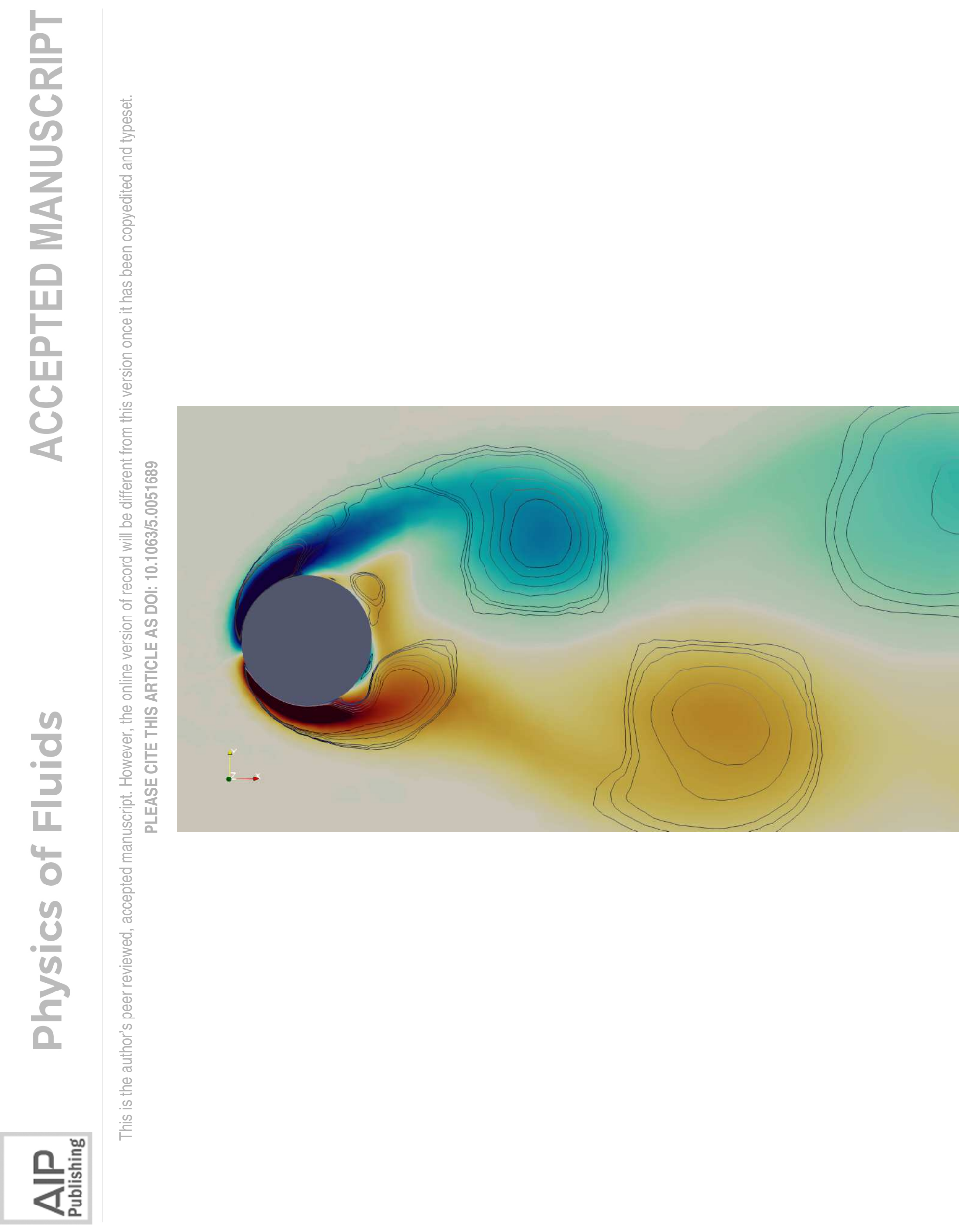




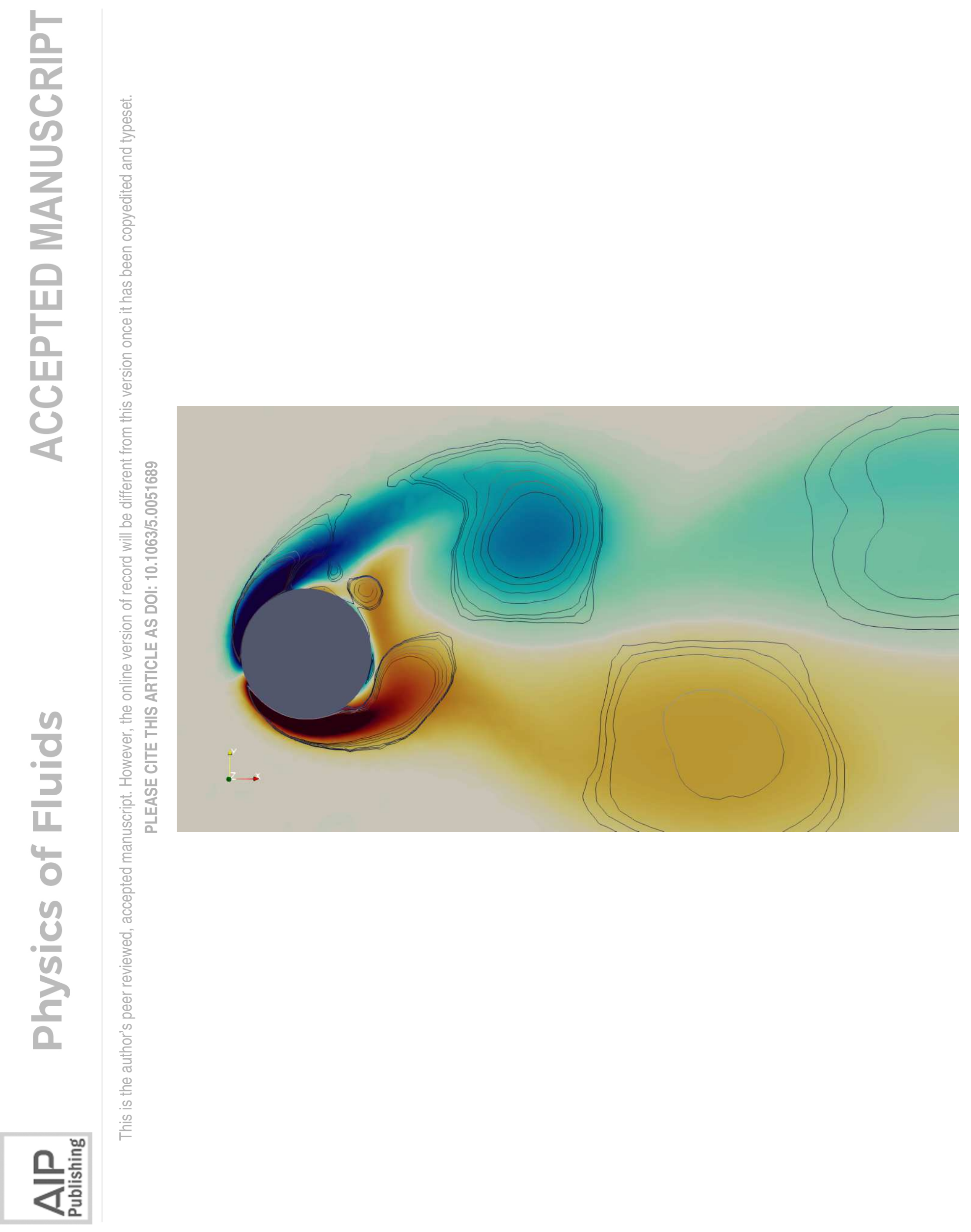




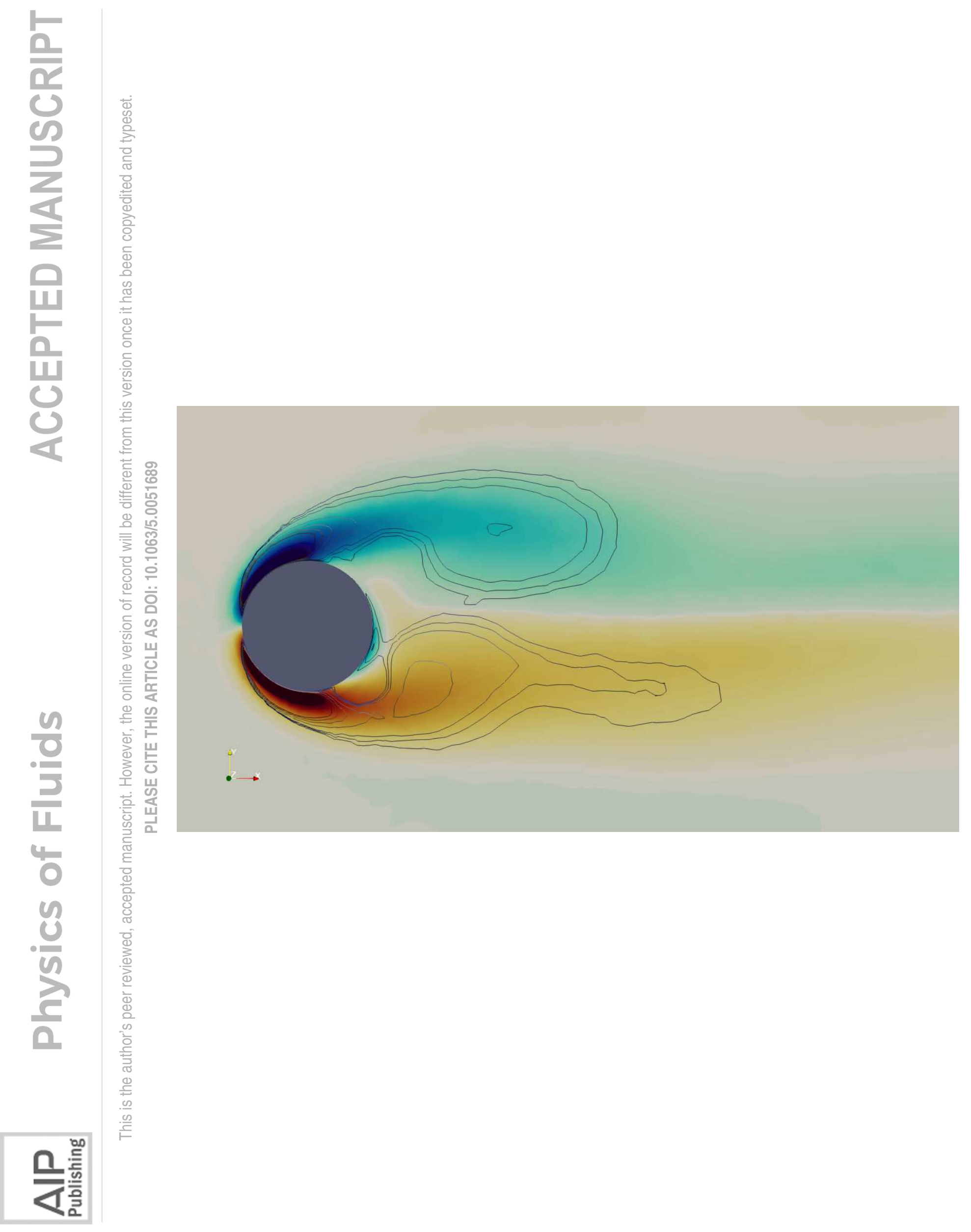




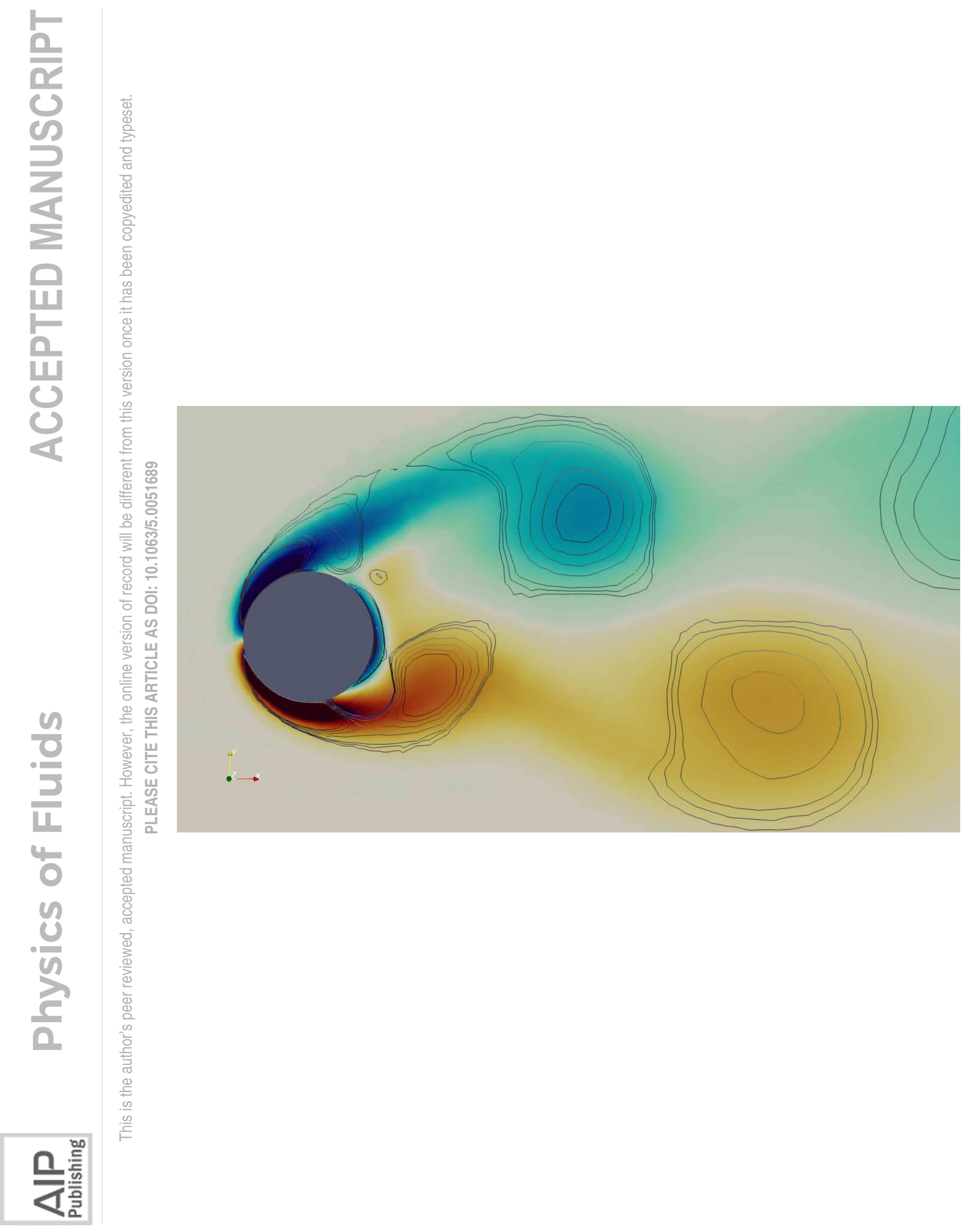




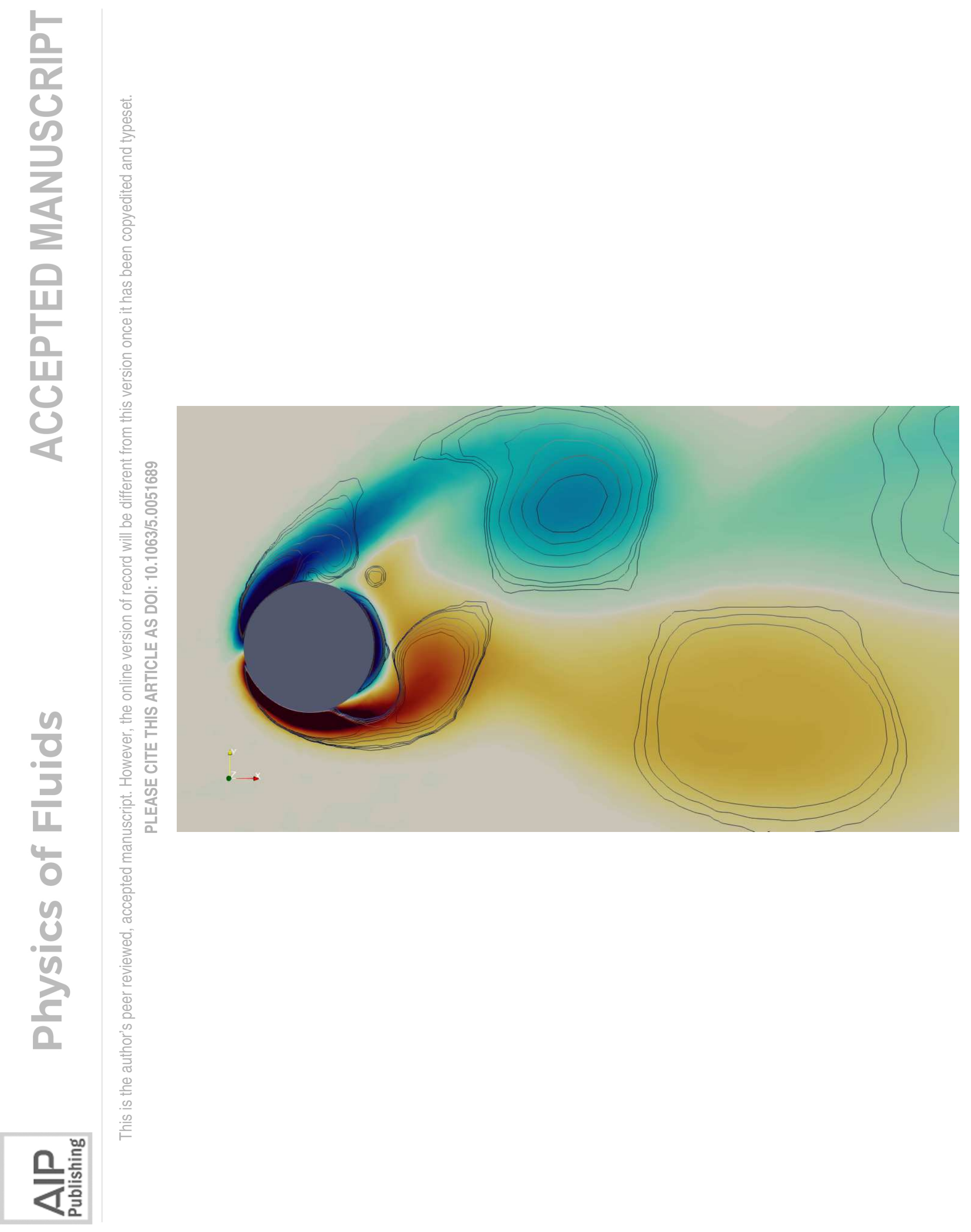




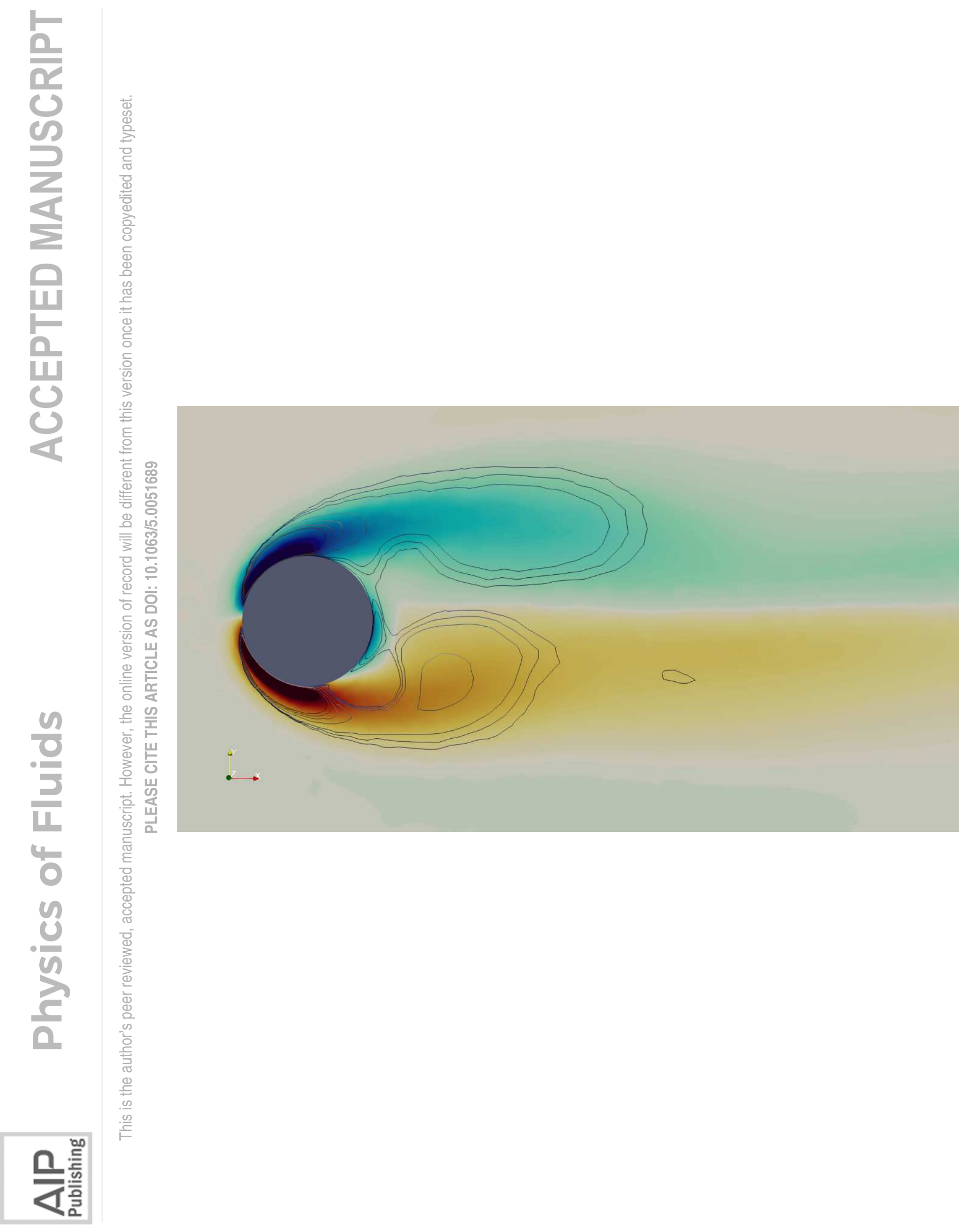




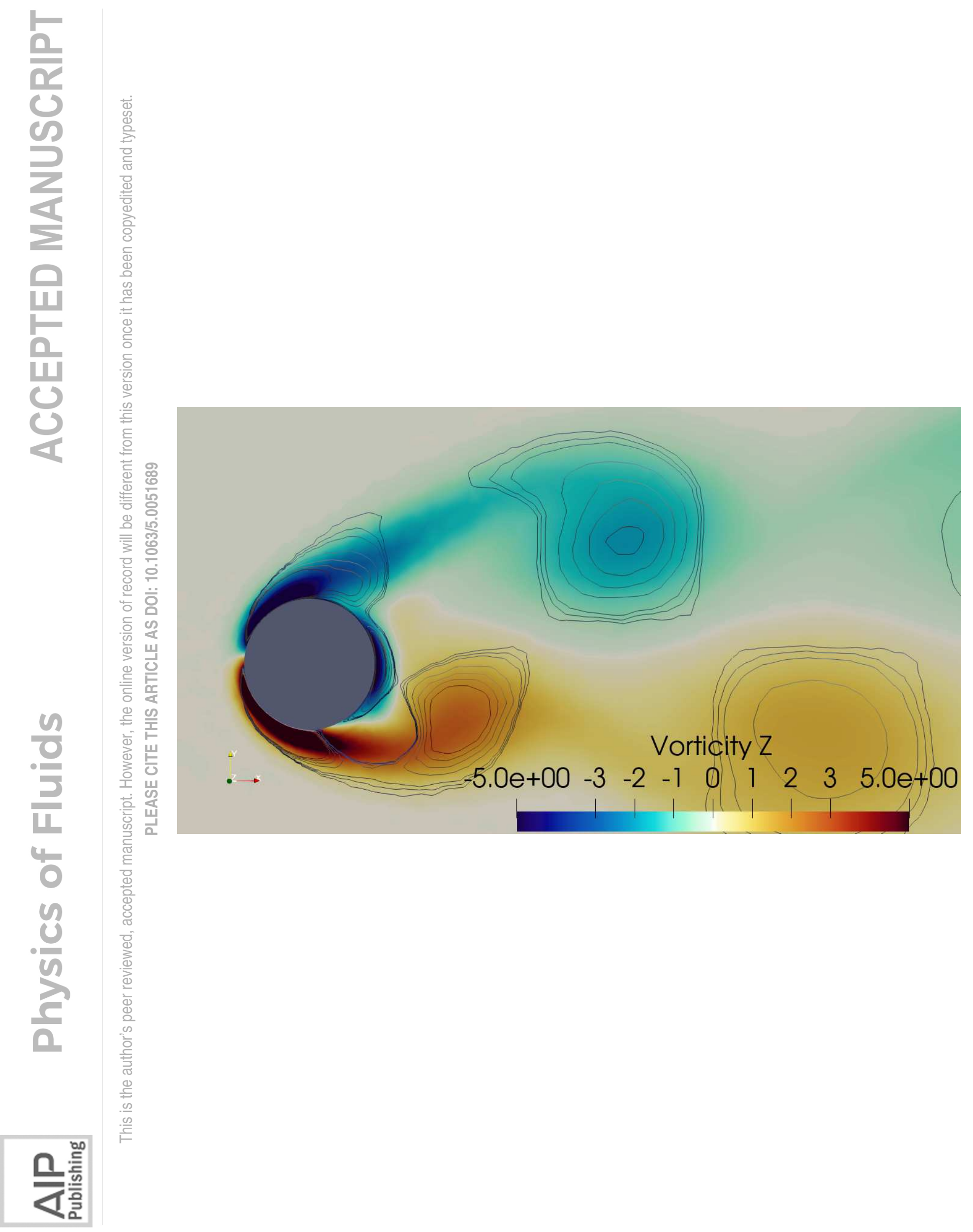




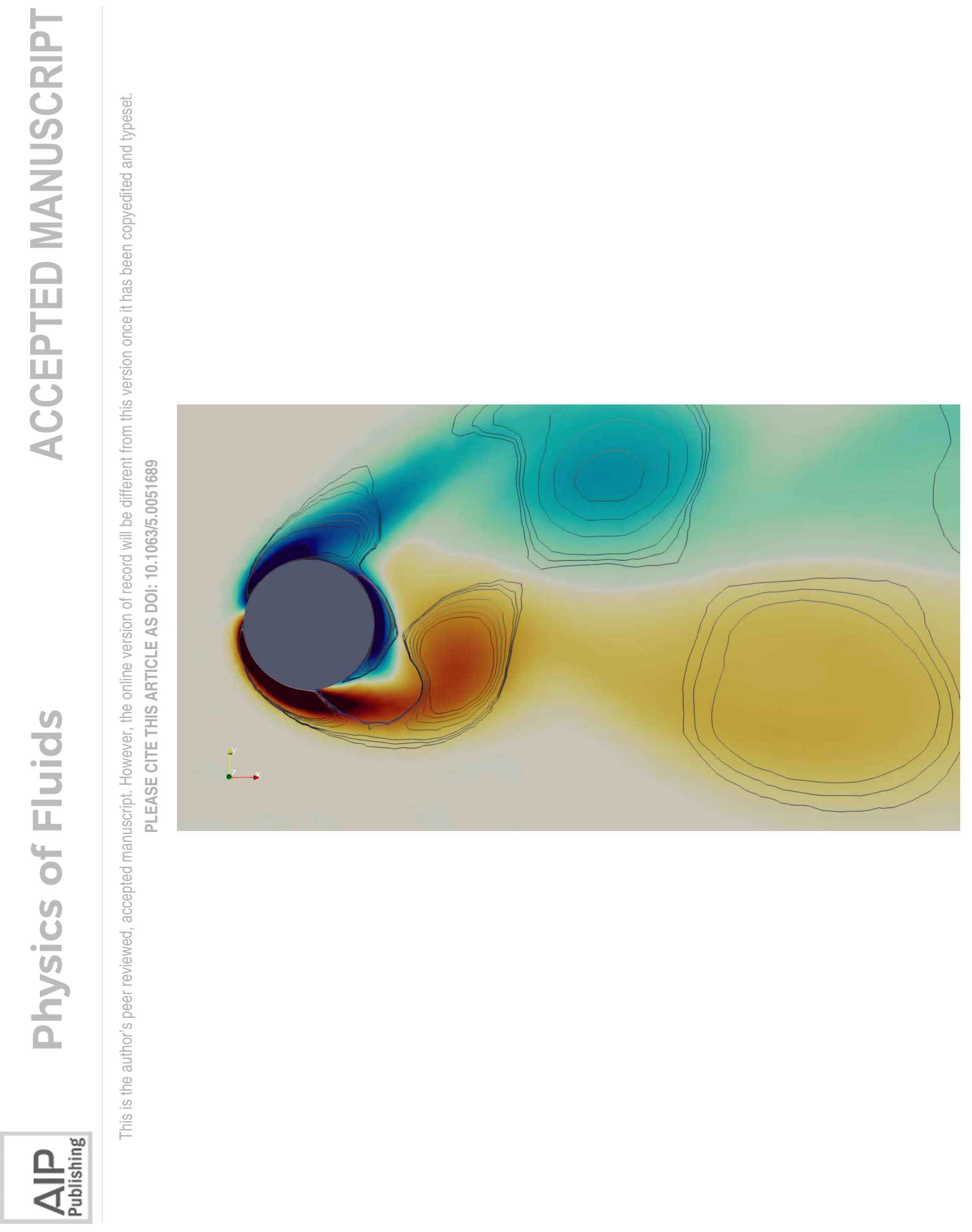




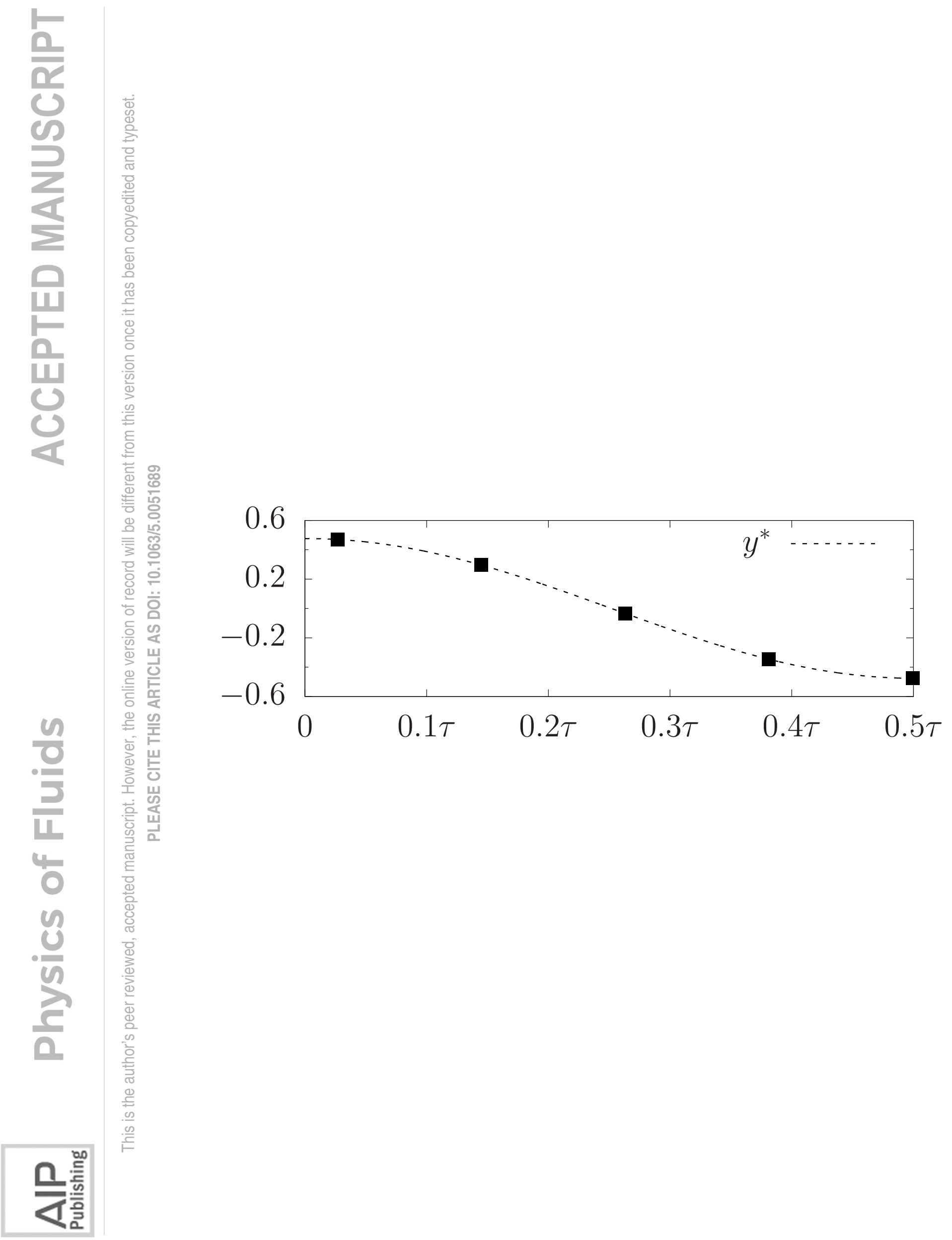




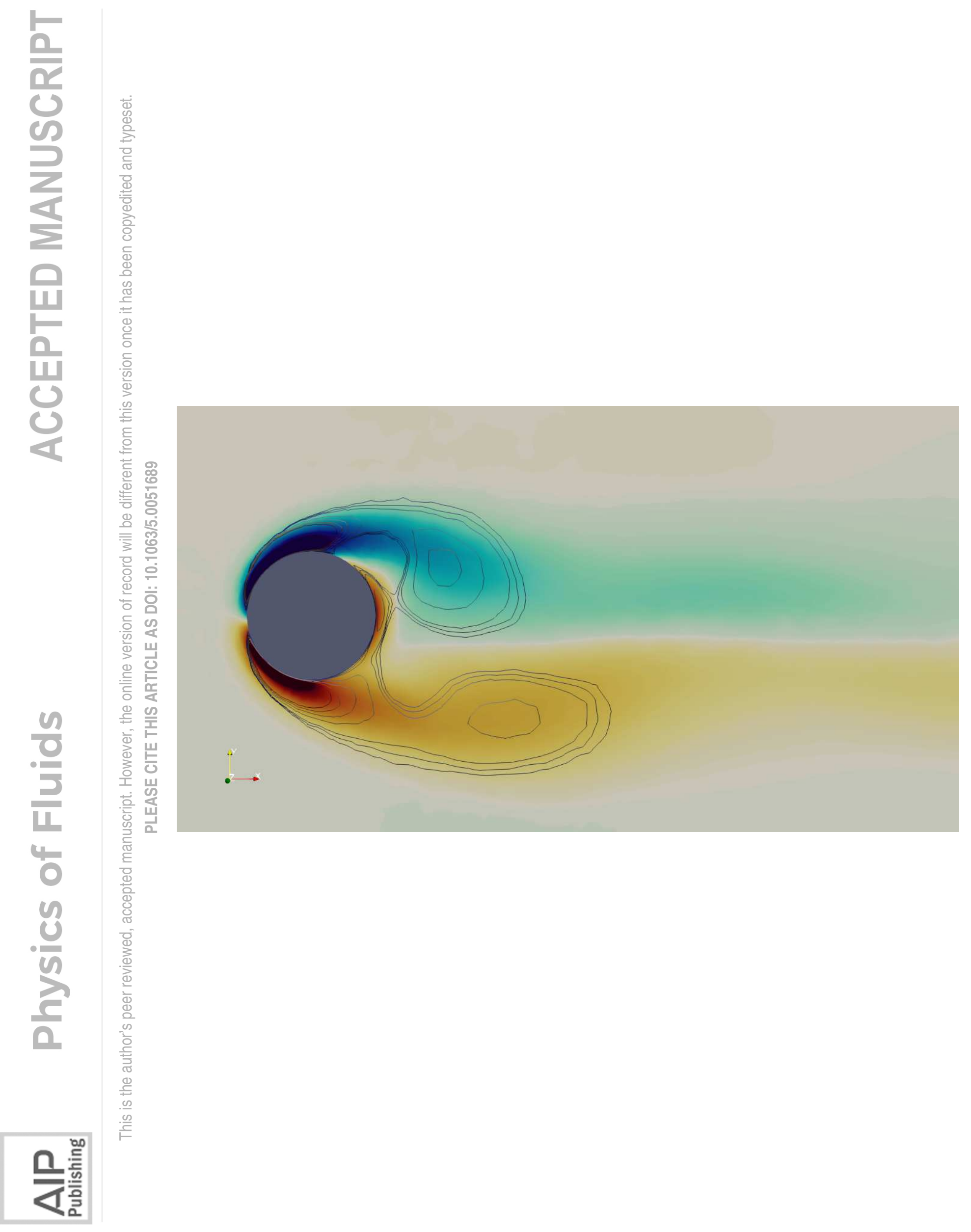




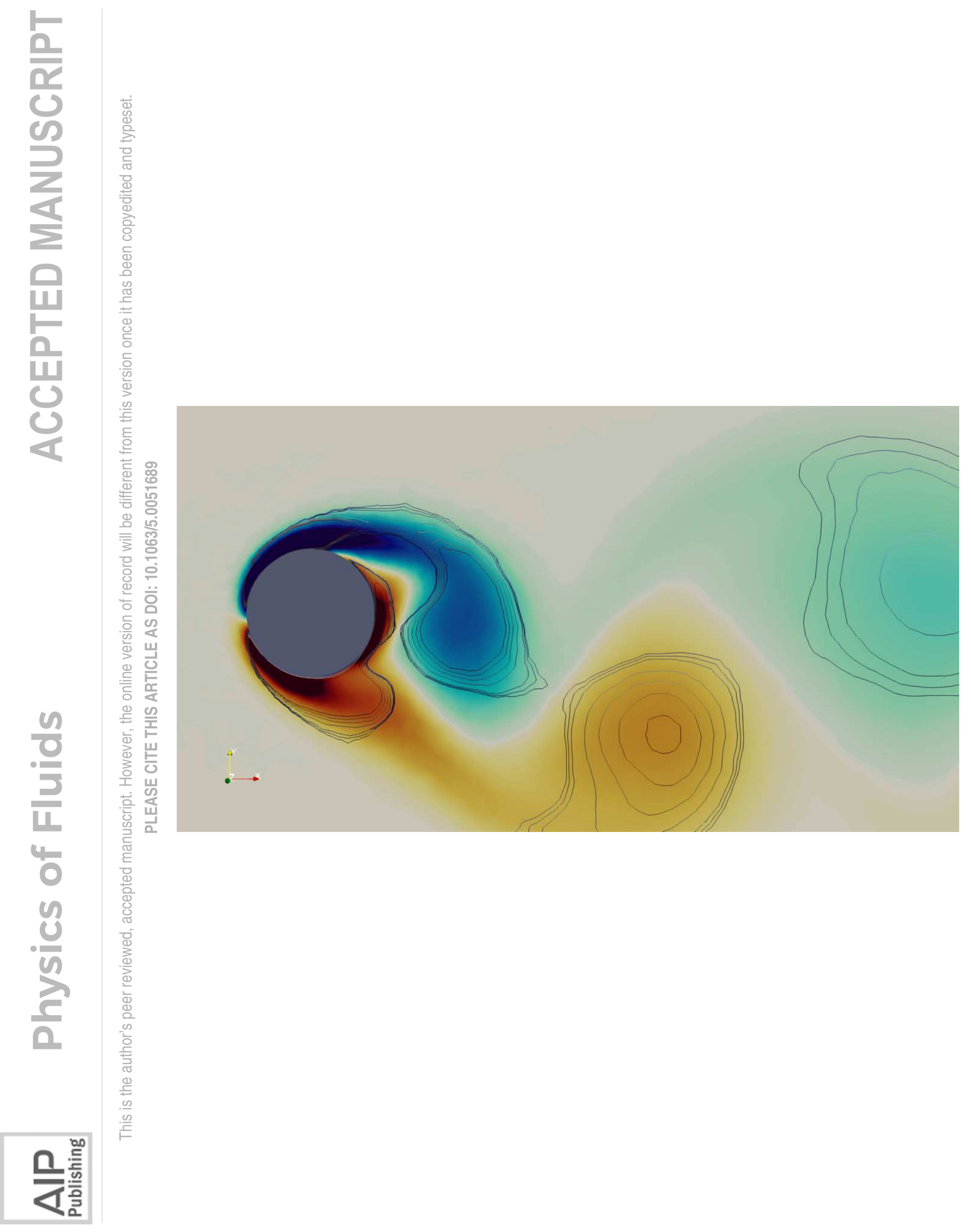




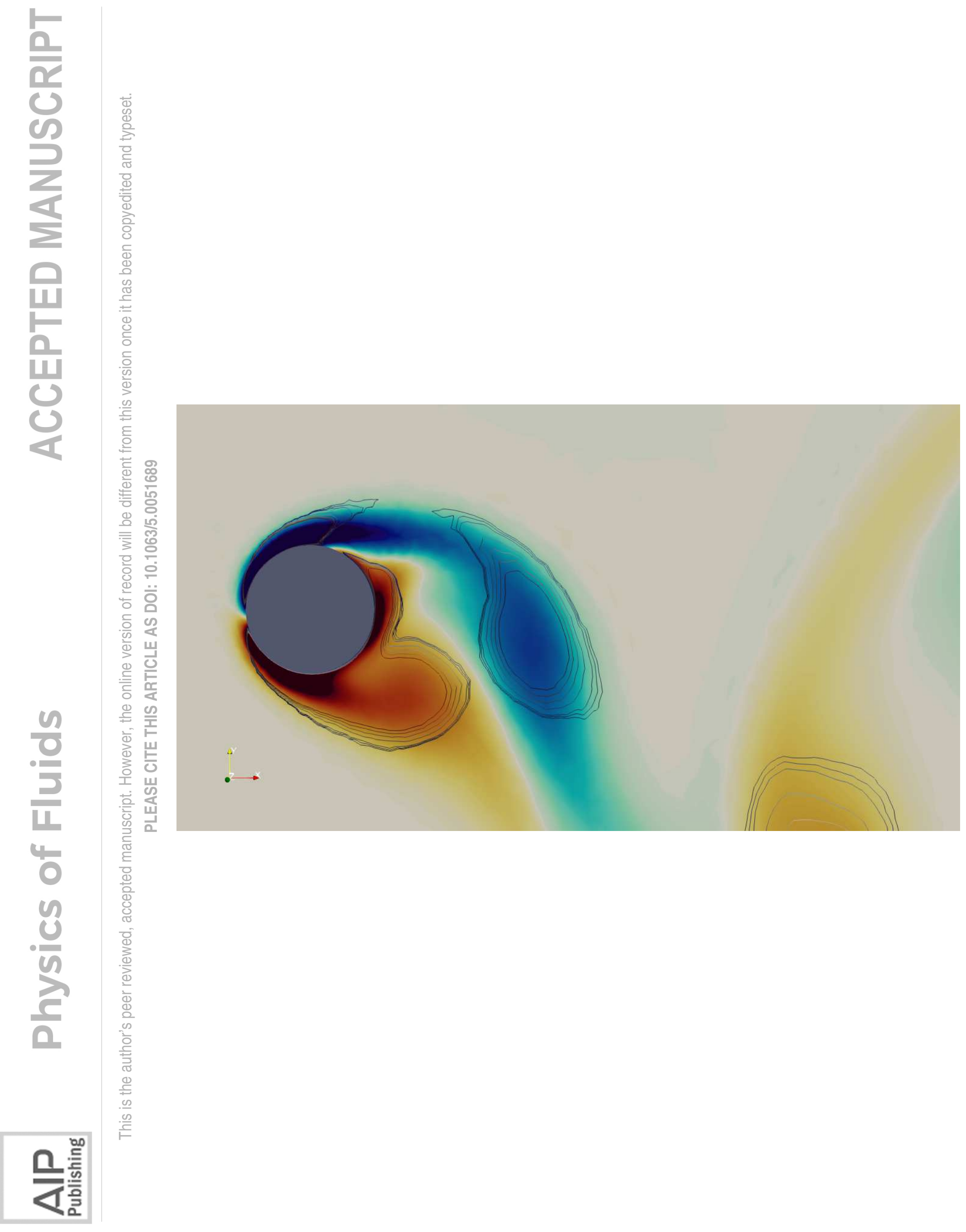




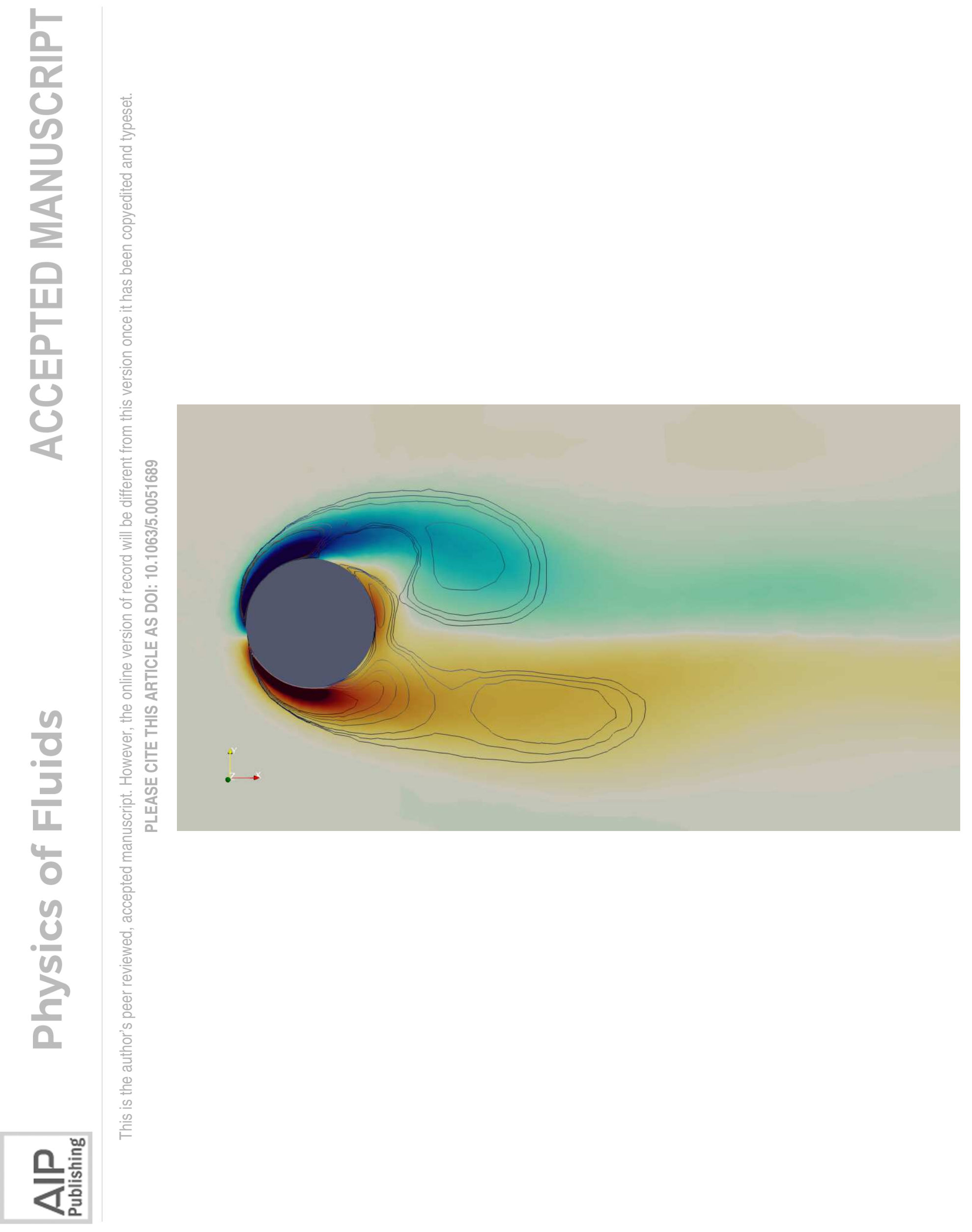




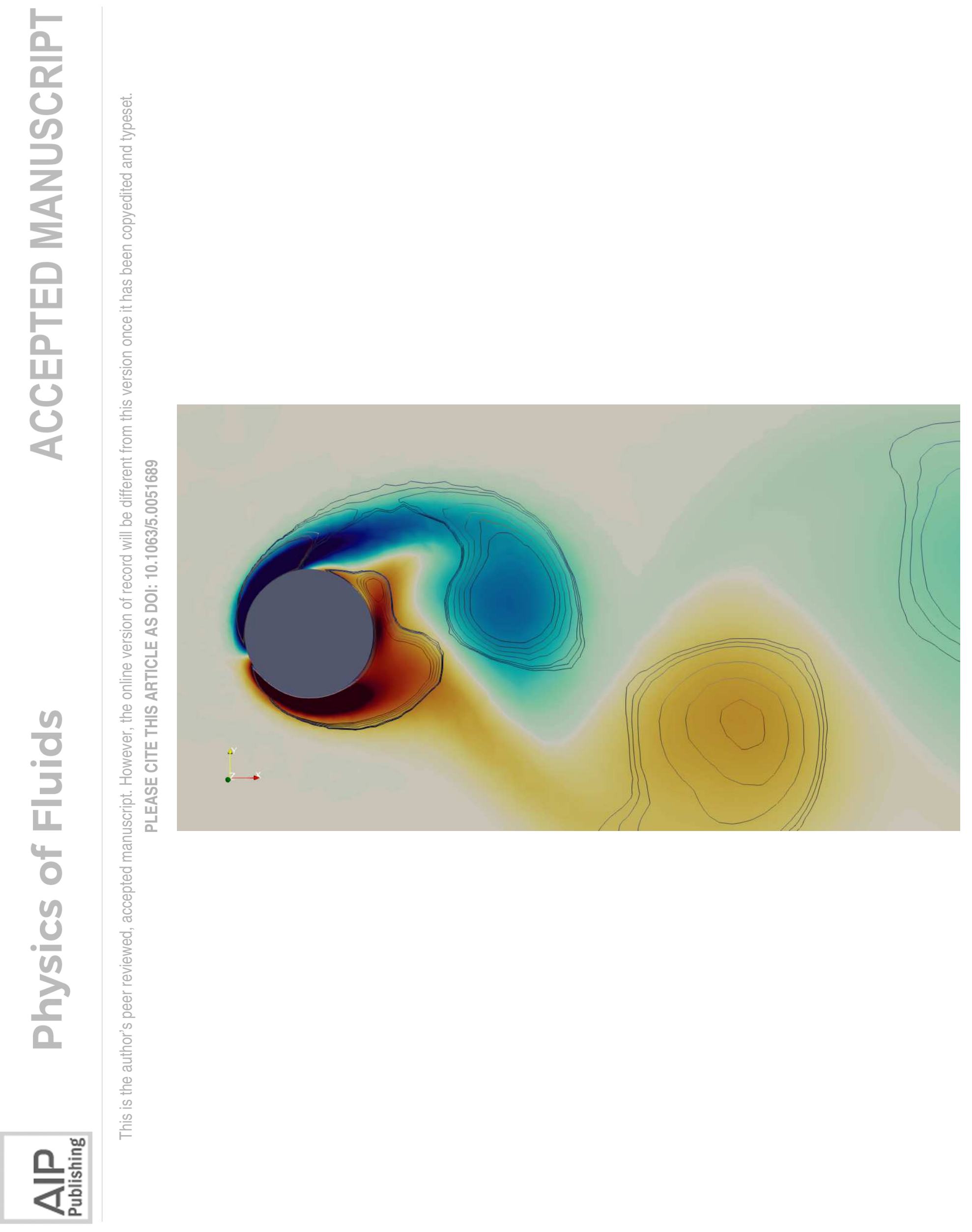




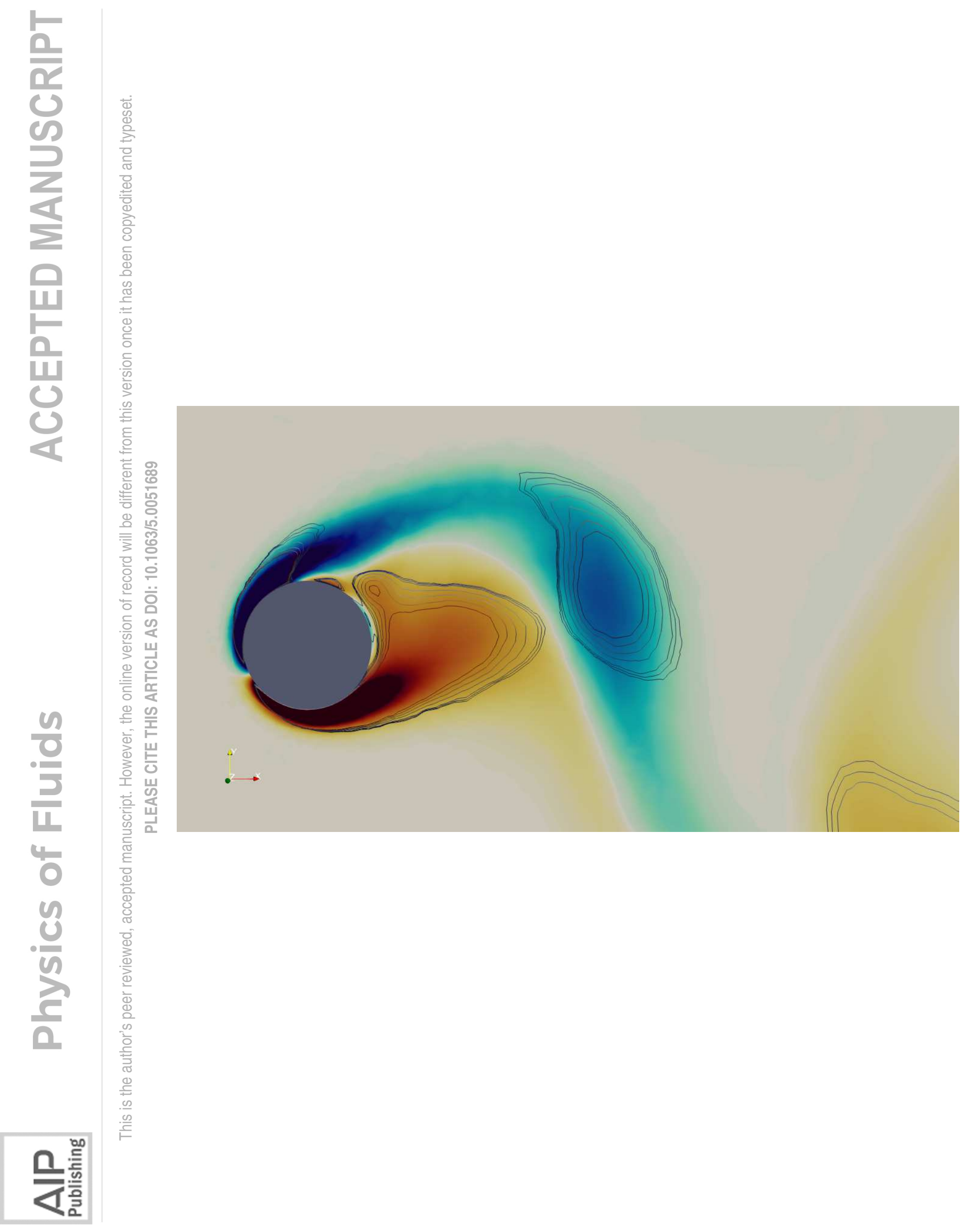




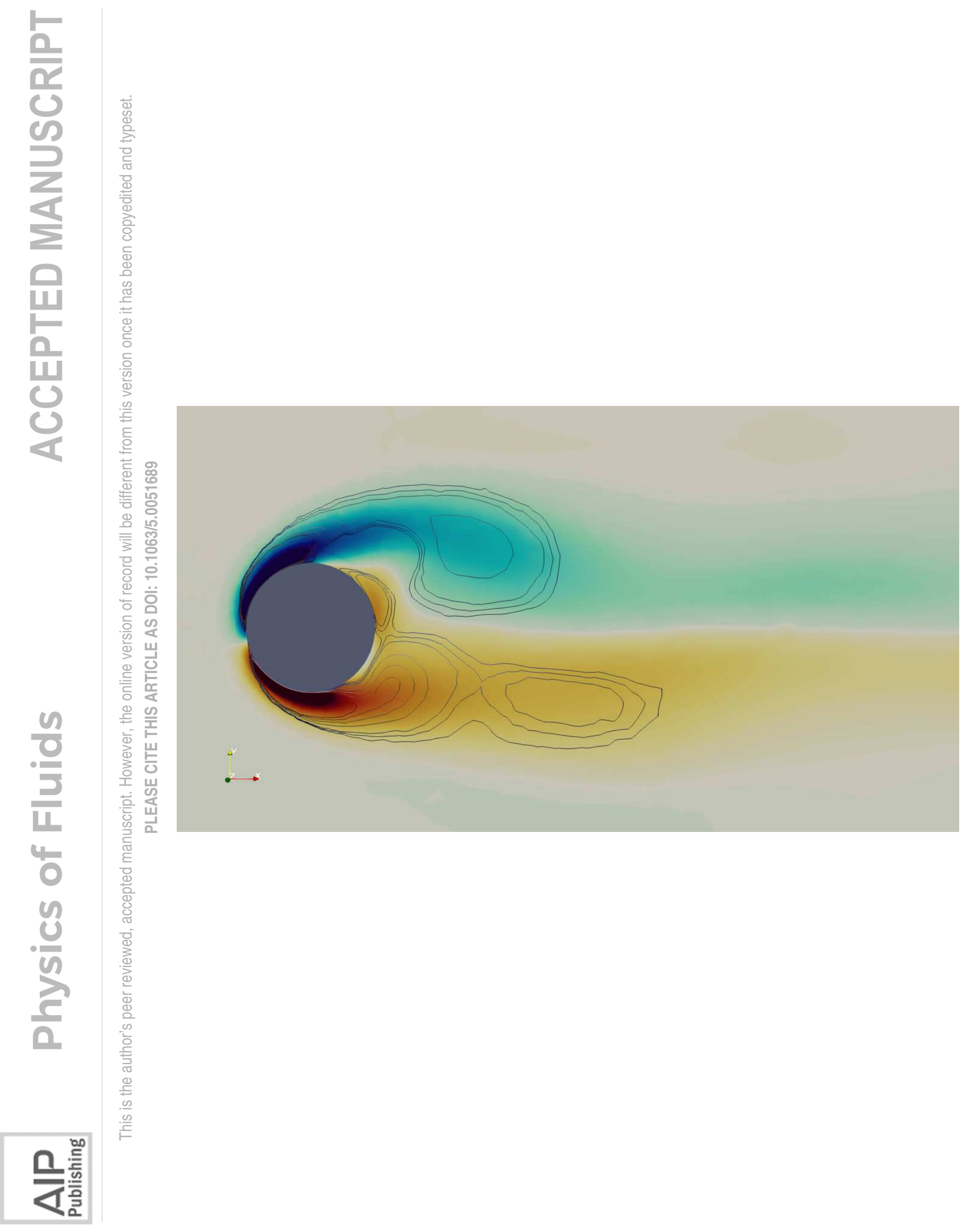




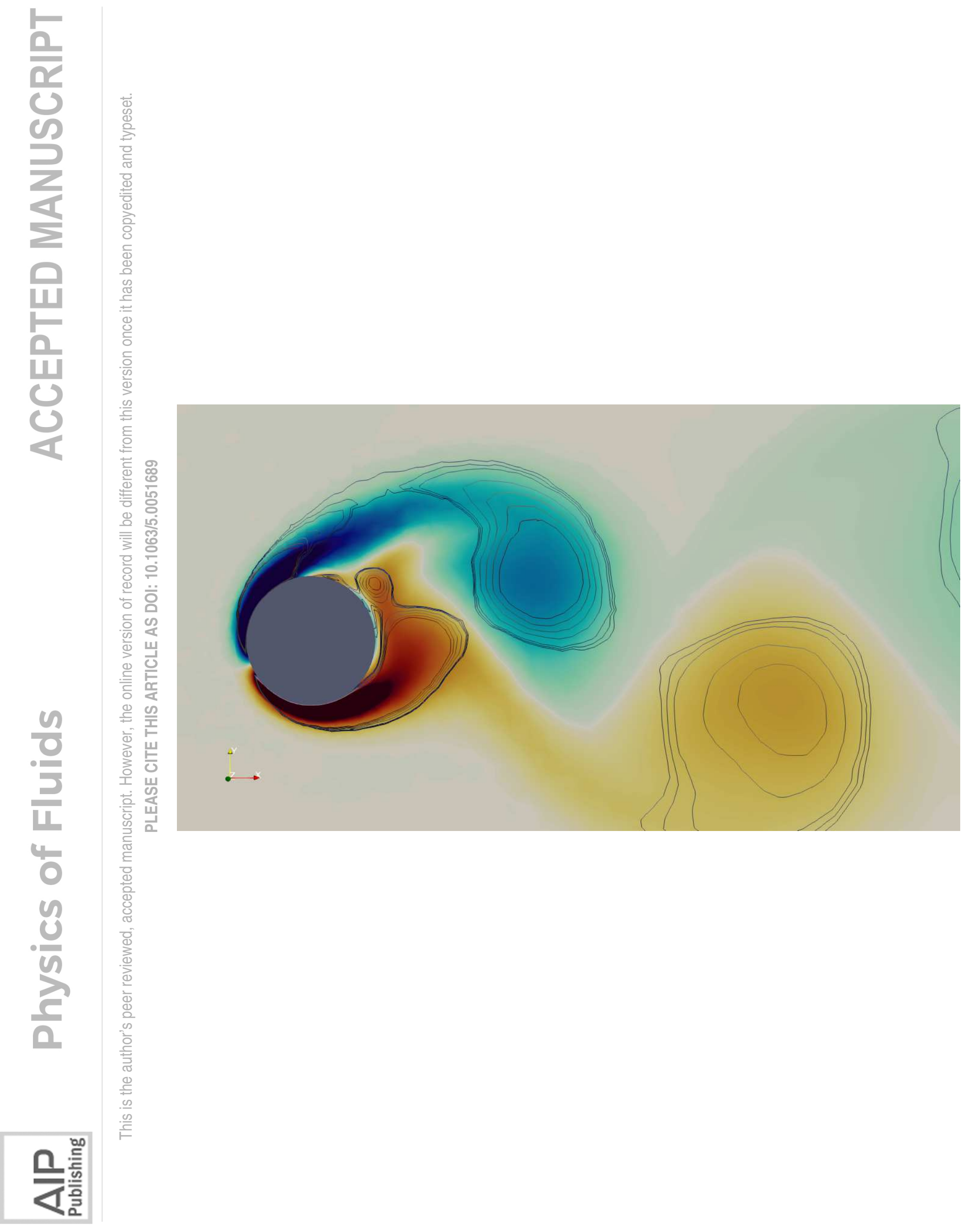




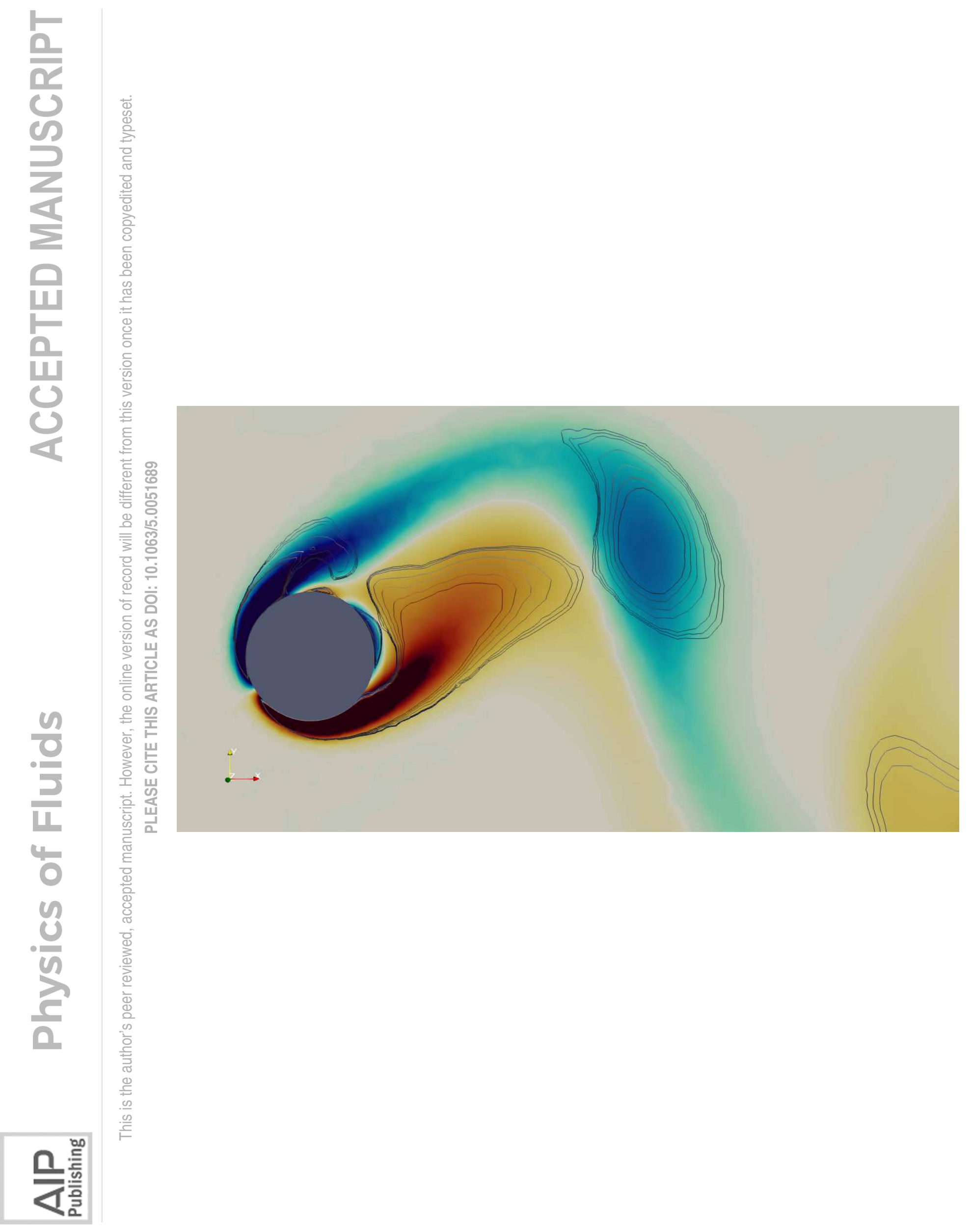




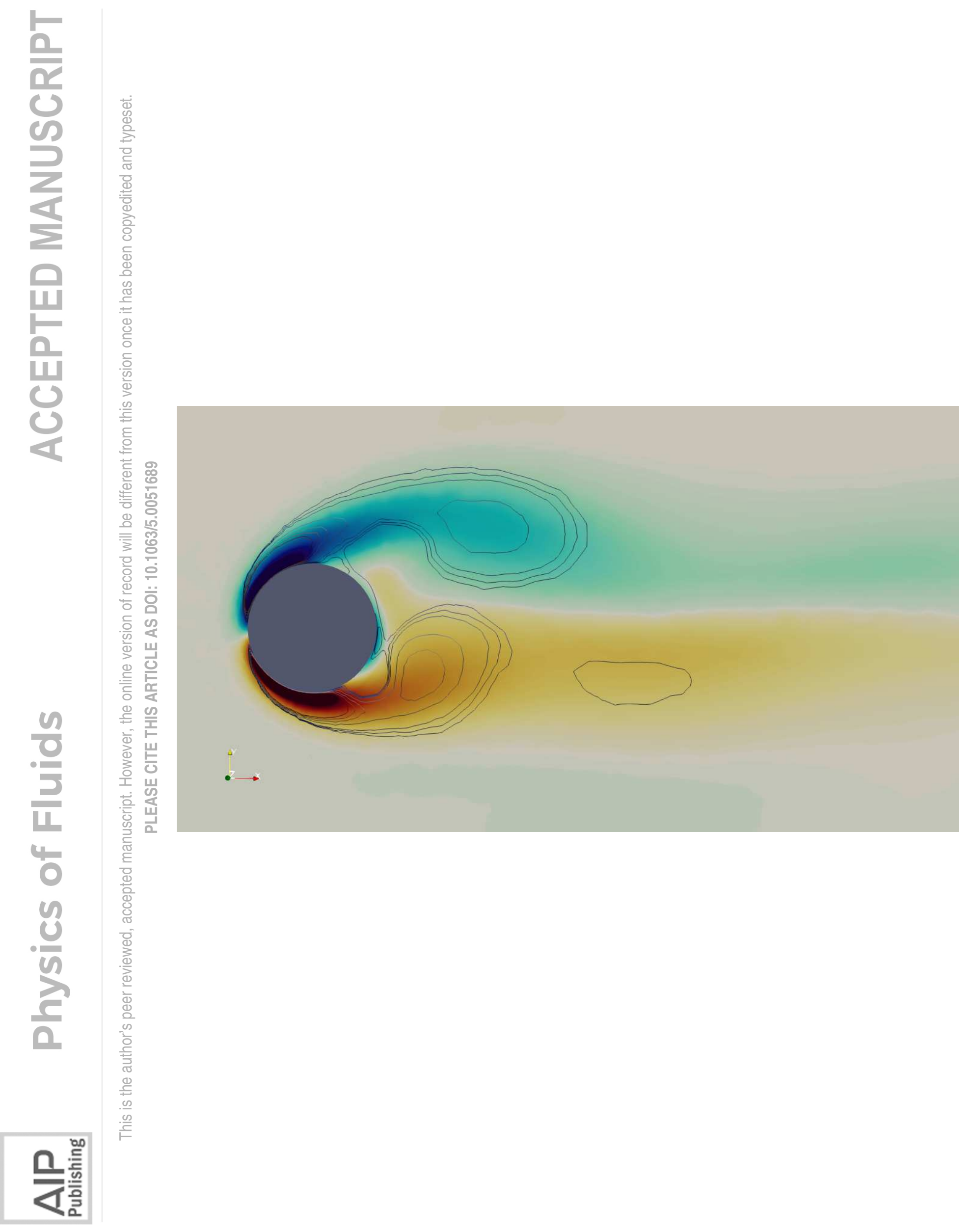




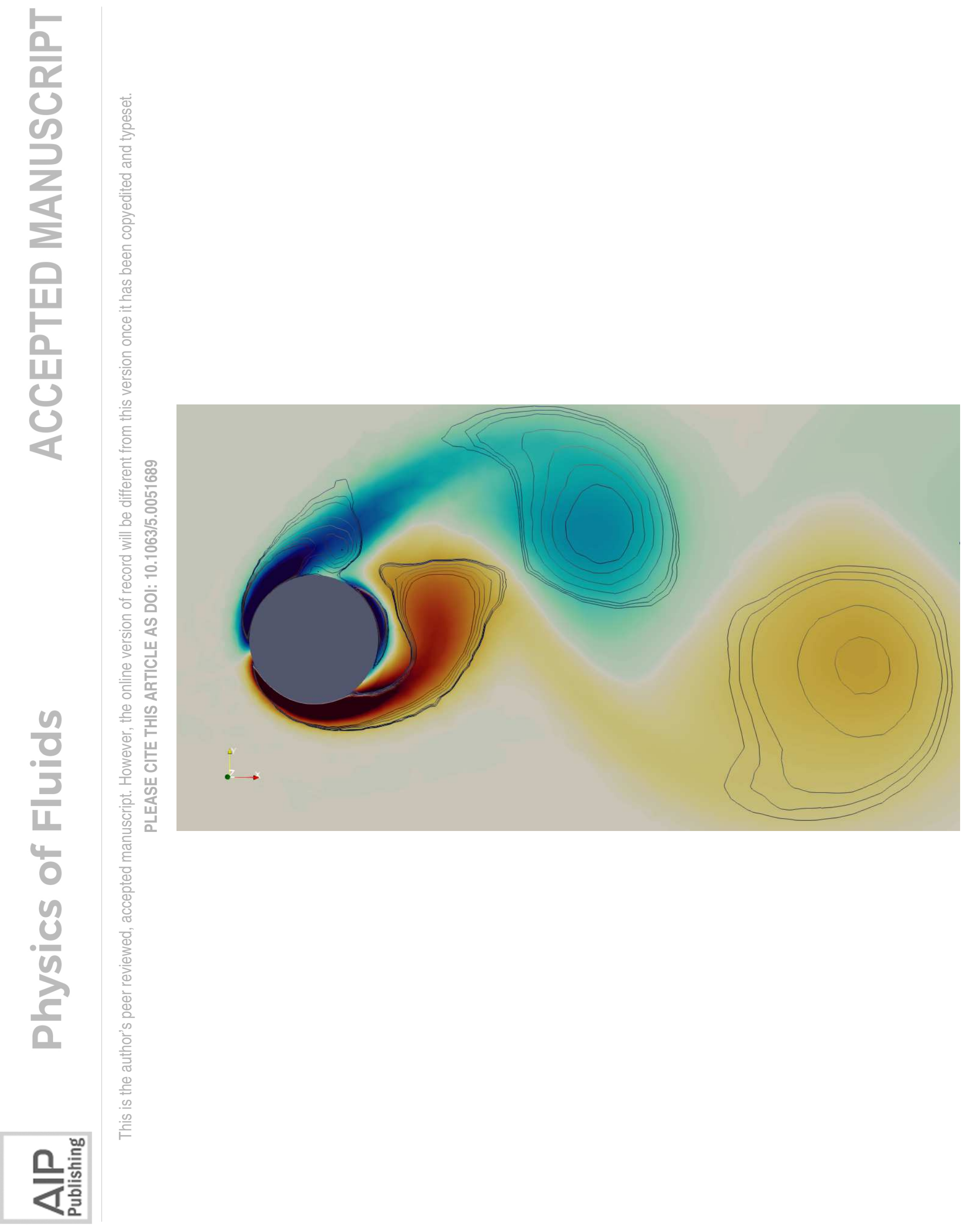




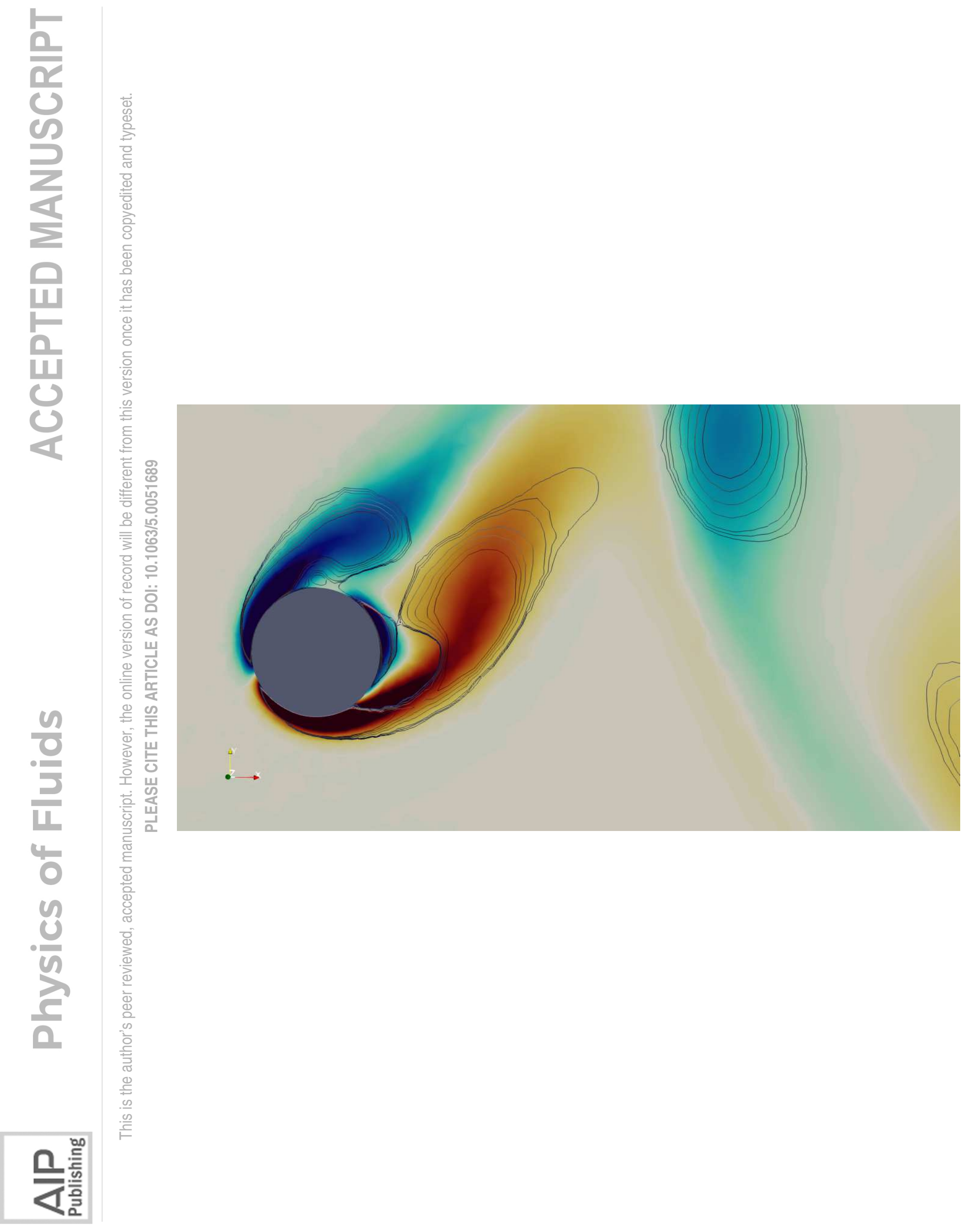




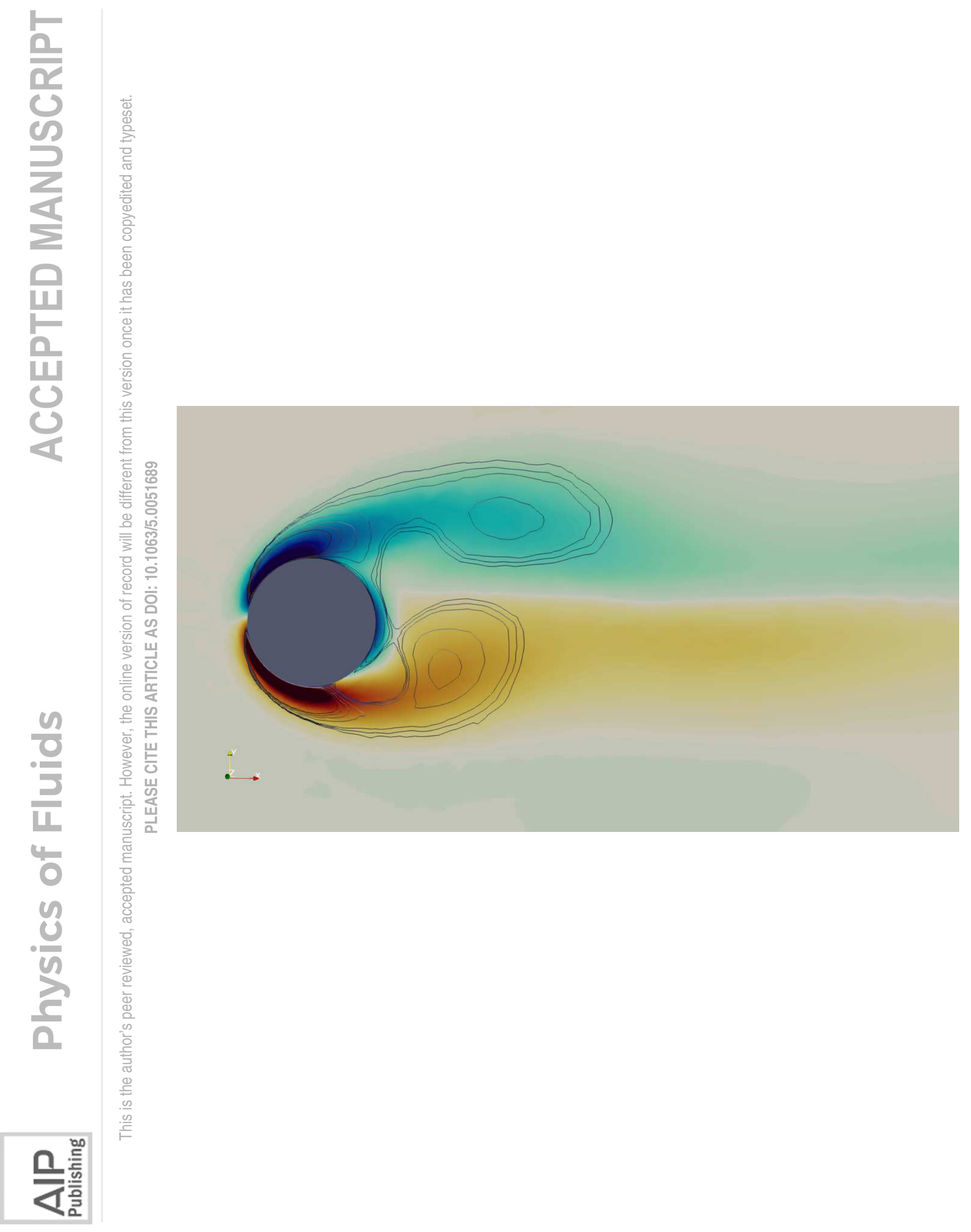




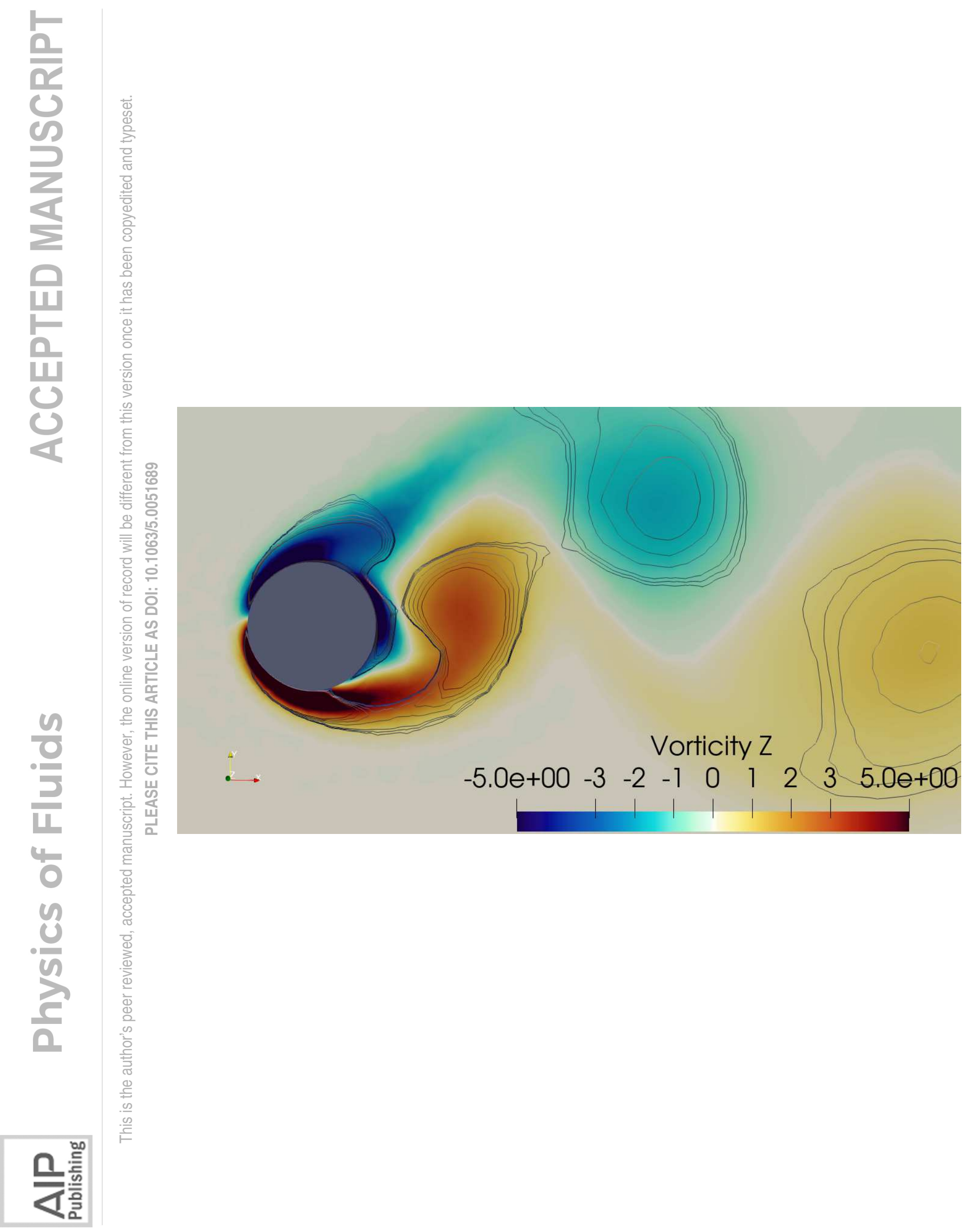




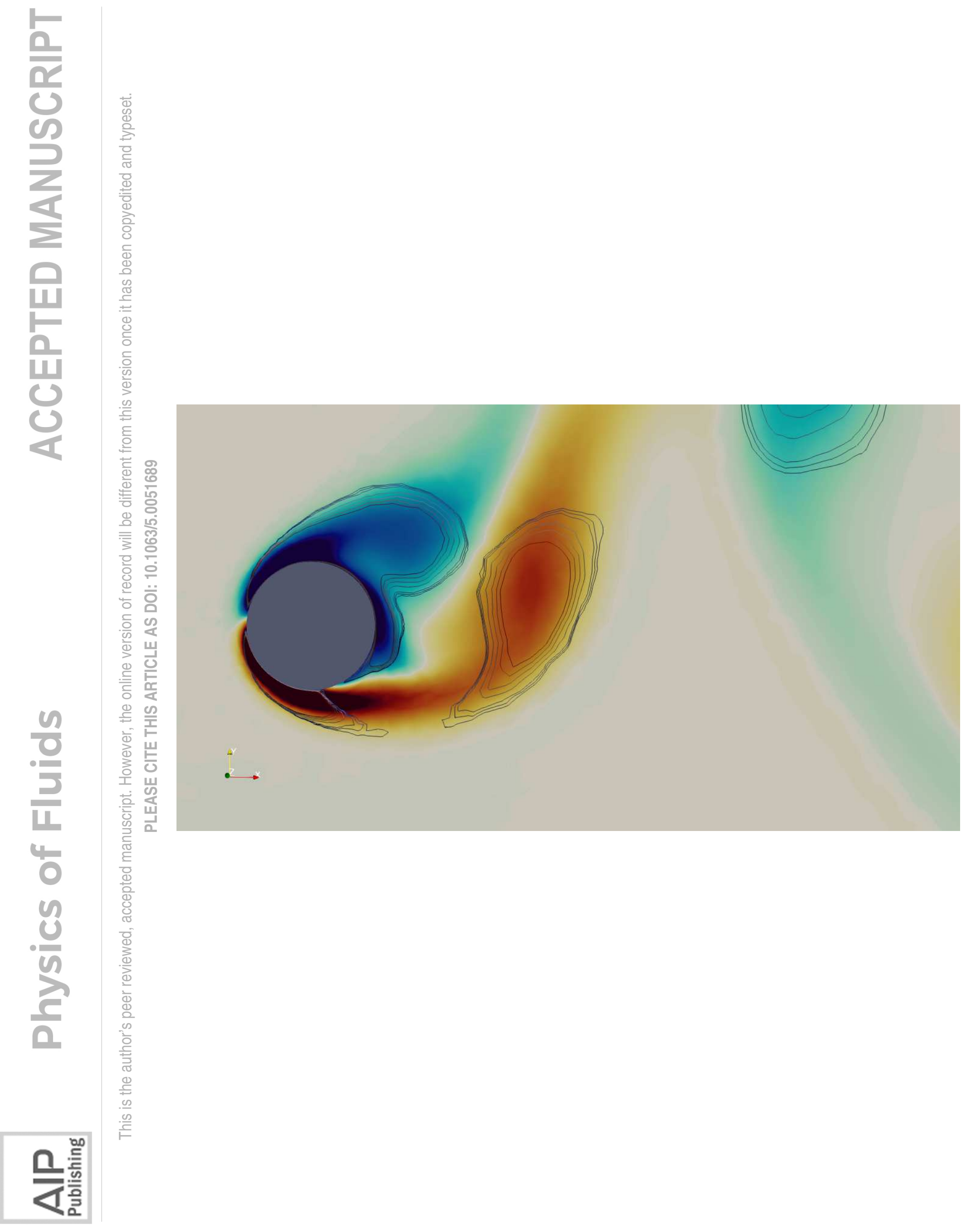




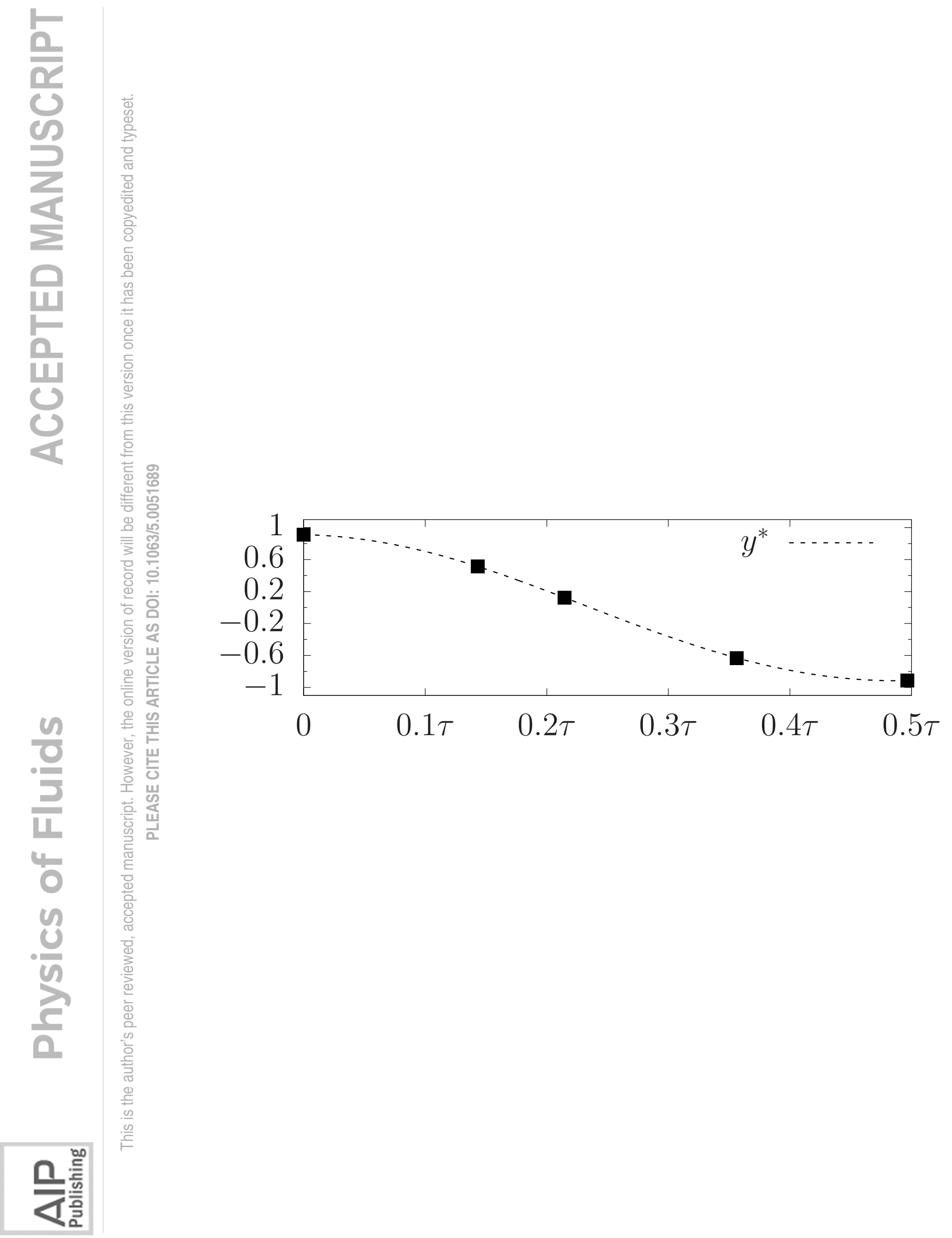




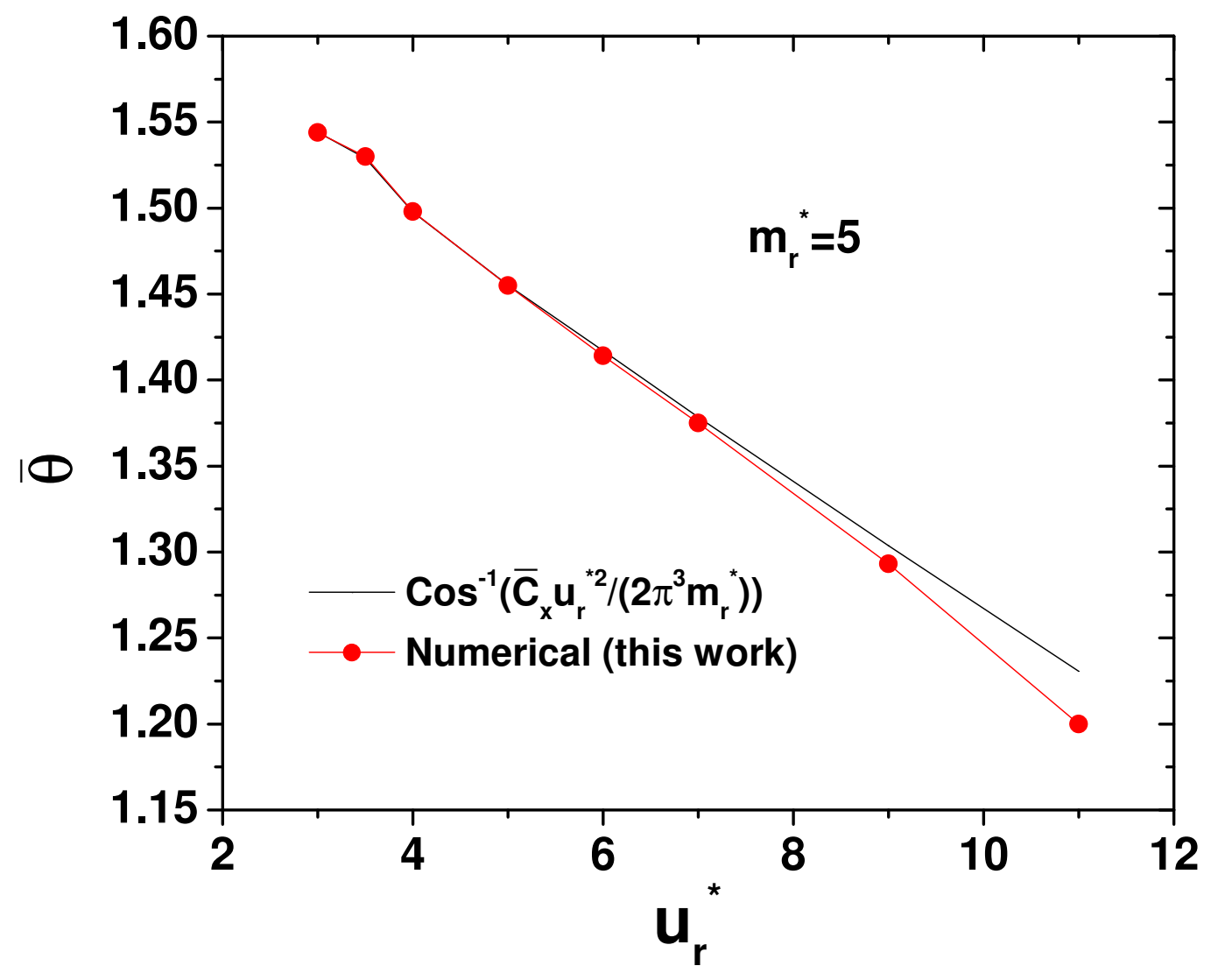




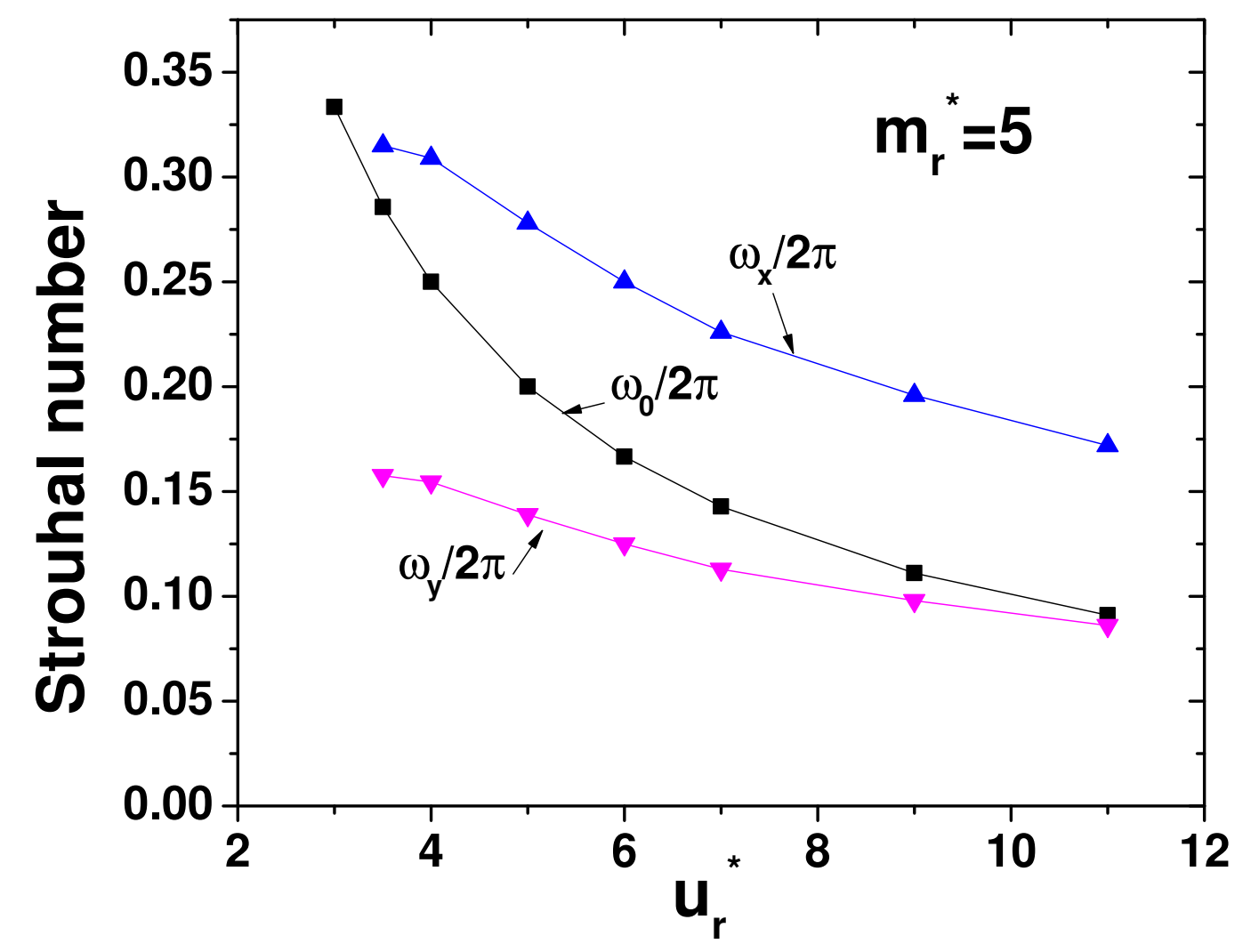

는 


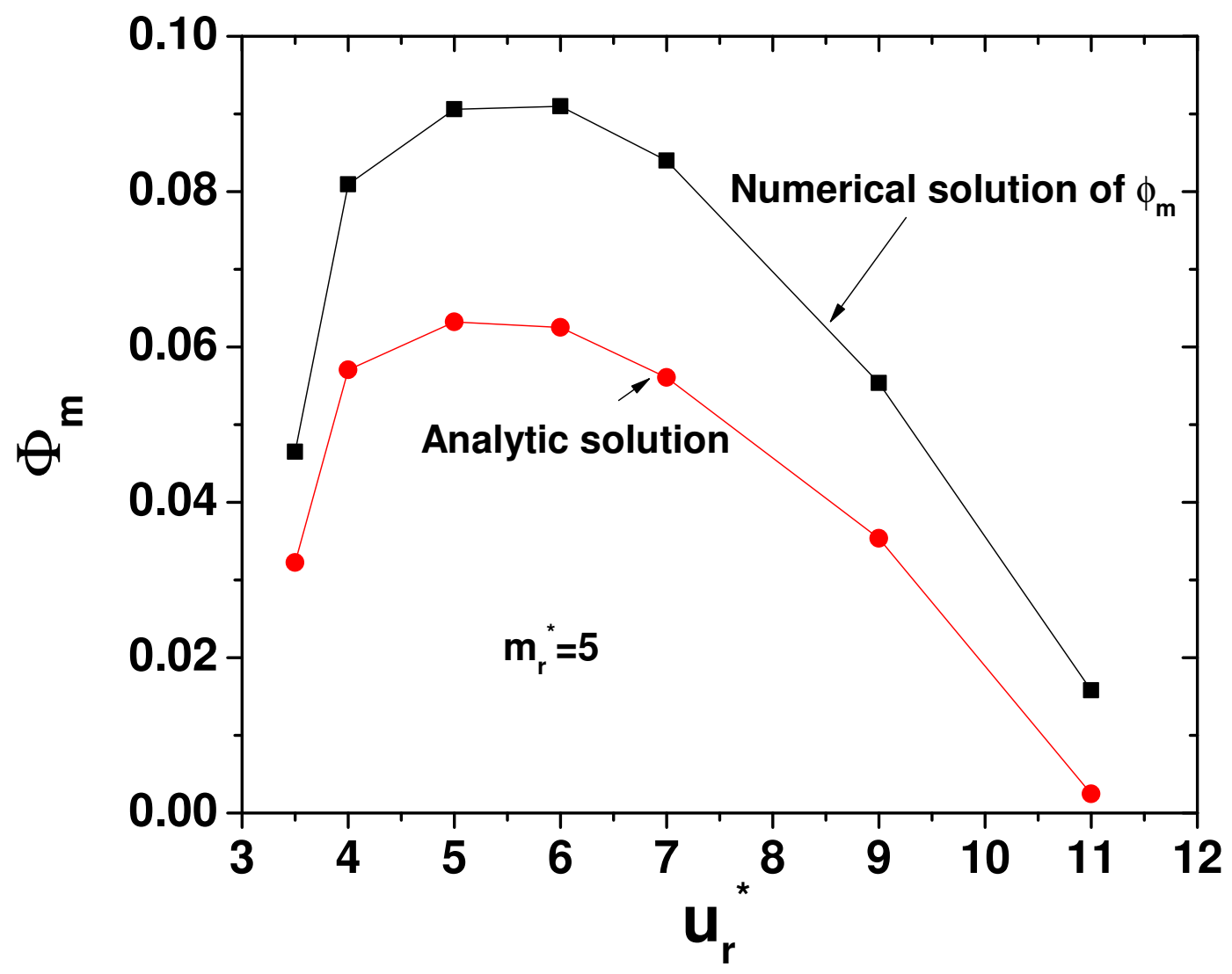

